August 1994

\title{
Dust as a Standard of Space and Time in Canonical Quantum Gravity
}

\author{
J. David Brown \\ Department of Physics and Department of Mathematics, \\ North Carolina State University, Raleigh, NC 27695-8202 \\ Karel V. Kuchar̆ \\ Department of Physics, University of Utah, Salt Lake City, UT 84112
}

\begin{abstract}
The coupling of the metric to an incoherent dust introduces into spacetime a privileged dynamical reference frame and time foliation. The comoving coordinates of the dust particles and the proper time along the dust worldlines become canonical coordinates in the phase space of the system. The Hamiltonian constraint can be resolved with respect to the momentum that is canonically conjugate to the dust time. Imposition of the resolved constraint as an operator restriction on the quantum states yields a functional Schrödinger equation. The ensuing Hamiltonian density has an extraordinary feature: it depends only on the geometric variables, not on the dust coordinates or time. This has three important consequences. First, the functional Schrödinger equation can be solved by separating the dust time from the geometric variables. Second, the Hamiltonian densities strongly commute and therefore can be simultaneously defined by spectral analysis. Third, the standard constraint system of vacuum gravity is cast into a form in which it generates a true Lie algebra. The particles of dust introduce into space a privileged system of coordinates that allows the supermomentum constraint to be solved explicitly. The Schrödinger equation yields a conserved inner product that can be written in terms of either the instantaneous state functionals or the solutions of constraints. Gravitational observables admit a similar dual representation. Examples of observables are given, though neither the intrinsic metric nor the extrinsic curvature are observables. Disregarding the standard factor-ordering difficulties, the introduction of dust provides a satisfactory phenomenological approach to the problem of time in canonical quantum gravity.
\end{abstract}

Typeset using REVTEX 


\section{INTRODUCTION}

The Dirac constraint quantization of vacuum Einstein gravity yields the Wheeler-DeWitt equation for the quantum state of the intrinsic three geometry of space [1,2]. One can view this equation as a statement that only two out of three independent components of the intrinsic geometry are dynamical. The third component is an intrinsic time that specifies the location of space as a hypersurface in spacetime. The Wheeler-DeWitt equation is then interpreted as an evolution equation for the state in the intrinsic time.

The Wheeler-DeWitt equation is a second-order variational differential equation. The space of its solutions carries no obvious Hilbert space structure [3,, t. This has prompted numerous attempts aimed at replacing the Wheeler-DeWitt equation by a first-order Schrödinger equation. In order to do that, one should identify the intrinsic time at the classical level, solve the Hamiltonian constraint for the momentum conjugate to time, and impose the resolved constraint as an operator restriction on the quantum states. Unfortunately, there is no natural candidate for the intrinsic time, and the procedure is beset by a number of conceptual and technical difficulties [0].

Intrinsic clocks are strange contraptions. From the early days of general relativity, most researchers felt that spacetime intervals are to be measured not by internal clocks but by material devices, analogous to the measurement of electromagnetic fields by test charges. At the most basic level, matter splits the spacetime manifold into space and time. The phenomenological medium introduced for this purpose is the reference fluid [6]. The particles of the reference fluid identify the points of space, and clocks carried by these particles identify the instants of time. In this way, the fluid fixes the reference frame (the space) and the time foliation (the time). In that frame and on that foliation, the entire intrinsic metric, not just two selected components of the intrinsic geometry, becomes dynamical.

The reference fluid is traditionally considered to be a tenuous material medium whose back reaction on the geometry can be neglected. There are just enough fluid particles to discern the points of space from one another, but not enough to disturb the geometry. Instead of deriving the motion of the fluid from its action, one encodes it in coordinate conditions. Unfortunately, such a standpoint makes it difficult to view the reference fluid as physical matter.

There are two alternative routes through which the reference fluid can be turned into a physical system. The first is to impose the coordinate conditions before variation by adjoining them to the Hilbert action with Lagrange multipliers [10,11]. Equivalently, a Lagrangian might be devised to enforce these conditions [12, 13]. The additional terms in the action can be interpreted as a matter source coupled to the gravitational field. A disadvantage of this approach is that simple coordinate conditions often lead to contrived matter sources. The second route is to select a realistic material medium for the fluid and to describe its properties by a physical Lagrangian [14,2]. Again, by adding this Lagrangian to the Hilbert Lagrangian, the fluid becomes coupled to gravity. The challenge is to find a medium whose space and time variables can be turned into canonical coordinates that lead, upon the Dirac constraint quantization, to a simple Schrödinger equation for canonical quantum gravity.

Our goal is to show that the most simple type of matter conceivable, incoherent dust, is just what one needs to obtain a satisfactory framework for interpreting quantum gravity. 
The particles of dust follow timelike geodesics, and in this sense dust is quite special: Dust is the 'purest' form of matter, matter that interacts with itself only via the gravitational field.

The canonical scheme offered by incoherent dust has an extraordinary feature: The many-fingered Hamiltonian of the ensuing functional Schrödinger equation does not depend on the dust variables. This fact has a number of important consequences. First, the Hamiltonian densities strongly commute (modulo factor-ordering difficulties), and thus can be simultaneously defined by spectral analysis. Second, the functional Schrödinger equation can be solved by separating the dust time from the gravitational variables. Third, the fact that the Hamiltonian depends only on the gravitational variables leads to the discovery of a form for the constraint system of vacuum gravity in which the constraints generate a true Lie algebra.

The dust particles also endow space with a privileged, physical system of coordinates. Canonical variables such as the spatial metric can be specified as tensors in these dust coordinates, and in this way the combined system of gravity and dust can be expressed in a form that does not rely on arbitrary systems of spatial coordinates [15]. As a consequence, the supermomentum constraint, which is the canonical generator of spatial diffeomorphisms, becomes trivial and is exactly solvable in the quantum theory.

All of the above features combine into an intriguing scheme for interpreting canonical quantum gravity. Although we focus on the case of gravity coupled only to dust, the scheme can be applied in the presence of other matter couplings as well — as long as dust is included in the list of matter fields, all of our basic conclusions remain intact. Here is how the scheme works:

We design a spacetime action whose variation yields the Euler equations of motion for an incoherent dust (Section 2a). All of the state variables have a direct physical significance. In particular, four of these variables are the proper time and the dust frame (Section $2 \mathrm{~b}$ ); these are the desired tools for interpreting quantum gravity. The action is unchanged by a subgroup of diffeomorphisms in the dust spacetime manifold (Appendix C). These global symmetries yield an infinite set of Noether charges (Section 2e) that are conserved by virtue of continuity equations (Section 2c). By varying the dust action with respect to the spacetime metric, the standard energy-momentum tensor is obtained (Section 2d). The dust action can be cast into several equivalent forms (Appendix B).

The ADM decomposition of the dust action, followed by a Legendre dual transformation, yields the canonical description of dust (Section 3a). The canonical coordinates coincide with the dust time and frame variables, while the conjugate momenta are simply related to the mass density and velocity of the dust. The canonical form of the dust action can be derived from the action for a continuously infinite number of relativistic point particles (Appendix D), and is a special case of the canonical action for arbitrary perfect fluids [16]. The Noether charges are represented by linear functionals of the dust momenta (Section 3b).

Dust is coupled to gravity by adding its action to the Dirac-ADM action. The dynamics is then generated by the standard super-Hamiltonian and supermomentum constraints on the phase space of the coupled system (Section 4a). The super-Hamiltonian and supermomentum constraints can be brought to a form in which they are resolved in the dust momenta. These new constraints $H_{\uparrow}(x)$ and $H_{\uparrow k}(x)$ generate an Abelian algebra (Section $4 \mathrm{~b})$ that allows us to represent diffeomorphisms in the dust spacetime by canonical trans- 
formations (Section 4c).

The new super-Hamiltonian constraint $H_{\uparrow}(x)$ generates dynamics along the flow lines of the dust, while the new supermomentum constraint $H_{\uparrow k}(x)$ generates motion along the hypersurfaces of constant dust proper time. The true Hamiltonian density in the superHamiltonian constraint is the square root of a quadratic combination $G(x)$ of the gravitational super-Hamiltonian and supermomentum. As such, it does not depend on the dust variables. The expressions $G(x)$ have vanishing Poisson brackets among themselves (Appendix E). When complemented by the gravitational supermomenta, they provide an alternative set of constraints for vacuum gravity which generate a true Lie algebra (Section $4 d)$.

The dust frame variables are fields $Z^{k}(x), k=1,2,3$, whose values at a given space point $x$ constitute a set of Lagrangian coordinate labels for the dust flow line that passes through $x$. $Z$ can be viewed as a mapping from the space manifold to the 'dust space' manifold whose points $z$ are the individual flow lines (Section $2 \mathrm{~b}$ ). Correspondingly, $Z$ induces mappings of tensor fields. In particular, the spatial metric, the dust proper time variable, and their canonical conjugates can be mapped from space to the dust space. This mapping of tensors is completed into a canonical transformation by replacing the momentum conjugate to the dust frame $Z^{k}(x)$ with the new supermomentum generator $H_{\uparrow k}(x)$ (Section 5a). Then the constraint $H_{\uparrow k}(x)=0$ is simply the statement that the momentum conjugate to $Z^{k}(x)$ should vanish. Because the new super-Hamiltonian constraint $H_{\uparrow}(x)$ is independent of the dust frame $Z$ and its conjugate, it can be expressed in terms of the new canonical variables by mapping the space scalar density $H_{\uparrow}(x)$ to the dust space. This turns the super-Hamiltonian constraint into a dust space scalar density $\boldsymbol{H}_{\uparrow}(z)$. The true Hamiltonian density in this constraint is the square root of a dust space density $\boldsymbol{G}(z)$ that is constructed solely from the gravitational variables. The Noether charges also can be expressed as functionals of the new canonical variables (Section $5 \mathrm{~b}$ ).

We quantize gravity coupled to dust by imposing the supermomentum constraint $H_{\uparrow k}(x)=0$ and the new super-Hamiltonian constraint $\boldsymbol{H}_{\uparrow}(z)=0$ as operator restrictions on the states (Section 6). The supermomentum operator constraint implies that the state functional is independent of $Z$, while the new Hamiltonian constraint yields a functional Schrödinger equation in the dust time (Section 6a). Since the Hamiltonian density in this equation does not depend on the dust variables, the dust time can be separated from the gravitational variables. This leads to a time-independent functional Schrödinger equation (Section $6 \mathrm{~b}$ ). If the operators $\hat{\boldsymbol{G}}(z)$ can be factor ordered and regularized so that they commute, then they possess joint eigenfunctionals (Section 6c). We show that the spectrum of $\hat{\boldsymbol{G}}(z)$ is continuous and degenerate (Appendix F). The square-root Hamiltonian density can be defined by spectral analysis on the Hilbert space $\mathcal{H}^{+}$spanned by the eigenfunctionals labeled by positive eigenvalues $\boldsymbol{G}(z)>0$. The Noether charges appear in the quantum theory as generators for unitary operators that transform the quantum states by the corresponding symmetries (Section 6d). The quantum theory of gravity and dust can be constructed using either the original set or the new set of canonical variables (Section 6g).

The Schrödinger equation yields a conserved inner product that can be written either in terms of the instantaneous state functionals, or as a product in the space of solutions (Section 6e). Gravitational observables admit a similar dual representation (Section 6f). Observables must keep the state in the Hilbert space $\mathcal{H}^{+}$. Examples of such observables are 
given; unfortunately, neither the intrinsic metric nor the extrinsic curvature are gravitational observables. A similar difficulty occurs already in a simple model system, namely, the relativistic particle in the proper-time formalism (Appendix G).

The classical Noether charges generate canonical transformations that transform the system from one dust time to another (Section $3 \mathrm{~b}$ ). They carry a solution of the constraints and of the Hamilton equations of motion again into a solution. If the dust does not rotate, the solution can be transformed to the time foliation that is orthogonal to the dust frame (Section 7a). The restriction to non-rotating dust in the frame-orthogonal time foliation can be enforced by an additional first-class constraint. This constraint can be used to eliminate the inconvenient square root in the Hamiltonian density $H_{\uparrow}(x)$ (Section $7 \mathrm{~b}$ ). The resulting Hamiltonian constraint $H_{\uparrow 0}(x)$ underlies our previous study of Gaussian reference fluids [10]. Due to factor-ordering ambiguities, the quantum theories constructed from $H_{\uparrow}(x)$ and $H_{\uparrow 0}(x)$ do not necessarily coincide (Section $7 \mathrm{c}$ ).

Our notation is summarized in Appendix A.

The introduction of dust alleviates several notorious problems of time in vacuum gravity: - When passing to the Schrödinger equation, one can rely on the privileged time and space brought into spacetime by the state of a material system.

- The dust time is a spacetime scalar. Its value does not depend on the hypersurface passing through a given event. No intrinsic time in vacuum gravity has this property.

- The Hamiltonian densities have vanishing Poisson brackets. If - and this is an overwhelming if - a factor ordering and regularization can be found for which the corresponding operators $\hat{\boldsymbol{G}}(z)$ commute, then the Hamiltonian density operators can be defined by spectral analysis and the evolution of states will be independent of the foliation connecting the final and initial embeddings. One important problem remains to be solved: What operators, if any, correspond to the measurements of the intrinsic metric and extrinsic curvature on an embedding specified with respect to the dust time and the dust frame?

To the extent limited by our cautionary remarks, the introduction of incoherent dust provides a satisfactory phenomenological approach to the problems of time in canonical quantum gravity. The corresponding problems at the level of vacuum gravity remain, of course, unresolved.

Preliminary accounts of our work have been given in Refs. [17].

\section{INCOHERENT DUST}

\section{A. Spacetime Action and the Euler Equations}

Dust is described by eight spacetime scalars, $T, Z^{k}, M$, and $W_{k}$. (Indices $k, \ell$ from the middle of the Latin alphabet take the values 1, 2,3.) The physical interpretation of these state variables follows from the analysis of the action and the ensuing equations of motion. In order to describe the structure of the action in physical terms, we shall anticipate the results of this analysis. The values of the variables $Z^{k}$ are the comoving coordinates of the dust particles, and the value of the variable $T$ is the proper time measured along the particle flow lines. The scalars $Z^{K}=\left(T, Z^{k}\right)$ are assumed to be four independent functions of the spacetime coordinates $y^{\alpha}, \alpha=0,1,2,3$ : 


$$
\operatorname{det}\left(Z_{, \alpha}^{K}\right) \neq 0
$$

The four-velocity $U_{\alpha}$ of the dust is defined by its decomposition in the cobasis $Z^{K}{ }_{, \alpha}$ as

$$
U_{\alpha}=-T_{, \alpha}+W_{k} Z_{, \alpha}^{k} .
$$

The three spatial components $W_{k}$ of the four-velocity in the dust frame $Z^{k}$ are also state variables. Equation (2.2) expresses the four-velocity one-form $\boldsymbol{U}=U_{\alpha} d y^{\alpha}$ as a Pfaff form 18

$$
\boldsymbol{U}=-d T+W_{k} d Z^{k}
$$

of the seven scalar fields $T, Z^{k}$ and $W_{k}$. The eighth state variable, $M$, is the rest mass density of the dust.

The dust action is a functional of the dust variables and of the spacetime metric $\gamma_{\alpha \beta}$,

$$
S^{D}\left[T, Z^{k} ; M, W_{k} ; \gamma_{\alpha \beta}\right]=\int_{\mathcal{M}} d^{4} y L^{D}(y)
$$

with the Lagrangian density

$$
L^{D}=-\frac{1}{2}|\gamma|^{1 / 2} M\left(\gamma^{\alpha \beta} U_{\alpha} U_{\beta}+1\right) .
$$

It can be cast into many alternative forms (Appendix B). The matter equations of motion are obtained by varying the dust action with respect to the state variables $M, W_{k}, T$, and $Z^{k}$ :

$$
\begin{aligned}
& 0=\frac{\delta S^{D}}{\delta M}=-\frac{1}{2}|\gamma|^{1 / 2}\left(\gamma^{\alpha \beta} U_{\alpha} U_{\beta}+1\right) \\
& 0=\frac{\delta S^{D}}{\delta W_{k}}=-|\gamma|^{1 / 2} M Z_{, \alpha}^{k} U^{\alpha} \\
& 0=\frac{\delta S^{D}}{\delta T}=-\left(|\gamma|^{1 / 2} M U^{\alpha}\right)_{, \alpha} \\
& 0=\frac{\delta S^{D}}{\delta Z^{k}}=\left(|\gamma|^{1 / 2} M W_{k} U^{\alpha}\right)_{, \alpha}
\end{aligned}
$$

These equations corroborate our interpretation of the dust variables:

\section{B. Dust As a Standard of Space and Time}

Equation (2.6) ensures that the four-velocity $U^{\alpha}$ is a unit timelike vector field. According to Eq. (2.7), the three scalar fields $Z^{k}$ are constant along the flow lines $U^{\alpha}$ and therefore their values $z^{k}$ can be interpreted as Lagrangian coordinates for the dust. That is, the values $z^{k}=Z^{k}(y)$ of the mappings $Z^{k}: \mathcal{M} \rightarrow \mathbb{R}$ serve as labels specifying which flow line passes through a given spacetime event $y$. Such a set of Lagrangian coordinates can be generated by choosing an arbitrary spacelike hypersurface $\Sigma$ and specifying a coordinate system $z^{k}$ on that hypersurface. Then each flow line is labeled by the coordinates of the point where it 
intersects the hypersurface. Inversely, knowing the congruence $\mathcal{S}$ of flow lines, we can define the mappings $Z^{k}$ of $\mathcal{M}$ into $\mathbb{R}$ by assigning to each event $y$ in $\mathcal{M}$ the coordinates $z^{k}$ of the point in $\Sigma$ where the flow line that passes through $y$ intersects $\Sigma$.

The congruence $\mathcal{S}$ of dust flow lines introduces a privileged reference frame into the spacetime manifold $\mathcal{M}$. This congruence can be viewed as an abstract three-dimensional space, the 'dust space', whose points are the individual flow lines. As a manifold, the dust space $\mathcal{S}$ is isomorphic to any spacelike hypersurface. (We assume that the spacetime $(\mathcal{M}$, $\gamma$ ) is globally hyperbolic with $\mathcal{M}=\mathbb{R} \times \Sigma$.) A system of coordinates on that hypersurface induces a system of coordinates $z^{k}$ on $\mathcal{S}$. It may be impossible to cover the dust space with a single coordinate chart, so the Lagrangian coordinates $z^{k}$ are generally defined only in open subsets of the dust space. Correspondingly, the scalar fields $Z^{k}$ are generally defined only in open tubes of the space-time manifold. Our interpretation of the fields $Z^{k}$ presupposes that their values constitute a good set of Lagrangian coordinates, i.e., that within the appropriate open tubes each flow line carries a unique set of labels $z^{k}$. As a consequence, the three gradients $Z^{k}{ }_{\alpha}$ must be linearly independent covectors. This is ensured by the assumption (2.1).

Our discussion reveals that the three scalar fields $Z^{k}(y)$ are not necessarily globally defined on the whole spacetime manifold $\mathcal{M}$. However, these fields appear in the action (2.4) only in the combination $W_{k} Z^{k}{ }_{\alpha}$, so this defect can be overcome by a slight reinterpretation of the formalism. Let $Z: \mathcal{M} \rightarrow \mathcal{S}$ denote a globally defined mapping of the spacetime $\mathcal{M}$ into the dust space $\mathcal{S}$, and view $Z^{k}$ as the composition of the mapping $Z$ with a local coordinate chart mapping on $\mathcal{S}$. With the indices $k$ and $\alpha$ interpreted as abstract tensor indices, $Z^{k}{ }_{, \alpha}$ is then globally defined as the mapping $Z^{k}{ }_{, \alpha}: \mathcal{M} \rightarrow T \mathcal{S} \otimes T^{*} \mathcal{M}$. Likewise, $W_{k}$ is defined as the mapping $W_{k}: \mathcal{M} \rightarrow T^{*} \mathcal{S}$ where, again, $k$ is viewed as an abstract index. In this way, $W_{k} Z^{k}, \alpha$ is understood to be a globally defined element of $T^{*} \mathcal{M}$.

The dust enables us to introduce not only the privileged matter space, but also a matter time. By multiplying Eq. (2.2) by $U^{\alpha}$ and using the field equations (2.6)-(2.7), we learn that

$$
£_{U} T=T_{, \alpha} U^{\alpha}=1,
$$

i.e., that $T$ is the proper time between a fiducial hypersurface $T=0$ and an arbitrary hypersurface $T=$ const along the flow lines of the dust particles. The fiducial hypersurface can be chosen to be spacelike. The foliation of the spacetime manifold $\mathcal{M}$ by hypersurfaces of constant $T$ can be viewed as an abstract one-dimensional space, the 'dust time' $\mathcal{T}$, whose points (instants) are the individual hypersurfaces. As a manifold, the dust time $\mathcal{T}$ is isomorphic to $\mathbb{R}$. The value $\tau=T(y)$ of the time mapping $T: \mathcal{M} \rightarrow \mathcal{T}$ can serve as the time coordinate of an instant of time that passes through the event $y$.

In our analysis, we must assume that the three covectors $Z^{k}{ }_{, \alpha}$ are linearly independent. That the fourth covector, $T_{, \alpha}$, is linearly independent of $Z^{k}{ }_{, \alpha}$ follows from the equations of motion (2.7) and (2.10). As a result, $\left(T_{, \alpha}, Z^{k}{ }_{, \alpha}\right)$ form a basis in $T^{*} \mathcal{M}$, Eq. (2.1). The four-velocity $U^{\alpha}$ of the dust is a privileged timelike future-pointing vector. Equation (2.10) tells us that the time function $T(y)$ grows from the past to the future. The covectors $Z^{k}{ }_{, \alpha}$ are orthogonal to the four-velocity $U^{\alpha}$ of the dust and hence spacelike. These relations are sketched in Fig. 1. 
Because $\left(T_{, \alpha}, Z^{k}{ }_{, \alpha}\right)$ are linearly independent, the values $\tau$ and $z^{k}$ of the four mappings $T$ and $Z^{k}$ can be used as coordinates on $\mathcal{M}$. By inverting the mappings

$$
\tau=T(y), \quad z=Z(y)
$$

we obtain the mapping

$$
\Upsilon: \mathcal{T} \times \mathcal{S} \rightarrow \mathcal{M} \quad \text { by } \quad(\tau, z) \mapsto y=\Upsilon(\tau, z) .
$$

The four vectors

$$
U^{\alpha}:=\Upsilon^{\alpha}{ }_{, \tau} \text { and } \Upsilon^{\alpha}{ }_{, k}
$$

form a basis in $T \mathcal{M}$ dual to the cobasis

$$
Z^{K}{ }_{, \alpha}=\left(T_{, \alpha}, Z^{k}{ }_{, \alpha}\right)
$$

in $T^{*} \mathcal{M}$. The basis (2.13) and the cobasis (2.14) satisfy the standard orthonormality and completeness relations. As a consequence of the equations of motion (2.7) and (2.10), these basis and cobasis vectors are conserved along the flow lines of $U^{\alpha}$; that is, $£_{U} T_{, \alpha}=0=$ $£_{U} Z^{k}{ }_{, \alpha}$ and $£_{U} U^{\alpha}=0=£_{U} Y^{\alpha}{ }_{, k}$. The basis vector $U^{\alpha}:=\Upsilon^{\alpha}{ }_{, \tau}$ coincides with the fourvelocity of the dust and the remaining three vectors, $\Upsilon^{\alpha}{ }_{, k}$, are tangent to the $T=$ const hypersurfaces. These relations are again sketched in Fig. 1.

To summarize, any solution of the dust equations (2.6) and (2.7) which satisfies the condition (2.1) describes a motion of the dust that allows the spacetime manifold $\mathcal{M}$ to be split into the space and time manifolds, $\mathcal{S}$ and $\mathcal{T}$. An instant of $\mathcal{T}$ and a flow line of $\mathcal{S}$ intersect at a unique event $y \in \mathcal{M}$. The spacetime $\mathcal{M}$ is thus a Cartesian product $\mathcal{T} \times \mathcal{S}$ of the space and time manifolds. The mapping $T(y) \times Z(y)$ takes us from $\mathcal{M}$ to $\mathcal{T} \times \mathcal{S}$, the inverse mapping $\Upsilon(\tau, z)$ takes us from $\mathcal{T} \times \mathcal{S}$ to $\mathcal{M}$. In this sense, dust serves as a standard of space and time.

\section{Conservation Laws}

Let us now turn to the second set of Euler equations. Equation (2.8) is a continuity equation for the rest mass current

$$
J^{\alpha}:=M U^{\alpha} .
$$

The total mass is thus conserved from one spacelike hypersurface to another. Similarly, the Euler equation (2.9) is a continuity equation for

$$
J_{k}^{\alpha}:=W_{k} J^{\alpha},
$$

which we refer to as the momentum current. These two continuity equations imply

$$
£_{U} W_{k}=W_{k, \alpha} U^{\alpha}=0,
$$

so that $W_{k}$ stays the same along each flow line. As a result, the velocity $W_{k}$ can depend on $y$ only through the Lagrangian coordinates $z=Z(y)$ :

$$
W_{k}(y)=\boldsymbol{W}_{k}(Z(y)) .
$$

This shows that $W_{k}$ can be viewed as a covector, or one-form, in the dust space $\mathcal{S}$. 


\section{The Energy-Momentum Tensor}

Dust is coupled to the metric field by adding its action $S^{D}$ to the Hilbert action $S^{G}$, which is given by

$$
S^{G}\left[\gamma_{\alpha \beta}\right]=\int_{\mathcal{M}} d^{4} y L^{G} \quad, \quad L^{G}=|\gamma|^{1 / 2} R\left(y ; \gamma_{\alpha \beta}\right]
$$

The variation of the total action $S$ with respect to $\gamma_{\alpha \beta}$ yields the Einstein law of gravitation

$$
R^{\alpha \beta}-\frac{1}{2} R \gamma^{\alpha \beta}=\frac{1}{2} T^{\alpha \beta}
$$

with the energy-momentum tensor

$$
\begin{aligned}
T^{\alpha \beta}:=2|\gamma|^{1 / 2} \frac{\delta S^{D}}{\delta \gamma_{\alpha \beta}} & =M U^{\alpha} U^{\beta}+\frac{1}{2} \gamma^{\alpha \beta} M\left(\gamma^{\mu \nu} U_{\mu} U_{\nu}+1\right) \\
& \approx M U^{\alpha} U^{\beta} .
\end{aligned}
$$

Modulo the normalization equation (2.6), this tensor has the form required for the description of incoherent dust with rest mass density $M$ and four-velocity $U^{\alpha}$.

By the well-known argument, the invariance of the action (2.4)-(2.5) under spacetime diffeomorphisms $\varphi$, namely,

$$
S^{D}\left[\varphi^{*} T, \varphi^{*} Z^{k} ; \varphi^{*} M, \varphi^{*} W_{k} ; \varphi^{*} \gamma_{\alpha \beta}\right]=S^{D}\left[T, Z^{k} ; M, W_{k} ; \gamma_{\alpha \beta}\right]
$$

leads to the identity

$$
|\gamma|^{1 / 2} \nabla_{\beta} T_{\alpha}^{\beta} \equiv \frac{\delta S^{D}}{\delta T} T_{, \alpha}+\frac{\delta S^{D}}{\delta Z^{k}} Z_{, \alpha}^{k}+\frac{\delta S^{D}}{\delta M} M_{, \alpha}+\frac{\delta S^{D}}{\delta W_{k}} W_{k, \alpha}
$$

It follows that if the dust satisfies the field equations (2.6)-(2.9), the energy-momentum tensor is covariantly conserved:

$$
\nabla_{\beta} T_{\alpha}^{\beta}=0
$$

The conservation law (2.24) also follows independently of the Euler equations (2.6)-(2.9) from the Einstein law of gravitation (2.20) through the contracted Bianchi identities. The identity (2.23) shows that if we postulate the conservation law (2.24), the Euler equations (2.6)-(2.9) are not independent. It suffices to impose the first set of equations, (2.6) and (2.7), obtained by varying the multipliers $M$ and $W_{k}$. The second set of Euler equations, (2.8) and (2.9), obtained by varying the dynamical variables $T$ and $Z^{k}$, then follows because $\left(T_{, \alpha}, Z^{k}, \alpha\right)$ is a regular cobasis.

The first and second sets of Euler equations have a complementary character. The first set, obtained by varying the multipliers $M$ and $W_{k}$, tells us that the dynamical variables $T$ and $Z^{k}$ have the properties (2.10) and (2.7) of the proper time and the Lagrangian coordinates of the dust. The second set, obtained by varying the dynamical variables $T$ and $Z^{k}$, tells us that the multipliers $M$ and $W_{k}$ satisfy conservation laws (2.8) and (2.9) (or 
(2.17)). In the canonical formalism, the state variables $T$ and $Z^{k}$ are turned into canonical coordinates, while the Lagrange multipliers $M$ and $W_{k}$ are combined to form the conjugate momenta.

From the energy-momentum conservation (2.24) and the mass conservation (2.8) it follows that the flow lines of the dust particles are geodesics,

$$
U^{\beta} \nabla_{\beta} U^{\alpha}=0
$$

This demonstrates that the dust particles do not act on one another by mechanical forces.

\section{E. Symmetries and Noether Charges}

The action (2.4)-(2.5) depends on the variables $T, Z^{k}$, and $W_{k}$ only through the combination that occurs in the Pfaff form (2.3). It follows that invertible transformations of these variables that leave the Pfaff form unchanged constitute symmetries of the dust action. In Appendix $\mathrm{C}$ we identify two particular types of symmetry transformations that mix the dust spacetime variables among themselves. The first is expressed as

$$
Z^{k}=\Xi^{k}\left(Z^{m^{\prime}}\right), \quad W_{k^{\prime}}=\Xi_{, k^{\prime}}^{\ell}\left(Z^{m^{\prime}}\right) W_{\ell}
$$

These transformations are diffeomorphisms of the dust space $\mathcal{S}$, where the velocity $W_{k}$ transforms as a covector. Under the transformations (2.26), the dust worldline that passes through the spacetime point $y$ has its Lagrangian coordinate labels changed from $z^{k}=Z^{k}(y)$ to $z^{k^{\prime}}=Z^{k^{\prime}}(y)$.

The second type of symmetry transformation is expressed as

$$
T^{\prime}=T+\Theta\left(Z^{\ell}\right), \quad W_{k^{\prime}}=W_{k}+\Theta_{, k}\left(Z^{\ell}\right)
$$

The dust time $\mathcal{T}$ is not entirely determined by the dust flow lines since the initial hypersurface can be chosen arbitrarily. Once an initial hypersurface is chosen, all other hypersurfaces of the time foliation are determined, according to Eq. (2.10), by Lie propagation of the initial hypersurface along the flow lines of $U^{\alpha}$. If the initial hypersurface is changed, the whole foliation $\mathcal{T}$ is changed. Equation (2.27) connects the time function $T(y)$ of the old foliation $T(y)=\tau=$ const with the time function $T^{\prime}(y)$ of the new foliation $T^{\prime}(y)=\tau^{\prime}=$ const. It also determines how the projection $W_{k}$ of $U^{\alpha}$ onto the leaves of the time foliation is affected by a different tilt of the leaves with respect to the flow lines.

The transformations (2.26)-(2.27) are global symmetries of the action, because they do not depend explicitly on $y$ and therefore cannot be made to vanish in a compact region of $\mathcal{M}$. (Gauge symmetries, on the other hand, vary with $y$.) These symmetry transformations carry one solution of the field equations into another solution. Indeed, it is easy to check that if the field equations (2.7) and (2.9) - the only ones that contain the relevant state variables - are satisfied for the original variables, they are also satisfied for the transformed variables.

According to Noether's theorem, to each one-parameter group of symmetry transformations connected to the identity there corresponds a conserved charge. Let $\psi_{r}(y)$ denote a 
collection of field variables and $\psi_{r}^{\prime}=\Psi_{r}(\psi, \sigma)$ denote a one-parameter group of symmetries with the identity at $\sigma=0$. The corresponding conserved charge $Q$ is given by the integral

$$
Q=\int_{\Sigma} d \sum_{\alpha} \widetilde{J}^{\alpha}
$$

of the Noether current

$$
\widetilde{J}^{\alpha}:=\left.\frac{\partial L}{\partial \psi_{r, \alpha}} \frac{\partial \Psi_{r}(\psi, \sigma)}{\partial \sigma}\right|_{\sigma=0} .
$$

The integral in Eq. (2.28) is over an arbitrary spacelike hypersurface $Y(\Sigma)$ specified by an embedding

$$
Y: \Sigma \rightarrow \mathcal{M} \quad \text { by } \quad x \in \Sigma \mapsto y=Y(x)
$$

and carrying the oriented surface element

$$
d{\underset{\sim}{\alpha}}_{\alpha}:=\underset{\sim}{\delta} \alpha \beta \gamma \delta Y_{, b}^{\beta} Y_{, c}^{\gamma} Y_{, d}^{\delta} d x^{b} \wedge d x^{c} \wedge d x^{d}
$$

(Note that $\widetilde{J}^{\alpha}$ is a spacetime vector density of weight 1 , and $d \sum_{\alpha}$ is a covector density of the opposite weight -1 due to the presence of the alternating symbol $\underset{\sim}{\sim} \alpha \beta \gamma \delta$.)

The symmetry transformations (2.26)-(2.27) contain arbitrary functions $\Xi^{k}\left(Z^{\ell^{\prime}}\right)$ and $\Theta\left(Z^{\ell}\right)$. The Lie algebra of their one-parameter subgroups is thus labeled by the arbitrary functions

$$
\boldsymbol{\xi}^{k}\left(Z^{\ell}\right):=\left.\frac{\partial \boldsymbol{\Xi}^{k}\left(Z^{\ell^{\prime}}, \sigma\right)}{\partial \sigma}\right|_{\sigma=0}, \quad \boldsymbol{\vartheta}\left(Z^{\ell}\right):=\left.\frac{\partial \boldsymbol{\Theta}\left(Z^{\ell}, \sigma\right)}{\partial \sigma}\right|_{\sigma=0}
$$

For the symmetry transformations (2.26), the corresponding Noether currents (2.29) are

$$
\widetilde{J}^{\alpha}[\overrightarrow{\boldsymbol{\xi}}]=|\gamma|^{1 / 2} J_{k}^{\alpha} \boldsymbol{\xi}^{k}(Z)
$$

where $J_{k}^{\alpha}$ is the momentum current (2.16). For the symmetry transformations $(2.27)$, the corresponding Noether currents are

$$
\widetilde{J}^{\alpha}[\boldsymbol{\vartheta}]=|\gamma|^{1 / 2} J^{\alpha} \boldsymbol{\vartheta}(Z)
$$

where $J^{\alpha}$ is the mass current (2.15). By virtue of the field equations (2.7)-(2.9), the Noether currents satisfy the continuity equations

$$
\widetilde{J}_{, \alpha}^{\alpha}[\overrightarrow{\boldsymbol{\xi}}]=0=\widetilde{J}_{, \alpha}^{\alpha}[\boldsymbol{\vartheta}]
$$

The Noether charges (2.28) can be written in the form

$$
\begin{aligned}
Q[\overrightarrow{\boldsymbol{\xi}}] & =-\int_{\Sigma} d^{3} x|g|^{1 / 2} n_{\alpha} J_{k}^{\alpha} \boldsymbol{\xi}^{k}(Z(x)), \\
Q[\boldsymbol{\vartheta}] & =-\int_{\Sigma} d^{3} x|g|^{1 / 2} n_{\alpha} J^{\alpha} \boldsymbol{\vartheta}(Z(x)),
\end{aligned}
$$


where $|g|^{1 / 2} d^{3} x$ is the proper volume element of $Y(\Sigma)$ and $n_{\alpha}$ is the future-pointing unit normal.

In order to interpret the charge (2.37), choose $\boldsymbol{\vartheta}$ to be unity for all $Z^{k}$ in some ball $\mathcal{B}$ in the dust space $\mathcal{S}$, and zero outside $\mathcal{B}$. Then $Q[\boldsymbol{\vartheta}]$ becomes a proper volume integral over the subspace of the hypersurface $Y(\Sigma)$ that contains flow lines in $\mathcal{B}$, with the integrand $M\left(-n_{\alpha} U^{\alpha}\right)$. The factor $-n_{\alpha} U^{\alpha}$ is just the relativistic 'gamma factor' characterizing a boost from the Lagrangian observers whose four-velocities are $U^{\alpha}$ to the Eulerian observers on $Y(\Sigma)$ whose four-velocities are $n^{\alpha}$. Thus, $Q[\boldsymbol{\vartheta}]$ is the rest mass in $\mathcal{B}$ as seen by the Eulerian observers. Conservation of the charge (2.37) expresses the conservation of relativistic mass within a flow tube defined by the bundle of flow lines crossing the ball $\mathcal{B}$. For $\mathcal{B}=\Sigma$, conservation of the total relativistic mass is obtained.

Similarly, choose $\boldsymbol{\xi}^{k}=\delta_{\ell}^{k}$ in the ball $\mathcal{B}$, and zero outside $\mathcal{B}$. The charge (2.36) has the meaning of minus the component of dust momentum along the coordinate line $z^{\ell}$ in the $T=$ const surface within $\mathcal{B}$.

\section{CANONICAL DESCRIPTION OF DUST}

\section{A. The Canonical Action}

The ADM decomposition of the dust action follows the familiar pattern. Foliate spacetime into spacelike hypersurfaces by

$$
Y: \mathbb{R} \times \Sigma \rightarrow \mathcal{M} \quad \text { by } \quad(t, x) \mapsto y=Y(t, x),
$$

a one-parameter family of embeddings of space $\Sigma$ into spacetime $\mathcal{M}$. In local coordinates $x^{a}$ on $\Sigma$ and $y^{\alpha}$ on $\mathcal{M}$, the foliation (3.1) is represented by

$$
\left(t, x^{a}\right) \mapsto y^{\alpha}=Y^{\alpha}\left(t, x^{a}\right)
$$

A transition from one leaf of the foliation to another is described by the deformation vector $N^{\alpha}:=\dot{Y}^{\alpha}$. Its decomposition

$$
N^{\alpha}:=\dot{Y}^{\alpha}=N^{\perp} n^{\alpha}+N^{a} Y_{, a}^{\alpha}
$$

into the unit normal $n^{\alpha}$ and tangential $Y_{, a}^{\alpha}$ directions yields the lapse function $N^{\perp}$ and the shift vector $N^{a}$. The spacetime metric $\gamma_{\alpha \beta}$ is determined by

$$
\gamma^{\alpha \beta}=-n^{\alpha} n^{\beta}+g^{a b} Y_{, a}^{\alpha} Y_{, b}^{\beta}, \quad|\gamma|^{1 / 2}=N^{\perp}|g|^{1 / 2}
$$

where $g^{a b}$ is the inverse of the spatial metric $g_{a b}$ induced on $\Sigma$.

Scalar fields on $\mathcal{M}$, such as $Z^{k}$, can be pulled back to $\mathbb{R} \times \Sigma$ by the mapping (3.1). Then, according to Eq. (3.3),

$$
Z_{, \alpha}^{k} n^{\alpha}=\left(N^{\perp}\right)^{-1}\left(\dot{Z}^{k}-Z_{, a}^{k} N^{a}\right) .
$$

Similar equations hold for the scalar fields $T, M$, and $W_{k}$. From a global point of view, the mapping (3.1) is used to pull back the mapping $Z: \mathcal{M} \rightarrow \mathcal{S}$ to $\mathbb{R} \times \Sigma$, which yields a 
one-parameter family of mappings $Z_{t}: \Sigma \rightarrow \mathcal{S}$ from space $\Sigma$ into the dust space $\mathcal{S}$. (We will often suppress the $t$-dependence and simply write $Z: \Sigma \rightarrow \mathcal{S}$.) With the indices $k$ and $a$ viewed as abstract indices, $\dot{Z}^{k}$ denotes a one-parameter family of mappings from $\Sigma$ to $T \mathcal{S}$. Likewise, $Z^{k}{ }_{, a}$ denotes a one-parameter family of mappings from $\Sigma$ to $T \mathcal{S} \otimes T^{*} \Sigma$ and $W_{k}$ denotes a one-parameter family of mappings from $\Sigma$ to $T^{*} \mathcal{S}$.

The results above enable the dust action (2.4)-(2.5) to be written as an integral over $\mathbb{R} \times \Sigma$,

$$
S^{D}\left[T, Z^{k} ; M, W_{k} ; g_{a b}, N^{\perp}, N^{a}\right]=\int_{\mathbb{R}} d t \int_{\Sigma} d^{3} x L^{D}
$$

where

$$
L^{D}=\frac{1}{2}|g|^{1 / 2} M\left(\frac{1}{N^{\perp}}\left(-\dot{T}+W_{k} \dot{Z}^{k}-U_{a} N^{a}\right)^{2}-N^{\perp}\left(g^{a b} U_{a} U_{b}+1\right)\right),
$$

is the dust Lagrangian density and $U_{a}:=U_{\alpha} Y^{\alpha}{ }_{, a}=-T_{, a}+W_{k} Z^{k}{ }_{, a}$.

Let us introduce the momentum $P$ conjugate to $T$ by

$$
P:=\frac{\partial L^{D}}{\partial \dot{T}}=|g|^{1 / 2} M\left(N^{\perp}\right)^{-1}\left(\dot{T}-W_{k} \dot{Z}^{k}+U_{a} N^{a}\right) .
$$

The physical meaning of $P$ is revealed when we identify the relativistic 'gamma factor' $-U_{\alpha} n^{\alpha}$ characterizing a boost from the Lagrangian observers whose four-velocities are $U^{\alpha}$, to the Eulerian observers on $Y(\Sigma)$ whose four-velocities are $n^{\alpha}$. From Eq. (2.2) for $U_{\alpha}$ and Eqs. (3.5) for $Z_{, \alpha}^{k} n^{\alpha}$ and $T_{, \alpha} n^{\alpha}$ we obtain

$$
-U_{\alpha} n^{\alpha}=\left(N^{\perp}\right)^{-1}\left(\dot{T}-W_{k} \dot{Z}^{k}+U_{a} N^{a}\right) .
$$

This shows that the canonical momentum $P$ is equal to the projection

$$
P=|g|^{1 / 2}\left(-J^{\alpha} n_{\alpha}\right)
$$

of the rest mass four-current (2.15) into the four-velocity of the Eulerian observers. Therefore $P$ is the density of rest mass on $Y(\Sigma)$, per unit coordinate cell $d^{3} x$ (rather than per unit proper volume $d V=|g|^{1 / 2} d^{3} x$ ), as measured by the Eulerian observers. Equation (3.10) also shows that the momentum $P$ is necessarily positive,

$$
P>0
$$

since $J^{\alpha}$ and $n^{\alpha}$ are both future-pointing timelike vectors.

Perform now the Legendre dual transformation $(T, \dot{T}) \rightarrow(T, P)$ while leaving the variables $Z^{k}, M$, and $W_{k}$ untouched. At the same time, introduce the abbreviation

$$
P_{k}:=-P W_{k}
$$

for the Lagrange multiplier $W_{k}$ scaled by $-P$. The resulting Hamiltonian $P \dot{T}-L^{D}$ is

$$
P \dot{T}-L^{D}=-P_{k} \dot{Z}^{k}+N^{\perp} H_{\perp}^{D}+N^{a} H_{a}^{D}
$$


with

$$
H_{a}^{D}:=-P U_{a}=P T_{, a}+P_{k} Z_{, a}^{k}
$$

and

$$
H_{\perp}^{D}:=\frac{1}{2} \frac{P^{2}}{M|g|^{1 / 2}}+\frac{1}{2} \frac{M|g|^{1 / 2}}{P^{2}}\left(P^{2}+g^{a b} H_{a}^{D} H_{b}^{D}\right) .
$$

Equation (3.13) reveals that the Legendre transformed action

$$
\begin{aligned}
S^{D}\left[T, P ; Z^{k},\right. & \left.P_{k} ; M ; g_{a b}, N^{\perp}, N^{a}\right] \\
& =\int_{\mathbb{R}} d t \int_{\Sigma} d^{3} x\left(P \dot{T}+P_{k} \dot{Z}^{k}-N^{\perp} H_{\perp}^{D}-N^{a} H_{a}^{D}\right)
\end{aligned}
$$

is in canonical form not only in the variables $T$ and $P$, but also in the variables $Z^{k}$ and $P_{k}$. The variable (3.12) is thus the momentum canonically conjugate to $Z^{k}$. As a pendant to Eq. (3.10), the momentum $P_{k}$ of Eq. (3.12) can be expressed as

$$
P_{k}=-|g|^{1 / 2}\left(-J_{k}^{\alpha} n_{\alpha}\right)
$$

and interpreted as (minus) the momentum density along $\Upsilon^{\alpha}{ }_{, k}$ per unit coordinate cell $d^{3} x$, measured by the Eulerian observers on $Y(\Sigma)$.

By varying the action (3.16) with respect to $M$ we get

$$
\frac{\delta S^{D}}{\delta M}=-N^{\perp} \frac{\partial H_{\perp}^{D}}{\partial M}=0 \Longrightarrow \quad M=\frac{1}{|g|^{1 / 2}} \frac{P^{2}}{\sqrt{P^{2}+g^{a b} H_{a}^{D} H_{b}^{D}}} .
$$

The positive square root is dictated by the positivity of $M$. Substituting the expression (3.18) back into $H_{\perp}^{D}$ gives

$$
H_{\perp}^{D}=\sqrt{P^{2}+g^{a b} H_{a}^{D} H_{b}^{D}}
$$

and eliminates the mass multiplier $M$ from the action. Equations (3.12) and (3.18) connect the multiplier variables $M, W_{k}$ of the spacetime action with the canonical momenta $P, P_{k}$.

The spatial densities $H_{a}^{D}$ and $H_{\perp}^{D}$ are, respectively, minus the momentum density and energy density (in the coordinate basis $d x^{a}$ ) of dust as seen by the Eulerian observers $n^{\alpha}$ on $Y(\Sigma)$. The canonical form (3.14) of $H_{a}^{D}$ is dictated by its role as the canonical generator of spatial diffeomorphisms for the spatial scalars $T, Z^{k}$ and their conjugates $P, P_{k}$. Very few field theoretical systems have a square-root energy density like $H_{\perp}^{D}$. (Two such systems known to us are the Born-Infeld electrodynamics [19] and the extrinsic time reference fluid |13.) The square-root structure of the dust Hamiltonian (3.19) arises from the square-root structure of the Hamiltonian of a single relativistic particle. This connection is demonstrated in Appendix D, which makes use of results from Sec. 5.

Because the dust action contains no derivatives of the metric, the ADM decomposition of the total action leads to the super-Hamiltonian and supermomentum constraints 


$$
\begin{aligned}
H_{\perp} & :=H_{\perp}^{G}+H_{\perp}^{D}=0, \\
H_{a} & :=H_{a}^{G}+H_{a}^{D}=0,
\end{aligned}
$$

whose gravitational contributions $H_{\perp}^{G}$ and $H_{a}^{G}$ have the same form as in vacuum general relativity, namely,

$$
\begin{aligned}
H_{\perp}^{G}\left(x ; g_{a b}, p^{a b}\right] & =G_{a b c d}(x ; g) p^{a b}(x) p^{c d}(x)-|g|^{1 / 2} R(x ; g], \\
G_{a b c d} & =\frac{1}{2}|g|^{-1 / 2}\left(g_{a c} g_{b d}+g_{a d} g_{b c}-g_{a b} g_{c d}\right), \\
H_{a}^{G}\left(x ; g_{a b}, p^{a b}\right] & =-2 D_{b} p_{a}^{b}(x) .
\end{aligned}
$$

The total constraints (3.20) and (3.21) satisfy the usual Dirac 'algebra' (Sec. 4a).

\section{B. Symmetries and Charges}

By using Eqs. (3.10) and (3.17), the Noether charges (2.36) and (2.37) can be expressed as functionals of the canonical variables,

$$
\begin{aligned}
& Q[\overrightarrow{\boldsymbol{\xi}}]=-\int_{\Sigma} d^{3} x \boldsymbol{\xi}^{k}(Z(x)) P_{k}(x), \\
& Q[\boldsymbol{\vartheta}]=\int_{\Sigma} d^{3} x \boldsymbol{\vartheta}(Z(x)) P(x) .
\end{aligned}
$$

The global symmetries of Section 2e appear in the canonical formalism as canonical transformations of the dynamical variables generated through the Poisson brackets by the Noether charges (3.24) and (3.25). For example, by applying the canonical transformation generated by the charge (3.25) to the dynamical variables $T(x)$ and

$$
W_{k}(x):=-P_{k}(x) / P(x),
$$

the infinitesimal version of Eq. (2.27) is recovered:

$$
\{T(x), Q[\boldsymbol{\vartheta}]\}=\boldsymbol{\vartheta}(Z(x)), \quad\left\{W_{k}(x), Q[\boldsymbol{\vartheta}]\right\}=\boldsymbol{\vartheta}_{, k}(Z(x)) .
$$

Likewise, the canonical transformation generated by the charge (3.24) yields the infinitesimal version of Eq. (2.26).

The phase space functionals (3.24), (3.25) have vanishing Poisson brackets with the constraints (3.20) and (3.21), so they indeed generate symmetries of the theory. By the same token, they are constants of motion. The symmetry generators (3.24) and (3.25) close under the Poisson brackets according to the equations

$$
\begin{aligned}
\left\{Q\left[\overrightarrow{\boldsymbol{\xi}}_{1}\right], Q\left[\overrightarrow{\boldsymbol{\xi}}_{2}\right]\right\} & =Q\left[£_{\overrightarrow{\boldsymbol{\xi}}_{1}} \overrightarrow{\boldsymbol{\xi}}_{2}\right], \\
\{Q[\overrightarrow{\boldsymbol{\xi}}], Q[\boldsymbol{\vartheta}]\} & =Q\left[£_{\overrightarrow{\boldsymbol{\xi}}} \boldsymbol{\vartheta}\right], \\
\left\{Q\left[\boldsymbol{\vartheta}_{1}\right], Q\left[\boldsymbol{\vartheta}_{2}\right]\right\} & =0 .
\end{aligned}
$$

Thus, the $Q$ 's form a realization of the Lie algebra of the subgroup (2.26)-(2.27) of diffeomorphisms of the dust spacetime $\mathcal{T} \times \mathcal{S}$. 


\section{THE CONSTRAINT ALGEBRA}

\section{A. The Dirac Algebra of Old Constraints}

The constraints (3.20)-(3.21) of dust coupled to gravity satisfy the usual Dirac 'algebra'

$$
\begin{aligned}
& \left\{H_{\perp}(x), H_{\perp}\left(x^{\prime}\right)\right\}=g^{a b}(x) H_{b}(x) \delta_{, a}\left(x, x^{\prime}\right)-\left(x \leftrightarrow x^{\prime}\right), \\
& \left\{H_{\perp}(x), H_{a}\left(x^{\prime}\right)\right\}=-H_{\perp}\left(x^{\prime}\right) \delta_{, a^{\prime}}\left(x, x^{\prime}\right) \\
& \left\{H_{a}(x), H_{b}\left(x^{\prime}\right)\right\}=H_{b}(x) \delta_{, a}\left(x, x^{\prime}\right)-\left(a x \leftrightarrow b x^{\prime}\right) .
\end{aligned}
$$

Equation (4.3) tells us that $H_{a}(x)$ represent the generators of the spatial diffeomorphism group Diff $\Sigma$. Equation (4.2) expresses the fact that the super-Hamiltonian $H_{\perp}(x)$ is a scalar density of weight 1 under Diff $\Sigma$. The right-hand-side of Eq. (4.1) contains the dynamical variable $g_{a b}(x)$. This implies that the total system $(4.1)-(4.3)$ is not a true Lie algebra [20].

The gravitational super-Hamiltonian $H_{\perp}^{G}(x)$ and supermomentum $H_{a}^{G}(x)$ close according to the same relations (4.1)-(4.3) as do the complete constraints $H_{\perp}(x)$ and $H_{a}(x)$. From here, one can deduce the Poisson brackets among the matter parts $H_{\perp}^{D}(x)$ and $H_{a}^{D}(x)$ of the constraints [21]. The momentum densities $H_{a}^{D}(x)$ still represent Diff $\Sigma$, Eq. (4.3). For systems with non-derivative gravitational coupling (which is the case for dust), the energy density $H_{\perp}^{D}(x)$ depends only on the metric $g_{a b}(x)$, but not on its derivatives. It follows that $H_{\perp}^{D}(x)$ and $H_{\perp}^{D}\left(x^{\prime}\right)$ close in the same manner, Eq. (4.1), as the total constraints $H_{\perp}(x)$ and $H_{\perp}\left(x^{\prime}\right)$, or as the gravitational expressions $H_{\perp}^{G}(x)$ and $H_{\perp}^{G}\left(x^{\prime}\right)$. The Poisson bracket $\left\{H_{\perp}^{D}(x), H_{a}^{D}\left(x^{\prime}\right)\right\}$ does not follow the pattern (4.2) because $H_{a}^{D}\left(x^{\prime}\right)$ does not act on the metric in $H_{\perp}^{D}(x)$. The concrete form of this bracket will not concern us here.

The energy density $H_{\perp}^{D}(x)$ of the dust is the square root of the expression

$$
D(x):=P^{2}(x)+g^{a b}(x) H_{a}^{D}(x) H_{b}^{D}(x) .
$$

From the closing relations $(4.1)$ for $H^{D}(x)$ it follows that

$$
\left\{D(x), D\left(x^{\prime}\right)\right\}=4 D(x) H^{D a}(x) \delta_{, a}\left(x, x^{\prime}\right)-\left(x \leftrightarrow x^{\prime}\right) .
$$

\section{B. The Abelian Algebra of New Constraints}

The fact that the Dirac 'algebra' is not a true Lie algebra creates difficulties in canonical quantum gravity. Isham and Kuchař [20] found a way in which the Dirac 'algebra' of gravity modified by the imposition of Gaussian coordinate conditions can be turned into a true Lie algebra. Kuchař and Torre [10] showed that this procedure is physically equivalent to the coupling of gravity to a Gaussian reference fluid that has the structure of a non-rotating, heat-conducting dust. We are presently dealing with a simpler and more natural matter source, namely, a general incoherent dust; however, the algorithm to be followed is essentially the same. The first step is to replace the old constraints (3.20)-(3.21) by an equivalent set of constraints based on the resolution of the old constraints with respect to the momenta $P$ and $P_{k}$ conjugate to the time $T$ and frame $Z^{k}$ variables. This task is simplified by the fact that 
the energy density $H_{\perp}^{D}(x)$ depends on $P_{k}(x)$ only through the momentum density $H_{a}^{D}(x)$. Hence, by using the supermomentum constraint (3.21), the super-Hamiltonian constraint (3.20) can be cast into the form

$$
H_{\uparrow}(x):=P(x)+h\left(x ; g_{a b}, p^{a b}\right]=0,
$$

with

$$
\begin{aligned}
h(x) & =-\sqrt{G(x)} \\
G\left(x ; g_{a b}, p^{a b}\right] & :=\left(H_{\perp}^{G}(x)\right)^{2}-g^{a b}(x) H_{a}^{G}(x) H_{b}^{G}(x) .
\end{aligned}
$$

The choice of the negative square root in Eq. (4.7a) is mandated by the positivity of $P(x)$, Eq. (3.11). Inversely, the positivity of $P(x)$ is a consequence of the new constraint (4.6). By introducing the inverse $Z_{k}^{a}(x)$ to the matrix $Z^{k}{ }_{, a}(x)$,

$$
Z_{, a}^{\ell}(x) Z_{k}^{a}(x)=\delta_{k}^{\ell},
$$

the supermomentum constraint (3.21) can be rewritten in the form

$$
\begin{aligned}
H_{\uparrow k}(x) & :=H_{a}(x) Z_{k}^{a}(x)=P_{k}(x)+h_{k}\left(x ; T, Z^{k}, g_{a b}, p^{a b}\right]=0, \\
h_{k}\left(x ; T, Z^{k}, g_{a b}, p^{a b}\right] & =Z_{k}^{a}(x) H_{a}^{G}(x)+\sqrt{G(x)} T_{, a}(x) Z_{k}^{a}(x),
\end{aligned}
$$

in which $P(x)$ has been eliminated by the use of Eq. (4.6).

The old constraints (3.20)-(3.21) and the new constraints (4.6), (4.9) are completely equivalent (in the region $G(x)>0, H_{\perp}^{G}(x)<0$ of the gravitational phase space). However, the momenta $P_{K}=\left(P, P_{k}\right)$ in Eqs. (4.6) and (4.9) are separated from the rest of the canonical variables. This has an important consequence for the constraint algebra, namely, that the Poisson brackets of the new constraint functions $H_{\uparrow K}(x)=\left(H_{\uparrow}(x), H_{\uparrow k}(x)\right)$ must strongly vanish:

$$
\left\{H_{\uparrow J}(x), H_{\uparrow K}\left(x^{\prime}\right)\right\}=0 .
$$

This remarkable fact follows by a simple argument [10] that circumvents the tedious algebra: Because the new constraints are equivalent to the old constraints and the Poisson brackets of the old constraints weakly vanish, the Poisson brackets of the new constraints also must weakly vanish. However, because the momenta $P_{K}$ appear in the constraints without any coefficients, these brackets cannot depend on $P_{K}$. Thus, the new constraints cannot help in any way to turn those brackets into zeros; this implies that the brackets must vanish strongly.

Thus, the new constraints generate an Abelian algebra (4.11). Let us smear these constraints by a set $N^{\uparrow K}(x)=\left(N^{\uparrow}(x), N^{\uparrow k}(x)\right)$ of externally prescribed scalar functions:

$$
\begin{aligned}
& H\left[N^{\uparrow}\right]:=\int_{\Sigma} d^{3} x N^{\uparrow}(x) H_{\uparrow}(x), \\
& H\left[\vec{N}^{\uparrow}\right]:=\int_{\Sigma} d^{3} x N^{\uparrow k}(x) H_{\uparrow k}(x) .
\end{aligned}
$$


The smeared constraints generate through the Poisson brackets the dynamical changes of the field variables $T, Z^{k}, P, P_{k}, g_{a b}$, and $p^{a b}$. In particular, $H\left[N^{\uparrow}\right]$ generates the change

$$
\begin{aligned}
\dot{T}(x) & :=\left\{T(x), H\left[N^{\uparrow}\right]\right\}=N^{\uparrow}(x), \\
\dot{Z}^{k}(x) & :=\left\{Z^{k}(x), H\left[N^{\uparrow}\right]\right\}=0,
\end{aligned}
$$

and $H\left[\vec{N}^{\uparrow}\right]$ generates the change

$$
\begin{aligned}
\dot{T}(x) & :=\left\{T(x), H\left[\vec{N}^{\uparrow}\right]\right\}=0, \\
\dot{Z}^{k}(x) & :=\left\{Z^{k}(x), H\left[\vec{N}^{\uparrow}\right]\right\}=N^{\uparrow k}(x),
\end{aligned}
$$

of the dust coordinates $T(x)$ and $Z^{k}(x)$. Equations (4.14) tell us that $H\left[N^{\uparrow}\right]$ displaces the hypersurface by the proper time $N^{\uparrow}(x)$ along the flow lines of the dust (Fig. 2). The Poisson bracket of any remaining variable (like $\left.g_{a b}(x)\right)$ with $H\left[N^{\uparrow}\right]$ then gives the change of that variable under such a displacement. Equation (4.15) tells us that $H\left[\vec{N}^{\uparrow}\right]$ does not change $T(x)$ but generates a displacement to the flow line whose Lagrange coordinates differ by the amount $N^{\uparrow k}(x)$ from the Lagrange coordinates $z^{k}=Z^{k}(x)$ of the flow line passing through $x$. This operation does not leave the hypersurface $Y(\Sigma)$ fixed; rather, it displaces each point of the hypersurface along the hypersurfaces of the dust foliation $\mathcal{T}$ (Fig. 3). Again, the Poisson bracket of any remaining variable with $H\left[\vec{N}^{\uparrow}\right]$ generates the change of this variable under such a displacement of the hypersurface.

The new form of the constraints leads to the familiar form of the Heisenberg equations of motion. Let $F\left[Z^{K}, P_{K}, g, p\right]$ be any dynamical variable on the phase space and choose a foliation $Z_{t}^{K}(x)=Z^{K}(t, x)$ of $\mathcal{T} \times \mathcal{S}$. The gravitational variables $g_{a b}(x)$ and $p^{a b}(x)$ evolve according to the Hamilton equations of motion from given initial data. These equations along with the Hamilton equations (4.14) and (4.15) determine $g_{a b}\left(x ; Z_{t}^{K}\right]$ and $p^{a b}\left(x ; Z_{t}^{K}\right]$; the constraints $(4.6),(4.9)$ then give $P_{K}\left(x ; Z_{t}^{K}\right]$. In this way the dynamical variable $F$ becomes a functional of $Z_{t}^{K}$,

$$
F\left[Z_{t}^{K}\right]:=F\left[Z_{t}^{K}, P_{K}\left[Z_{t}^{L}\right], g\left[Z_{t}^{L}\right], p\left[Z_{t}^{L}\right]\right] .
$$

The rate of change of this functional with $t$ is given by

$$
\dot{F}\left[Z_{t}^{K}\right]=\int_{\Sigma} d^{3} x \frac{d F\left[Z_{t}^{L}\right]}{\delta Z_{t}^{K}(x)} \dot{Z}_{t}^{K}(x)=\int_{\Sigma} d^{3} x \frac{d F\left[Z_{t}^{L}\right]}{\delta Z_{t}^{K}(x)} N_{t}^{\uparrow K}(x) .
$$

The symbol $d / \delta Z_{t}^{K}(x)$ denotes the total variational derivative of $F\left[Z_{t}^{K}\right]$ with respect to $Z_{t}^{K}(x)$, which takes into account both the explicit dependence of $F\left[Z^{K}, P_{K}, g, p\right]$ on $Z^{K}$ and the implicit dependence through the dynamical variables $g, p$, and $P_{K}$. On the other hand, $\dot{F}$ is also given by the Poisson bracket of $F\left[Z^{K}, P_{K}, g, p\right]$ with the Hamiltonian $H\left[N^{\uparrow K}\right]$. By comparing the two expressions for $\dot{F}$ for an arbitrary choice of foliation, we obtain the many-fingered time Heisenberg equations of motion

$$
\frac{d F}{\delta Z^{K}(x)}=\left\{F, H_{\uparrow K}(x)\right\}=\frac{\delta F}{\delta Z^{K}(x)}+\left\{F, h_{K}(x)\right\} .
$$

Here, $h_{K}(x)=\left(h(x), h_{k}(x)\right)$ are the true Hamiltonian and momentum densities from Eqs. (4.7) and (4.10). 


\section{Representing $\operatorname{LDiff}(\mathcal{T} \times \mathcal{S})$}

In Eqs. (4.12)-(4.13), the new constraints were smeared by externally prescribed multipliers $N^{\uparrow K}(x)$. The constraints also can be smeared by vector fields $\boldsymbol{V}_{1}, \boldsymbol{V}_{2}$ on $\mathcal{T} \times \mathcal{S}$ in the following way. Consider $\boldsymbol{V}_{1}$ and $\boldsymbol{V}_{2}$ as elements of $\operatorname{LDiff}(\mathcal{T} \times \mathcal{S})$ under the Lie bracket operation $-\left[\boldsymbol{V}_{1}, \boldsymbol{V}_{2}\right]$ which, up to a sign, is given by the commutator. When restricted to an embedding $Z^{K}(x)$, these vector fields become dynamical variables on the phase space of the dust. Thus, $\boldsymbol{V}_{1}$ and $\boldsymbol{V}_{2}$ can be used to smear the new constraints on $\Sigma$; for example,

$$
\boldsymbol{V}_{1} \mapsto H_{\uparrow}\left[\boldsymbol{V}_{1}\right]:=\int_{\Sigma} d^{3} x \boldsymbol{V}_{1}^{K}\left(Z^{L}(x)\right) H_{\uparrow K}(x)
$$

In this way each vector field on $\mathcal{T} \times \mathcal{S}$ is mapped into a dynamical variable over the phase space of dust coupled to gravity. Equations (4.6), (4.9), and (4.11) then guarantee that

$$
\left\{H_{\uparrow}\left[\boldsymbol{V}_{1}\right], H_{\uparrow}\left[\boldsymbol{V}_{2}\right]\right\}=H_{\uparrow}\left[-\left[\boldsymbol{V}_{1}, \boldsymbol{V}_{2}\right]\right]
$$

i.e., that the mapping (4.19) is a homomorphism from $\operatorname{LDiff}(\mathcal{T} \times \mathcal{S})$ into the Poisson bracket algebra over the phase space. In this manner, the introduction of dust enables us to represent the Lie algebra of the dust spacetime diffeomorphism group by canonical transformations.

\section{New Constraints Lead to a True Algebra for Vacuum Gravity}

The new Hamiltonian constraint (4.6) has a feature that is quite astounding: The Hamiltonian $h(x)$ of Eq. (4.7) does not depend on the dust variables, but only on the geometric variables $g_{a b}, p^{a b}$. We have not encountered such behavior in any other physical system. Even for the simplest parametrized systems, like a massless scalar field on a Minkowskian background [23], the Hamiltonian depends on the many-fingered Minkowski time. In contrast, the Hamiltonian (4.7) turns out to be independent of the time $T(x)$ and frame $Z^{k}(x)$ variables.

This remarkable feature has an equally remarkable consequence: The Poisson brackets $\left\{H_{\uparrow}(x), H_{\uparrow}\left(x^{\prime}\right)\right\}=0$ do not have any cross terms between $P(x)$ and $h(x)$, and hence $h(x)$ must have vanishing Poisson brackets among themselves:

$$
\left\{h(x ; g, p], h\left(x^{\prime} ; g, p\right]\right\}=0 .
$$

Since the Hamiltonian $h(x)$ is a square root (4.7), it follows that the expressions $G(x)$ under the square root must also have vanishing Poisson brackets:

$$
\left\{G(x ; g, p], G\left(x^{\prime} ; g, p\right]\right\}=0 .
$$

This property is vital for the definition of the Hamiltonian operator $h(x ; \hat{g}, \hat{p}]$ by spectral analysis (Section 6c).

We proved Eq. (4.22) by coupling geometry to dust. However, Eq. (4.22) is an identity involving only the geometric variables $g_{a b}$ and $p^{a b}$. Thus, it must hold irrespective of whether geometry is coupled to dust, to any other matter system, or whether it is left alone in vacuum. Equation (4.22) must simply hold by virtue of the Dirac 'algebra' (4.1)-(4.3) among the 
gravitational expressions $H_{\perp}^{G}(x)$ and $H_{a}^{G}(x)$. We verify this assertion directly in Appendix E.

We have found a simple quadratic combination $G(x)$ of the gravitational superHamiltonian $H_{\perp}^{G}(x)$ and supermomentum $H_{a}^{G}(x)$ that strongly commutes with itself, Eq. (4.22). Because $G(x)$ is a scalar density of weight 2 constructed solely from the geometric variables, its Poisson bracket with the gravitational supermomentum is given by

$$
\left\{G(x), H_{a}^{G}\left(x^{\prime}\right)\right\}=G_{, a}(x) \delta\left(x, x^{\prime}\right)+2 G(x) \delta_{, a}\left(x, x^{\prime}\right) .
$$

Of course, the gravitational supermomenta represent LDiff $\Sigma$,

$$
\left\{H_{a}^{G}(x), H_{b}^{G}\left(x^{\prime}\right)\right\}=H_{b}^{G}(x) \delta_{, a}\left(x, x^{\prime}\right)-\left(a x \leftrightarrow b x^{\prime}\right) .
$$

Thus, the geometric expressions $G(x)$ and $H_{a}^{G}(x)$ form a true Lie algebra that is a semidirect product of the Abelian algebra generated by $G(x)$ and LDiff $\Sigma$ generated by $H_{a}^{G}(x)$.

In vacuum gravity, the imposition of the usual super-Hamiltonian and supermomentum constraints $H_{\perp}^{G}(x)$ and $H_{a}^{G}(x)$ is clearly globally equivalent to the imposition of the new set of constraints, $G(x)$ and $H_{a}^{G}(x)$ :

$$
H_{\perp}^{G}(x)=0=H_{a}^{G}(x) \Longleftrightarrow G(x)=0=H_{a}^{G}(x) .
$$

Therefore, we have succeeded in casting vacuum gravity into a form in which the constraints close according to a true Lie algebra. This is something that previously has been achieved only for systems in two-dimensional spacetimes, such as strings [24 and 2-dimensional gravity 25.

There is only one fly in the ointment. In vacuum gravity, the Hamiltonian vector field generated by $G(x)$ vanishes on the constraint surface (4.25). Therefore, $G(x)$ does not generate a motion on the constraint surface, and it cannot be used for foliating the constraint surface into orbits. Such a difficulty does not exist for gravity coupled to dust: As long as there is any dust at $x$,

$$
G(x)=P^{2}(x)>0
$$

The full significance of the new form of the constraints for vacuum gravity remains to be investigated.

\section{CANONICAL DUST-SPACE VARIABLES}

\section{A. The Dust-Space Variables and the Constraints}

Our description of dust coupled to gravity was based on the conjugate pairs $Z^{k}(x)$ and $P_{k}(x), T(x)$ and $P(x)$, and $g_{a b}(x)$ and $p^{a b}(x)$ of canonical variables. The variables $T(x)$, $P(x), g_{a b}(x)$, and $p^{a b}(x)$ are (t-dependent) tensor fields on $\Sigma$. The variable $Z^{k}(x)$ is the local coordinate chart expression of a ( $t$-dependent) mapping $Z: \Sigma \rightarrow \mathcal{S}$ from the space manifold $\Sigma$ to the dust space $\mathcal{S}$. Its conjugate $P_{k}(x)$ is the coordinate expression of a ( $t$-dependent) mapping from $\Sigma$ to $T^{*} \mathcal{S}$, with density weight 1 on $\Sigma$. The Hamiltonian formulation of 
the combined system is characterized by the constraints $H_{\uparrow}(x)=0$ and $H_{\uparrow k}(x)=0$ which generate the abelian algebra (4.11).

Canonical quantization is vastly simplified by the introduction of an alternative set of canonical variables which reflect the fact that the dust particles naturally define a preferred set of spatial coordinates on $\Sigma$. In this section, we construct such variables, and explain how they are geared to the abelian algebra (4.11).

Start from the tensorial variables $T(x), P(x), g_{a b}(x)$, and $p^{a b}(x)$ on $\Sigma$, and let the mapping $Z: \Sigma \rightarrow \mathcal{S}$ induce a corresponding set of tensors $\boldsymbol{T}(z), \boldsymbol{P}(z), \boldsymbol{g}_{k \ell}(z)$, and $\boldsymbol{p}^{k \ell}(z)$ on the dust space $\mathcal{S}$. In terms of local coordinates, these $\mathcal{S}$-variables are defined by

$$
\begin{aligned}
\boldsymbol{T}(z) & :=T(X(z)) \\
\boldsymbol{P}(z) & :=\left|\frac{\partial X(z)}{\partial z}\right| P(X(z)), \\
\boldsymbol{g}_{k \ell}(z) & :=X_{, k}^{a}(z) X_{, \ell}^{b}(z) g_{a b}(X(z)), \\
\boldsymbol{p}^{k \ell}(z) & :=\left|\frac{\partial X(z)}{\partial z}\right| Z_{, a}^{k}(X(z)) Z_{, b}^{\ell}(X(z)) p^{a b}(X(z)) .
\end{aligned}
$$

Here, the $t$-dependent mapping $X$ is simply the inverse of $Z$,

$$
X: \mathcal{S} \rightarrow \Sigma, \quad X:=Z^{-1},
$$

and $|\partial X(z) / \partial z|$ is the Jacobian for the change of variables $x^{a}=X^{a}(z)$.

The $\mathcal{S}$-variable $\boldsymbol{T}(z)$ measures proper time along the dust worldline whose Lagrangian coordinate label is $z^{k}$, and $\boldsymbol{P}(z)$ is the dust rest mass on $\Sigma$ per unit coordinate cell $d^{3} z$. The variables $\boldsymbol{g}_{k \ell}(z)$ are the components of the metric tensor

$$
d s^{2}=\boldsymbol{g}_{k \ell}(z) d z^{k} d z^{\ell}
$$

that measures proper distance $d s$ in $\Sigma$ between neighboring dust particles with Lagrangian coordinate labels $z^{k}$ and $z^{k}+d z^{k}$.

We will now show that the $\mathcal{S}$-variables $\boldsymbol{T}(z), \boldsymbol{P}(z), \boldsymbol{g}_{k \ell}(z)$, and $\boldsymbol{p}^{k \ell}(z)$, along with the dust frame $Z^{k}(x)$ and the new supermomentum $H_{\uparrow k}(x)$, are canonically conjugate. That is, we will argue that the only nonvanishing Poisson brackets among these variables are

$$
\begin{aligned}
\left\{Z^{k}(x), H_{\uparrow \ell}\left(x^{\prime}\right)\right\} & =\delta_{\ell}^{k} \delta\left(x, x^{\prime}\right), \\
\left\{\boldsymbol{T}(z), \boldsymbol{P}\left(z^{\prime}\right)\right\} & =\delta\left(z, z^{\prime}\right), \\
\left\{\boldsymbol{g}_{k \ell}(z), \boldsymbol{p}^{m n}\left(z^{\prime}\right)\right\} & =\frac{1}{2}\left(\delta_{k}^{m} \delta_{\ell}^{n}+\delta_{\ell}^{m} \delta_{k}^{n}\right) \delta\left(z, z^{\prime}\right) .
\end{aligned}
$$

Recall (Section 4b) that the constraint functions $H_{\uparrow k}(x)$ have strongly vanishing Poisson brackets among themselves. They are thus natural candidates for a new set of canonical momenta. Equation (5.4a) maintains that their conjugate coordinates are the frame variables $Z^{k}(x)$. This is obvious from the construction (4.9) of $H_{\uparrow k}(x)$.

The Poisson brackets (5.4b) and (5.4c) exemplify a general rule: the mapping $\Sigma \rightarrow \mathcal{S}$ turns canonically conjugate fields on $\Sigma$ into canonically conjugate fields on $\mathcal{S}$. This is most 
easily established for a scalar field and its conjugate, such as the proper time (5.1a) and the rest mass density (5.1b). In this case, we first write

$$
\begin{aligned}
& \boldsymbol{T}(z)=\int_{\Sigma} d^{3} x T(x) \delta(x-X(z)), \\
& \boldsymbol{P}(z)=\int_{\Sigma} d^{3} x P(x) \delta(Z(x)-z),
\end{aligned}
$$

and then directly verify that the Poisson brackets

$$
\left\{\boldsymbol{T}(z), \boldsymbol{P}\left(z^{\prime}\right)\right\}=\int_{\Sigma} d^{3} x\left(\frac{\delta \boldsymbol{T}(z)}{\delta T(x)} \frac{\delta \boldsymbol{P}\left(z^{\prime}\right)}{\delta P(x)}-\frac{\delta \boldsymbol{T}(z)}{\delta P(x)} \frac{\delta \boldsymbol{P}\left(z^{\prime}\right)}{\delta T(x)}\right)+\cdots
$$

yield the desired result (5.4b). (The unwritten terms in Eq. (5.6) involve functional derivatives with respect to $Z^{k}(x), P_{k}(x), g_{a b}(x)$, and $p^{a b}(x)$, and they all vanish.) It is also clear that the Poisson brackets of $T(z)$ among themselves and of $P(z)$ among themselves strongly vanish. It is straightforward to extend these arguments to arbitrary canonically conjugate tensorial variables and, in particular, to show that $\boldsymbol{g}_{k \ell}(z)$ and $\boldsymbol{p}^{k \ell}(z)$ are canonically conjugate.

The $\mathcal{S}$-variables $\boldsymbol{T}(z), \boldsymbol{P}(z), \boldsymbol{g}_{k \ell}(z)$, and $\boldsymbol{p}^{k \ell}(z)$ obviously have vanishing Poisson brackets with the frame variables $Z^{k}(x)$. The only remaining task is to show that their brackets with the new momentum $H_{\uparrow k}(x)$ also vanish. To do that, let us recall the relationship $H_{\uparrow k}(x)=Z_{k}^{a}(x) H_{a}(x)$ between the new supermomentum $H_{\uparrow k}(x)$ and the original supermomentum $H_{a}(x)$. Since the matrix $Z_{k}^{a}$ depends only on the frame variables $Z^{k}(x)$ and the $\mathcal{S}$-variables (5.1) are independent of $P_{k}(x)$, they have vanishing brackets with $Z_{k}^{a}(x)$. Thus, the Poisson brackets of the $\mathcal{S}$-variables with $H_{\uparrow k}(x)$ are proportional to their brackets with $H_{a}(x)$. However, the Poisson brackets of the $\mathcal{S}$-variables with $H_{a}(x)$ must vanish. Recall that the Poisson brackets of any dynamical variable with the supermomentum smeared by an externally prescribed vector field $N^{a}(x)$ on $\Sigma$,

$$
H[\vec{N}]:=\int_{\Sigma} d^{3} x N^{a}(x) H_{a}(x),
$$

give the change in that variable under LDiff $\Sigma$. Since the $\mathcal{S}$-variables are constructed so that they are invariant under Diff $\Sigma$, their Poisson brackets with $H[\vec{N}]$ and hence with $H_{a}(x)$ vanish. It follows that their brackets with $H_{\uparrow k}(x)$ also vanish. This completes our proof that the $\mathcal{S}$-variables (5.1) along with the pair $Z^{k}(x), H_{\uparrow k}(x)$ form a canonical chart.

In terms of the new canonical variables $\boldsymbol{T}(z), \boldsymbol{P}(z), \boldsymbol{g}_{k \ell}(z), \boldsymbol{p}^{k \ell}(z)$, and $Z^{k}(x), H_{\uparrow k}(x)$, the supermomentum constraint is simply the condition that the canonical momentum $H_{\uparrow k}(x)$ vanishes. The super-Hamiltonian constraint $H_{\uparrow}(x)=0$ can then be mapped to the dust space $\mathcal{S}$, which yields an equivalent constraint

$$
\boldsymbol{H}_{\uparrow}(z):=\left|\frac{\partial X(z)}{\partial z}\right| H_{\uparrow}(X(z))=\boldsymbol{P}(z)+\boldsymbol{h}\left(z ; \boldsymbol{g}_{k \ell}, \boldsymbol{p}^{k \ell}\right]=0 .
$$

Here, the Hamiltonian density $\boldsymbol{h}(z)=-\sqrt{\boldsymbol{G}(z)}$ is obtained from $h(x)$ of Eq. (4.7) by replacing the $\Sigma$-tensors $g_{a b}(x)$ and $p^{a b}(x)$ with the corresponding $\mathcal{S}$-tensors $\boldsymbol{g}_{k \ell}(z)$ and $\boldsymbol{p}^{k \ell}(z)$. The constraint $\boldsymbol{H}_{\uparrow}(z)$ is expressed entirely in terms of the $\mathcal{S}$-variables. Its Poisson brackets with $H_{\uparrow k}(x)$ therefore vanish. We conclude that the constraints $\boldsymbol{H}_{\uparrow}(z)$ and $H_{\uparrow k}(x)$ still form an abelian algebra. 


\section{B. Noether Charges}

The Noether charge $Q[\boldsymbol{\vartheta}]$ from Eq. (3.25) can be expressed in terms of the new canonical variables by a simple change $x^{a}=X^{a}(z)$ of integration variables. This gives

$$
\begin{aligned}
Q[\boldsymbol{\vartheta}] & =\int_{\Sigma} d^{3} x \boldsymbol{\vartheta}(Z(x)) P(x) \\
& =\int_{\mathcal{S}} d^{3} z \boldsymbol{\vartheta}(z) \boldsymbol{P}(z)
\end{aligned}
$$

As discussed in Secs. 3b and 2e, $Q[\boldsymbol{\vartheta}]$ generates a canonical transformation that yields the change of any dynamical variable induced by the change of the initial hypersurface of constant dust time $T(y)=\tau=$ const. Among the new canonical variables, only the dust proper time $T(z)$ is affected by $Q[\boldsymbol{\vartheta}]$ :

$$
\{\boldsymbol{T}(z), Q[\boldsymbol{\vartheta}]\}=\boldsymbol{\vartheta}(z)
$$

The canonical transformation generated by $Q[\boldsymbol{\vartheta}]$ produces a translation of the proper time $\tau$ along the dust worldline $z^{k}$ by the amount $\boldsymbol{\vartheta}(z)$.

The expressions (4.6)-(4.10) can be used to write the Noether charge $Q[\overrightarrow{\boldsymbol{\xi}}]$ from Eq. (3.24) in terms of the new canonical variables. The result is

$$
\begin{aligned}
Q[\overrightarrow{\boldsymbol{\xi}}] & =-\int_{\Sigma} d^{3} x \boldsymbol{\xi}^{k}(Z(x)) P_{k}(x) \\
& =-\int_{\Sigma} d^{3} x \boldsymbol{\xi}^{k}(Z(x)) H_{\uparrow k}(x)+\int_{\mathcal{S}} d^{3} z \boldsymbol{\xi}^{k}(z)\left(\boldsymbol{H}_{k}^{G}(z)+\boldsymbol{P}(z) \boldsymbol{T}_{, k}(z)\right),
\end{aligned}
$$

where $\boldsymbol{H}_{k}^{G}(z)$ is obtained by mapping the gravitational supermomentum $H_{a}^{G}(x)$ from space $\Sigma$ to the dust space $\mathcal{S}$ :

$$
\boldsymbol{H}_{k}^{G}(z)=\left|\frac{\partial X(z)}{\partial z}\right| X_{, k}^{a}(z) H_{a}^{G}(X(z)) .
$$

Thus, $\boldsymbol{H}_{k}^{G}(z)$ depends only on $\boldsymbol{g}_{k \ell}(z)$ and $\boldsymbol{p}^{k \ell}(z)$.

The Noether charge $Q[\overrightarrow{\boldsymbol{\xi}}]$ can be recognized as the canonical generator of dust space diffeomorphisms, Diff $\mathcal{S}$. That is, for any canonical variable $F$, the Poisson brackets $\{F, Q[\overrightarrow{\boldsymbol{\xi}}]\}$ give the change in $F$ due to an infinitesimal diffeomorphism of $\mathcal{S}$ generated by the vector field $\overrightarrow{\boldsymbol{\xi}}(z)$. If $F$ is one of the $\mathcal{S}$-tensors $\boldsymbol{T}, \boldsymbol{P}, \boldsymbol{g}$, or $\boldsymbol{p}$, then the Poisson brackets $\{F, Q[\overrightarrow{\boldsymbol{\xi}}]\}$ equal the Lie derivative $£_{\vec{\xi}} F$. For the dust frame variable $Z^{k}(x)$ and its conjugate $H_{\uparrow k}(x)$, the transformation generated by $Q[\overrightarrow{\boldsymbol{\xi}}]$ is

$$
\begin{aligned}
\left\{Z^{k}(x), Q[\overrightarrow{\boldsymbol{\xi}}]\right\} & =-\boldsymbol{\xi}^{k}(Z(x)), \\
\left\{H_{\uparrow k}(x), Q[\overrightarrow{\boldsymbol{\xi}}]\right\} & =\boldsymbol{\xi}_{, k}^{\ell}(Z(x)) H_{\uparrow \ell}(x) .
\end{aligned}
$$

The charges $Q[\boldsymbol{\vartheta}]$ and $Q[\overrightarrow{\boldsymbol{\xi}}]$ close under the Poisson brackets according to the relations (3.28).

As noted in Sec. 3b, the Noether charges $Q[\boldsymbol{\vartheta}]$ and $Q[\overrightarrow{\boldsymbol{\xi}}]$ have vanishing Poisson brackets with the constraints $H_{\perp}(x)$ and $H_{a}(x)$. Since the new set of constraints $H_{\uparrow k}(x)=0$ and 
$\boldsymbol{H}_{\uparrow}(z)=0$ are equivalent to the old set, the Poisson brackets of the charges with the new constraints should vanish at least weakly, that is, when the constraints hold. Indeed, the Poisson brackets of $Q[\boldsymbol{\vartheta}]$ with $H_{\uparrow k}(x)$ and $\boldsymbol{H}_{\uparrow}(z)$ vanish strongly. The Poisson brackets of $Q[\overrightarrow{\boldsymbol{\xi}}]$ with $H_{\uparrow k}(x)$ vanish when the constraints hold, as seen from Eq. (5.13b). The remaining Poisson brackets are

$$
\left\{\boldsymbol{H}_{\uparrow}(z), Q[\overrightarrow{\boldsymbol{\xi}}]\right\}=\left(\boldsymbol{H}_{\uparrow}(z) \boldsymbol{\xi}^{k}(z)\right)_{, k}
$$

and they also weakly vanish. Expression (5.14) shows that $\boldsymbol{H}_{\uparrow}(z)$ transforms as a scalar density under Diff $\mathcal{S}$.

\section{QUANTIZATION}

\section{A. Operator Constraints and the Functional Schrödinger Equation}

In the Dirac method of quantization, constraints are turned into operators and imposed as restrictions on the state functionals of the system. With the standard form (3.20) of the super-Hamiltonian constraints, this procedure leads to the Wheeler-DeWitt equation for gravity coupled to a source. This is a second-order variational differential equation in the metric variables whose solution space carries no obvious Hilbert space structure. If the source happens to be dust, there is an additional difficulty: the square-root Hamiltonian density (3.19) is difficult to define by spectral analysis because the expressions under the square root do not commute, Eq. (4.5).

In order for dust to serve as a clock, the super-Hamiltonian constraint should be resolved with respect to the momentum conjugate to the dust time variable prior to quantization. The constraint $H_{\uparrow}(x)$, or equivalently $\boldsymbol{H}_{\uparrow}(z)$, has this desired form. We choose to work primarily with the dust-space variables, so the quantum states of the system are functionals $\boldsymbol{\Psi}[Z, \boldsymbol{T}, \boldsymbol{g}]$ of the canonical coordinates $Z^{k}(x), \boldsymbol{T}(z)$, and $\boldsymbol{g}_{k \ell}(z)$. From the expression (5.8) of the classical constraint $\boldsymbol{H}_{\uparrow}(z)=0$, the operator condition

$$
\boldsymbol{H}_{\uparrow}(z ; \hat{\boldsymbol{P}}, \hat{\boldsymbol{g}}, \hat{\boldsymbol{p}}] \boldsymbol{\Psi}[Z, \boldsymbol{T}, \boldsymbol{g}]=0
$$

yields the functional Schrödinger equation

$$
i \frac{\delta \boldsymbol{\Psi}[Z, \boldsymbol{T}, \boldsymbol{g}]}{\delta \boldsymbol{T}(z)}=\boldsymbol{h}(z ; \hat{\boldsymbol{g}}, \hat{\boldsymbol{p}}] \boldsymbol{\Psi}[Z, \boldsymbol{T}, \boldsymbol{g}]
$$

In addition to Eq. (6.2), the state functionals must obey the operator version of the classical constraint $H_{\uparrow k}(x)=0$, namely,

$$
\hat{H}_{\uparrow k}(x) \boldsymbol{\Psi}[Z, \boldsymbol{T}, \boldsymbol{g}]=0
$$

Because $H_{\uparrow k}(x)$ is canonically conjugate to $Z^{k}(x), \hat{H}_{\uparrow k}(x)$ is the momentum operator

$$
\hat{H}_{\uparrow k}(x)=-i \frac{\delta}{\delta Z^{k}(x)} .
$$


Equation (6.3) then implies that the quantum states must be independent of the dust frame $Z^{k}(x)$. Therefore the state functionals $\boldsymbol{\Psi}[\boldsymbol{T}, \boldsymbol{g}]$ satisfy the functional Schrödinger equation

$$
i \frac{\delta \boldsymbol{\Psi}[\boldsymbol{T}, \boldsymbol{g}]}{\delta \boldsymbol{T}(z)}=\boldsymbol{h}(z ; \hat{\boldsymbol{g}}, \hat{\boldsymbol{p}}] \boldsymbol{\Psi}[\boldsymbol{T}, \boldsymbol{g}]
$$

with no further restrictions.

The functional differential equations (6.1), (6.3) are consistent only if the commutators of the operators $\hat{\boldsymbol{H}}_{\uparrow}(z)$ and $\hat{H}_{\uparrow k}(x)$ replicate the classical Poisson bracket algebra

$$
\begin{aligned}
\left\{\boldsymbol{H}_{\uparrow}(z), \boldsymbol{H}_{\uparrow}\left(z^{\prime}\right)\right\} & =0, \\
\left\{\boldsymbol{H}_{\uparrow}(z), H_{\uparrow k}(x)\right\} & =0, \\
\left\{H_{\uparrow k}(x), H_{\uparrow \ell}\left(x^{\prime}\right)\right\} & =0 .
\end{aligned}
$$

With the representation (6.4) for the momentum operator the subalgebra (6.6c) is indeed carried over into the quantum theory. The brackets $(6.6 \mathrm{~b})$ are also satisfied quantum mechanically since each of the operators $\hat{\boldsymbol{P}}, \hat{\boldsymbol{g}}, \hat{\boldsymbol{p}}$ that appear in $\hat{\boldsymbol{H}}_{\uparrow}(z)$ commutes with $\hat{H}_{\uparrow k}(x)$. We shall proceed under the assumption that there exists a factor ordering and regularization of $\boldsymbol{h}(z ; \hat{\boldsymbol{g}}, \hat{\boldsymbol{p}}]$ such that the quantum operators $\hat{\boldsymbol{H}}_{\uparrow}(z)$ commute among themselves, and thereby replicate the vanishing of the classical Poisson brackets (6.6a).

\section{B. Time-Independent Schrödinger Equation}

$\hat{\boldsymbol{H}}_{\uparrow}(z)$ does not depend on $\boldsymbol{T}(z)$ so that

$$
\left[\hat{\boldsymbol{H}}_{\uparrow}(z), \hat{\boldsymbol{P}}\left(z^{\prime}\right)\right]=0 .
$$

Furthermore, the operators $\hat{\boldsymbol{P}}(z)$ commute among themselves, and the super-Hamiltonian operators $\hat{\boldsymbol{H}}_{\uparrow}(z)$ commute by virtue of Eq. (6.6a). It follows that the Schrödinger equation (6.5) and the eigenvalue equation

$$
\hat{\boldsymbol{P}}(z) \boldsymbol{\Psi}=\boldsymbol{P}(z) \boldsymbol{\Psi}
$$

have common solutions $\boldsymbol{\Psi}[\boldsymbol{P} ; \boldsymbol{T}, \boldsymbol{g}]$. These are functionals of $\boldsymbol{T}(z)$ and $\boldsymbol{g}_{k \ell}(z)$ labeled by the eigenvalues $\boldsymbol{P}(z)$ of the momentum operator $\hat{\boldsymbol{P}}(z)$. The general solution of Eq. (6.8) has the form

$$
\boldsymbol{\Psi}[\boldsymbol{P} ; \boldsymbol{T}, \boldsymbol{g}]=\boldsymbol{\psi}[\boldsymbol{g}] \exp \left(i \int_{\mathcal{S}} d^{3} z \boldsymbol{P}(z) \boldsymbol{T}(z)\right)
$$

By substituting expression (6.9) into Eq. (6.5) we learn that $\boldsymbol{\psi}[\boldsymbol{g}]$ must satisfy the timeindependent functional Schrödinger equation

$$
\boldsymbol{h}(z ; \hat{\boldsymbol{g}}, \hat{\boldsymbol{p}}] \boldsymbol{\psi}[\boldsymbol{g}]=-\boldsymbol{P}(z) \boldsymbol{\psi}[\boldsymbol{g}]
$$

Equations (6.9) and (6.10) accomplish the separation of the many-fingered time $\boldsymbol{T}(z)$ from the metric $\boldsymbol{g}_{k \ell}(z)$. 


\section{Spectral Analysis}

The classical Hamiltonian density $\boldsymbol{h}(z)$ is the dust space counterpart of the square root Hamiltonian density $h(x)=-\sqrt{G(x)}$, as defined in Eq. (4.7). The square root structure of $h(x)$ is inherited by $\boldsymbol{h}(z)$, so that $\boldsymbol{h}(z)=-\sqrt{\boldsymbol{G}(z)}$ where

$$
\boldsymbol{G}(z ; \boldsymbol{g}, \boldsymbol{p}]:=\left(\boldsymbol{H}_{\perp}^{G}(z)\right)^{2}-\boldsymbol{g}^{k \ell}(z) \boldsymbol{H}_{k}^{G}(z) \boldsymbol{H}_{\ell}^{G}(z) .
$$

As a consequence, the operators $\hat{\boldsymbol{h}}(z)$ that appear in the functional Schrödinger equation must be defined by spectral analysis. The first step is to turn the classical expressions (6.11) into operators $\boldsymbol{G}(z ; \hat{\boldsymbol{g}}, \hat{\boldsymbol{p}}]$. The assumption that the operators $\hat{\boldsymbol{H}}_{\uparrow}(z)$ commute translates into the assumption that there exists a factor ordering of $\hat{\boldsymbol{g}}$ and $\hat{\boldsymbol{p}}$, and a regularization of $\hat{\boldsymbol{G}}(z)$, such that

$$
\left[\boldsymbol{G}(z ; \hat{\boldsymbol{g}}, \hat{\boldsymbol{p}}], \boldsymbol{G}\left(z^{\prime} ; \hat{\boldsymbol{g}}, \hat{\boldsymbol{p}}\right]\right]=0
$$

The operators $\hat{\boldsymbol{G}}(z)$ then have common eigenfunctionals $\phi[\boldsymbol{G} ; \boldsymbol{g}]$,

$$
\hat{\boldsymbol{G}}(z) \phi[\boldsymbol{G} ; \boldsymbol{g}]=\boldsymbol{G}(z) \phi[\boldsymbol{G} ; \boldsymbol{g}],
$$

with eigenvalues $\boldsymbol{G}(z)$.

The classical variables (6.11) are not positive definite, so the eigenvalues $\boldsymbol{G}(z)$ are not necessarily positive everywhere on $\mathcal{S}$. Let us define a Hilbert space $\mathcal{H}^{+}$that is spanned by the eigenfunctionals of $\boldsymbol{G}(z)$ with positive eigenvalues $\boldsymbol{G}(z)>0$. On $\mathcal{H}^{+}$, we can define the operator

$$
\hat{\boldsymbol{h}}(z)=-\sqrt{\hat{\boldsymbol{G}}(z)}
$$

by the requirement that it have eigenfunctionals $\boldsymbol{\phi}[\boldsymbol{G} ; \boldsymbol{g}]$ with eigenvalues

$$
\boldsymbol{h}(z)=-\sqrt{\boldsymbol{G}(z)} .
$$

Because $\hat{\boldsymbol{h}}(z)$ is the Hamiltonian of the system, a state functional in $\mathcal{H}^{+}$will remain in $\mathcal{H}^{+}$ throughout its dynamical evolution.

If an eigenfunctional $\boldsymbol{\phi}[\boldsymbol{G} ; \boldsymbol{g}]$ with eigenvalue $\boldsymbol{G}(z)$ is known, then infinitely many eigenfunctionals corresponding to other eigenvalues can be generated by the action of dust space diffeomorphisms $\boldsymbol{\Xi} \in \operatorname{Diff} \mathcal{S}$. This result is expressed by the following theorem, which is proved in Appendix G: Let $\phi[\boldsymbol{G} ; \boldsymbol{g}]$ be an eigenfunctional of $\hat{\boldsymbol{G}}(z)$ with eigenvalue $\boldsymbol{G}(z)$. Then $\boldsymbol{\phi}\left[\boldsymbol{G} ; \boldsymbol{\Xi}^{*} \boldsymbol{g}\right]$ is an eigenfunctional of $\hat{\boldsymbol{G}}(z)$ with eigenvalue $\left(\boldsymbol{\Xi}^{-1}\right)^{*} \boldsymbol{G}(z)$.

Disregarding global difficulties, a given dust space scalar density $\boldsymbol{G}(z)>0$ of weight 2 can be transformed by the action of an appropriate diffeomorphism $\boldsymbol{\Xi}^{-1}$ into any other arbitrary function $\boldsymbol{G}_{*}(z)>0$. In particular, it can be transformed into $\boldsymbol{G}_{*}(z)=1$. This means that $\hat{\boldsymbol{G}}(z)$ has a continuous spectrum, i.e., that there is an eigenfunctional $\boldsymbol{\phi}[\boldsymbol{G} ; \boldsymbol{g}]$ for any $\boldsymbol{G}(z)>0$. 
These arguments also indicate that the spectrum of the collection $z \in \mathcal{S}$ of commuting operators $\hat{\boldsymbol{G}}(z)$ is infinitely degenerate. Indeed, let $\boldsymbol{\Xi}_{0} \in \operatorname{Diff}_{0} \mathcal{S}$ be an arbitrary volumepreserving diffeomorphism, $\operatorname{det}\left(\partial \boldsymbol{\Xi}_{0}(z) / \partial z\right)=1$. For $\boldsymbol{G}(z)=1$, we have also $\left(\boldsymbol{\Xi}_{0}^{-1}\right)^{*} \boldsymbol{G}(z)=$ 1. Therefore, according to the theorem stated above, if $\boldsymbol{\phi}[1 ; \boldsymbol{g}]$ is an eigenfunctional of $\hat{\boldsymbol{G}}(z)$ with eigenvalue 1 , then $\boldsymbol{\phi}\left[1 ; \boldsymbol{\Xi}_{0}^{*} \boldsymbol{g}\right]$ is another eigenfunctional of $\hat{\boldsymbol{G}}(z)$ with eigenvalue 1. This shows that there are at least as many eigenfunctionals of $\hat{\boldsymbol{G}}(z)$ with eigenvalue 1 as there are volume-preserving diffeomorphisms. When we act on the argument of this set of eigenfunctionals by an arbitrary diffeomorphism $\boldsymbol{\Xi}$, we turn it into a set of eigenfunctionals

$$
\boldsymbol{\phi}\left[1 ; \boldsymbol{\Xi}^{*} \boldsymbol{\Xi}_{0}^{*} \boldsymbol{g}\right], \quad \boldsymbol{\Xi}_{0} \in \operatorname{Diff}_{0} \mathcal{S}
$$

where each eigenfunctional has the eigenvalue $\boldsymbol{G}(z):=(\operatorname{det}(\partial \boldsymbol{\Xi}(z) / \partial z))^{-2}$. In this way, we conclude that an arbitrary level $\boldsymbol{G}(z)>0$ is infinitely degenerate.

It remains an open question whether there is more degeneracy of the $\boldsymbol{G}(z)>0$ levels than that corresponding to the action of volume-preserving diffeomorphisms, and, if so, how the additional degeneracy should be labeled. We do not need to answer this question. Formally, the degeneracy of the levels $\boldsymbol{G}(z)>0$ can be taken into account by the introduction of a degeneracy index $d$. The Hilbert space $\mathcal{H}^{+}$is spanned by the eigenfunctionals $\boldsymbol{\phi}_{d}[\boldsymbol{G} ; \boldsymbol{g}]$ which satisfy

$$
\hat{\boldsymbol{G}}(z) \boldsymbol{\phi}_{d}[\boldsymbol{G} ; \boldsymbol{g}]=\boldsymbol{G}(z) \boldsymbol{\phi}_{d}[\boldsymbol{G} ; \boldsymbol{g}] \text {. }
$$

An arbitrary state $\boldsymbol{\Psi}[\boldsymbol{g}]$ in $\mathcal{H}^{+}$can be written as a linear superposition

$$
\boldsymbol{\Psi}[\boldsymbol{g}]=\sum_{d} \int_{G(z)>0} \mathrm{D} \boldsymbol{G} C_{d}[\boldsymbol{G}] \boldsymbol{\phi}_{d}[\boldsymbol{G} ; \boldsymbol{g}]
$$

of the eigenfunctionals $\boldsymbol{\phi}_{d}[\boldsymbol{G} ; \boldsymbol{g}]$ with coefficients $C_{d}[\boldsymbol{G}]$. These eigenfunctionals form a basis for the energy-density representation (6.14)-(6.15).

From the considerations above, the general solution of the functional Schrödinger equation (6.5) can be written in the form

$$
\boldsymbol{\Psi}[\boldsymbol{T}, \boldsymbol{g}]=\boldsymbol{U}\left[\boldsymbol{T}-\boldsymbol{T}_{0} ; \hat{\boldsymbol{g}}, \hat{\boldsymbol{p}}\right] \boldsymbol{\Psi}\left[\boldsymbol{T}_{0}, \boldsymbol{g}\right]
$$

where

$$
\boldsymbol{U}\left[\boldsymbol{T}-\boldsymbol{T}_{0} ; \hat{\boldsymbol{g}}, \hat{\boldsymbol{p}}\right]:=\exp \left(-i \int_{\mathcal{S}} d^{3} z\left(\boldsymbol{T}(z)-\boldsymbol{T}_{0}(z)\right) \hat{\boldsymbol{h}}(z)\right)
$$

Here, $\boldsymbol{\Psi}\left[\boldsymbol{T}_{0}, \boldsymbol{g}\right]$ is an arbitrary initial state in $\mathcal{H}^{+}$, and the operator $\hat{\boldsymbol{h}}(z)$ is defined by spectral analysis. The state $\boldsymbol{\Psi}[\boldsymbol{T}, \boldsymbol{g}]$ at the dust time $\boldsymbol{T}(z)$ follows from the initial state $\boldsymbol{\Psi}\left[\boldsymbol{T}_{0}, \boldsymbol{g}\right]$ at the dust time $\boldsymbol{T}_{0}(z)$ by the action of the many-fingered-time evolution operator $\boldsymbol{U}\left[\boldsymbol{T}-\boldsymbol{T}_{0} ; \hat{\boldsymbol{g}}, \hat{\boldsymbol{p}}\right]$.

\section{The Action of Symmetries on States}

The wave functional $\boldsymbol{\Psi}[Z, \boldsymbol{T}, \boldsymbol{g}]$ characterizes the quantum state of the gravitational field on the hypersurface specified by the dust time $\boldsymbol{T}(z)$. Such a description is not unique. First 
of all, the dust time is not unique because the time foliation $\mathcal{T}$ depends on the choice of the fiducial hypersurface $\boldsymbol{T}(z)=0$. By changing this hypersurface, the time variable $\boldsymbol{T}(z)$ is changed according to

$$
\boldsymbol{T}^{\prime}(z)=\boldsymbol{T}(z)+\Theta(z) .
$$

(This is Eq. (2.27) expressed in the dust coordinates.) The variables $\boldsymbol{T}(z)$ and $\boldsymbol{T}^{\prime}(z)$ connected by Eq. (6.21) specify a given hypersurface relative to two choices of dust time, $\mathcal{T}$ and $\mathcal{T}^{\prime}$. Thus, the fiducial hypersurface $\boldsymbol{T}(z)=0$ of the time foliation $\mathcal{T}$ is also the hypersurface $\boldsymbol{T}^{\prime}(z)=\boldsymbol{\Theta}(z)$ of the time foliation $\mathcal{T}^{\prime}$.

The wave functional $\boldsymbol{\Psi}[Z, \boldsymbol{T}, \boldsymbol{g}]$ does not provide a unique description of the gravitational field on a given hypersurface for a second reason: The Lagrangian coordinates $z^{k}$ that label the dust worldlines are not unique. A relabeling of the dust worldlines is brought about by a diffeomorphism $\boldsymbol{\Xi}: \mathcal{S} \rightarrow \mathcal{S}$ of the dust space, as discussed in Secs. 2e and 5b. Under the action of $\boldsymbol{\Xi}$, the dust time $\boldsymbol{T}$ and the metric $\boldsymbol{g}$ change by the pullback mappings

$$
\boldsymbol{T}^{\prime}:=\boldsymbol{T} \circ \Xi, \quad \boldsymbol{g}^{\prime}:=\Xi^{*} \boldsymbol{g}
$$

According to Eq. (2.26), the action of a diffeomorphism $\boldsymbol{\Xi}$ on the dust frame $Z$ is given by $Z^{\prime}:=\Xi^{-1} \circ Z$.

The value of the state functional should not depend on the specification of the hypersurface. Thus, if $\boldsymbol{\Psi}[Z, \boldsymbol{T}, \boldsymbol{g}]$ describes the state of the gravitational field relative to the dust time $\mathcal{T}$ and $\boldsymbol{\Psi}^{\prime}\left[Z, \boldsymbol{T}^{\prime}, \boldsymbol{g}\right]$ describes the same state relative to the dust time $\mathcal{T}^{\prime}$, then these two wave functionals should be equal:

$$
\Psi^{\prime}\left[Z, \boldsymbol{T}^{\prime}=\boldsymbol{T}+\boldsymbol{\Theta}, \boldsymbol{g}\right]=\Psi[Z, \boldsymbol{T}, \boldsymbol{g}]
$$

Likewise, the value of the state functional should not depend on the choice of Lagrangian coordinate labels for the dust particles. Therefore,

$$
\Psi^{\prime}\left[Z^{\prime}=\boldsymbol{\Xi}^{-1} \circ Z, \boldsymbol{T}^{\prime}=\boldsymbol{T} \circ \boldsymbol{\Xi}, \boldsymbol{g}^{\prime}=\boldsymbol{\Xi}^{*} \boldsymbol{g}\right]=\boldsymbol{\Psi}[X, \boldsymbol{T}, \boldsymbol{g}]
$$

where $\boldsymbol{\Psi}[Z, \boldsymbol{T}, \boldsymbol{g}]$ describes the state of the gravitational field with respect to the Lagrangian coordinates $z^{k}$ and $\boldsymbol{\Psi}^{\prime}\left[Z^{\prime}, \boldsymbol{T}^{\prime}, \boldsymbol{g}^{\prime}\right]$ describes the same state with respect to the Lagrangian coordinates $z^{k^{\prime}}$.

These changes in the description of the state are generated by the Noether charges. Thus, the state $\boldsymbol{\Psi}^{\prime}$ from Eq. (6.23) can be obtained from the state $\boldsymbol{\Psi}$ by the action of the operator

$$
\hat{U}[\boldsymbol{\Theta}]:=\exp (-i \hat{Q}[\boldsymbol{\Theta}])
$$

generated by the Noether charge

$$
\hat{Q}[\boldsymbol{\Theta}]=\int_{\mathcal{S}} d^{3} z \boldsymbol{\Theta}(z) \hat{\boldsymbol{P}}(z)
$$

Indeed, we have

$$
\boldsymbol{\Psi}^{\prime}\left[Z, \boldsymbol{T}^{\prime}, \boldsymbol{g}\right]=\boldsymbol{\Psi}\left[Z, \boldsymbol{T}^{\prime}-\boldsymbol{\Theta}, \boldsymbol{g}\right]=\hat{U}[\boldsymbol{\Theta}] \boldsymbol{\Psi}\left[Z, \boldsymbol{T}^{\prime}, \boldsymbol{g}\right]
$$


Similarly, let $\boldsymbol{\Xi}^{k}(z, \sigma)$ denote a one-parameter group of diffeomorphisms with $\boldsymbol{\Xi}^{k}(z, 0)=z^{k}$ corresponding to the identify and $\boldsymbol{\Xi}^{k}(z, 1)=\boldsymbol{\Xi}^{k}(z)$ corresponding to a given final diffeomorphism. As usual, define the infinitesimal generators

$$
\boldsymbol{\xi}_{\sigma}^{k}(z):=\frac{d \Xi^{k}(z, \sigma)}{d \sigma}
$$

and the Noether charges $\hat{Q}\left[\overrightarrow{\boldsymbol{\xi}}_{\sigma}\right]$ corresponding to the classical charges (5.11). Then the state $\boldsymbol{\Psi}^{\prime}$ from Eq. (6.24) can be obtained from the state $\boldsymbol{\Psi}$,

$$
\boldsymbol{\Psi}^{\prime}\left[Z^{\prime}, \boldsymbol{T}^{\prime}, \boldsymbol{g}^{\prime}\right]=\boldsymbol{\Psi}\left[\boldsymbol{\Xi} \circ Z^{\prime}, \boldsymbol{T}^{\prime} \circ \boldsymbol{\Xi}^{-1},\left(\boldsymbol{\Xi}^{-1}\right)^{*} \boldsymbol{g}^{\prime}\right]=\hat{U}[\boldsymbol{\Xi}] \boldsymbol{\Psi}\left[Z^{\prime}, \boldsymbol{T}^{\prime}, \boldsymbol{g}^{\prime}\right]
$$

by the action of the operator

$$
\hat{U}[\boldsymbol{\Xi}]:=\mathbf{T}_{\sigma} \exp \left(-i \int_{0}^{1} d \sigma \hat{Q}\left[\overrightarrow{\boldsymbol{\xi}}_{\sigma}\right]\right) .
$$

Here, $\mathbf{T}_{\sigma}$ demands that the operators $\hat{Q}\left[\overrightarrow{\boldsymbol{\xi}}_{\sigma}\right]$ be ordered so that $\hat{Q}\left[\overrightarrow{\boldsymbol{\xi}}_{\sigma_{1}}\right]$ precedes $\hat{Q}\left[\overrightarrow{\boldsymbol{\xi}}_{\sigma_{2}}\right]$ if $\sigma_{2}>\sigma_{1}$.

The consistency of the formalism requires that physical states be transformed by the change of description again into physical states. Thus, if $\boldsymbol{\Psi}$ satisfies the constraints (6.1) and (6.3), the state $\boldsymbol{\Psi}^{\prime}$ defined by Eq. (6.23) should also satisfy the constraints. This follows from the commutator of the Noether charge (6.26) with the constraint operators:

$$
\left[\hat{Q}[\boldsymbol{\Theta}], \hat{\boldsymbol{H}}_{\uparrow}(z)\right]=0=\left[\hat{Q}[\Theta], \hat{H}_{\uparrow k}(x)\right] .
$$

Equations (6.27) and (6.31) imply

$$
\hat{\boldsymbol{H}}_{\uparrow}(z) \boldsymbol{\Psi}^{\prime}[Z, \boldsymbol{T}, \boldsymbol{g}]=\hat{U}[\boldsymbol{\Theta}] \hat{\boldsymbol{H}}_{\uparrow}(z) \boldsymbol{\Psi}[Z, \boldsymbol{T}, \boldsymbol{g}],
$$

and similarly for $\hat{H}_{\uparrow k}(x)$. Hence, if $\boldsymbol{\Psi}$ satisfies the constraints, $\boldsymbol{\Psi}^{\prime}$ also satisfies the constraints.

Similarly, if $\boldsymbol{\Psi}$ satisfies the constraints, then $\boldsymbol{\Psi}^{\prime}$ as defined in Eq. (6.24) should also satisfy them. This follows from the commutation relations

$$
\begin{aligned}
{\left[\hat{H}_{\uparrow k}(x), \hat{Q}[\overrightarrow{\boldsymbol{\xi}}]\right] } & =i \boldsymbol{\xi}_{, k}^{\ell}(\hat{Z}(x)) \hat{H}_{\uparrow \ell}(x), \\
{\left[\hat{\boldsymbol{H}}_{\uparrow}(z), \hat{Q}[\overrightarrow{\boldsymbol{\xi}}]\right] } & =i\left(\hat{\boldsymbol{H}}_{\uparrow}(z) \boldsymbol{\xi}^{k}(z)\right)_{, k},
\end{aligned}
$$

which are the quantum counterparts of the classical Poisson brackets $(5.13 \mathrm{~b}),(5.14)$ between $Q[\overrightarrow{\boldsymbol{\xi}}]$ and the constraints $H_{\uparrow k}(x), \boldsymbol{H}_{\uparrow}(z)$. Equations (6.33) show that if $\boldsymbol{\Psi}[Z, \boldsymbol{T}, \boldsymbol{g}]$ satisfies the constraints, then $\hat{Q}[\overrightarrow{\boldsymbol{\xi}}] \boldsymbol{\Psi}[Z, \boldsymbol{T}, \boldsymbol{g}]$ also satisfies the constraints. In turn, if $\hat{Q}[\overrightarrow{\boldsymbol{\xi}}] \boldsymbol{\Psi}[Z, \boldsymbol{T}, \boldsymbol{g}]$ satisfies the constraints, then $\boldsymbol{\Psi}^{\prime}[Z, \boldsymbol{T}, \boldsymbol{g}]=\hat{U}[\boldsymbol{\Xi}] \boldsymbol{\Psi}[Z, \boldsymbol{T}, \boldsymbol{g}]$ also satisfies the constraints. We conclude that the description of a physical state can be changed by the action of the operators $\hat{U}[\boldsymbol{\Theta}]$ and $\hat{U}[\boldsymbol{\Xi}]$ generated by the Noether charges. 


\section{E. The Inner Product}

The transition from the Hamiltonian constraint $H_{\perp}(x)$ to the new constraint $\boldsymbol{H}_{\uparrow}(z)$ leads, upon quantization, to the replacement of the Wheeler-DeWitt equation by the functional Schrödinger equation (6.5). Unlike the Wheeler-DeWitt equation, the Schrödinger equation has (at least formally) a conserved positive-definite inner product [3]. This, of course, is the main motivation behind rewriting the Hamiltonian constraint in the forms (4.6) and (5.8).

Let $\boldsymbol{\Psi}[\boldsymbol{g}]$ be a functional of Riemannian metrics $\boldsymbol{g}(z) \in \operatorname{Riem} \mathcal{S}$ in the dust space $\mathcal{S}$. The inner product between two such functionals is defined by the functional integral

$$
\left\langle\Psi_{1} \mid \Psi_{2}\right\rangle:=\int_{\text {Riem } \mathcal{S}} \operatorname{Dg} \bar{\Psi}_{1}[\boldsymbol{g}] \Psi_{2}[g]
$$

Those functionals $\boldsymbol{\Psi}[\boldsymbol{g}]$ that are square integrable under the product (6.34) form a Hilbert space $\mathcal{H}$.

The functionals $\boldsymbol{\Psi}[\boldsymbol{g}]$ can be interpreted as states of the gravitational field on a fixed initial hypersurface $\boldsymbol{T}_{0}(z)$ :

$$
\Psi\left[\boldsymbol{T}_{0}, \boldsymbol{g}\right]:=\Psi[g]
$$

By virtue of their independence from $Z$, the state functionals $\boldsymbol{\Psi}\left[\boldsymbol{T}_{0}, \boldsymbol{g}\right]$ automatically satisfy the operator constraints (6.3). Let us evolve these states by the Schrödinger equation (6.5). The solutions $\boldsymbol{\Psi}[\boldsymbol{T}, \boldsymbol{g}]$, from Eq. (6.19), form a space which we call $\mathcal{H}_{\uparrow}$. By fixing $\boldsymbol{T}(z)$ to be the given initial hypersurface $\boldsymbol{T}_{0}(z)$, a functional $\boldsymbol{\Psi}[\boldsymbol{T}, \boldsymbol{g}]$ from $\mathcal{H}_{\uparrow}$ is mapped to a functional (6.35) from $\mathcal{H}$.

We now ask whether the inner product

$$
\left\langle\Psi_{1} \mid \Psi_{2}\right\rangle:=\int_{\text {RiemS }} \operatorname{Dg} \bar{\Psi}_{1}[\boldsymbol{T}, \boldsymbol{g}] \boldsymbol{\Psi}_{2}[\boldsymbol{T}, \boldsymbol{g}]
$$

between two states from the solution space $\mathcal{H}_{\uparrow}$ depends on the choice of hypersurface $\boldsymbol{T}(z)$. Formally, the answer is no, provided that the Hamiltonian density $\boldsymbol{h}(z ; \hat{\boldsymbol{g}}, \hat{\boldsymbol{p}}]$ of Eq. (6.5) is a self-adjoint operator under the inner product (6.34). However, $\hat{\boldsymbol{h}}$ is not self-adjoint on the whole space $\mathcal{H}$, but only on the linear subspace $\mathcal{H}^{+} \subset \mathcal{H}$ which is spanned by the simultaneous eigenfunctions (6.13) of the operators $\hat{\boldsymbol{G}}(z)$ with positive eigenvalues $\boldsymbol{G}(z)>0$. Hence, if the inner product (6.36) is to be independent of the choice of hypersurface, we must limit the solution space $\mathcal{H}_{\uparrow}$ to $\mathcal{H}_{\uparrow}^{+}$.

To summarize, the state of the gravitational field can be represented either as an element of the space of solutions $\mathcal{H}_{\uparrow}^{+}$or as an element of the space of initial states $\mathcal{H}^{+}$. Conservation of the inner product guarantees that the value obtained for the inner product is the same irrespective of the representation.

\section{F. Observables}

Any functional $\boldsymbol{F}[\boldsymbol{g}, \boldsymbol{p}]$ of the metric $\boldsymbol{g}(z)$ and extrinsic curvature $\boldsymbol{p}(z)$ represents a gravitational dynamical variable that is invariant under Diff $\Sigma$. We will call the space of such variables $\mathcal{F}$. If the dust particles are labeled by a fixed set of Lagrangian coordinates $z^{k}$, 
then in principle the full metric $\boldsymbol{g}_{k \ell}(z)$ on a given hypersurface, not just the intrinsic geometry of the hypersurface, can be measured. A corresponding statement holds for the extrinsic curvature. If we want, we can restrict our attention to variables in $\mathcal{F}$ that do not depend on the choice of Lagrangian coordinates for the dust; such variables have vanishing Poisson brackets with the generator $Q[\overrightarrow{\boldsymbol{\xi}}]$ of dust space diffeomorphisms. In the following, it makes no difference whether we take the position that all the dynamical variables in $\mathcal{F}$ are measurable, or only those that are invariant under Diff $\mathcal{S}$. Thus, we leave open the issue of which class of variables should be called observables.

In quantum theory, an observable from $\mathcal{F}$ is represented by an operator

$$
\hat{\boldsymbol{F}}=\boldsymbol{F}[\hat{\boldsymbol{g}}, \hat{\boldsymbol{p}}]
$$

that acts on $\mathcal{H}$ and is self-adjoint under the inner product (6.34). The statistical interpretation of quantum geometrodynamics is based on two fundamental assumptions. The first one is the mean value formula: When $\boldsymbol{F}$ is measured on an ensemble of systems described by the state functional $\boldsymbol{\Psi}\left[\boldsymbol{T}_{0}, \boldsymbol{g}\right] \in \mathcal{H}$ on a hypersurface $\boldsymbol{T}_{0}(z)$, the mean value of the result is given by the expression

$$
\langle\boldsymbol{F}\rangle=\langle\boldsymbol{\Psi} \mid \hat{\boldsymbol{F}} \boldsymbol{\Psi}\rangle
$$

with the inner product (6.34). By the standard argument, it follows that the permissible values of $\boldsymbol{F}$ are the eigenvalues of $\hat{\boldsymbol{F}}$ and, if the system is in one of the eigenstates of $\hat{\boldsymbol{F}}$, the measurement of $\hat{\boldsymbol{F}}$ on the hypersurface $\boldsymbol{T}_{0}(z)$ yields the corresponding eigenvalue with certainty. The second assumption is the reduction postulate: If $\hat{\boldsymbol{F}}$ is measured on a hypersurface $\boldsymbol{T}_{0}(z)$ and those systems for which the measurement yields the eigenvalue $\boldsymbol{F}^{\prime}$ are collected into a new ensemble, then the state functional $\boldsymbol{\Psi}^{\prime}\left[\boldsymbol{T}_{0}, \boldsymbol{g}\right]$ that describes the new ensemble is the orthogonal projection (under the inner product (6.34)) of the original state $\boldsymbol{\Psi}\left[\boldsymbol{T}_{0}, \boldsymbol{g}\right]$ to the linear subspace of $\mathcal{H}$ spanned by the eigenstates of $\hat{\boldsymbol{F}}$ belonging to the eigenvalue $\boldsymbol{F}^{\prime}$.

So far, observables have been represented by operators acting on a state $\boldsymbol{\Psi}\left[\boldsymbol{T}_{0}, \boldsymbol{g}\right] \in \mathcal{H}$ at the time of measurement $\boldsymbol{T}_{0}(z)$. The functional

$$
\boldsymbol{\Phi}\left[\boldsymbol{T}_{0}, \boldsymbol{g}\right]=\hat{\boldsymbol{F}} \boldsymbol{\Psi}\left[\boldsymbol{T}_{0}, \boldsymbol{g}\right]
$$

lies in the same instantaneous Hilbert space $\mathcal{H}$ as the original functional $\Psi$. Let us consider the functionals $\boldsymbol{\Psi}$ and $\boldsymbol{\Phi}$ as initial data and evolve them by the Schrödinger equation (6.5) away from the initial hypersurface. In this way, we obtain states (6.19) that belong to the solution space $\mathcal{H}_{\uparrow}$ :

$$
\begin{aligned}
& \Psi[\boldsymbol{T}, \boldsymbol{g}]=\boldsymbol{U}\left[\boldsymbol{T}-\boldsymbol{T}_{0} ; \hat{\boldsymbol{g}}, \hat{\boldsymbol{p}}\right] \boldsymbol{\Psi}\left[\boldsymbol{T}_{0}, \boldsymbol{g}\right] \\
& \boldsymbol{\Phi}[\boldsymbol{T}, \boldsymbol{g}]=\boldsymbol{U}\left[\boldsymbol{T}-\boldsymbol{T}_{0} ; \hat{\boldsymbol{g}}, \hat{\boldsymbol{p}}\right] \boldsymbol{\Phi}\left[\boldsymbol{T}_{0}, \boldsymbol{g}\right]
\end{aligned}
$$

The state (6.41) is obtained from the state (6.40) by the action of the operator

$$
\boldsymbol{F}_{\mathbf{T}_{0}}[\boldsymbol{T} ; \hat{\boldsymbol{g}}, \hat{\boldsymbol{p}}]=\boldsymbol{U}\left[\boldsymbol{T}-\boldsymbol{T}_{0} ; \hat{\boldsymbol{g}}, \hat{\boldsymbol{p}}\right] \boldsymbol{F}[\hat{\boldsymbol{g}}, \hat{\boldsymbol{p}}] \boldsymbol{U}^{-1}\left[\boldsymbol{T}-\boldsymbol{T}_{0} ; \hat{\boldsymbol{g}}, \hat{\boldsymbol{p}}\right] .
$$

Equation (6.42) represents the observable $\boldsymbol{F}[\boldsymbol{g}, \boldsymbol{p}]$ which is measured on the hypersurface $\boldsymbol{T}_{0}(z)$ as an operator $\hat{\boldsymbol{F}}_{\mathbf{T}_{0}}$ on the solution space $\mathcal{H}_{\uparrow}$. The operator $\hat{\boldsymbol{F}}_{\mathbf{T}_{0}}$ is constructed so that it commutes with the Hamiltonian constraint $\hat{\boldsymbol{H}}_{\uparrow}(z)$ : 


$$
\left[\hat{\boldsymbol{F}}_{\mathbf{T}_{0}}, \hat{\boldsymbol{H}}_{\uparrow}(z)\right]=0
$$

This property guarantees that the action of $\hat{\boldsymbol{F}}_{\mathbf{T}_{0}}$ on a state in $\mathcal{H}_{\uparrow}$ again yields a state in $\mathcal{H}_{\uparrow}$.

We must now translate into the quantum theory the positivity condition $\boldsymbol{G}(z ; \boldsymbol{g}, \boldsymbol{p}]>0$ on the domain of classical observables $\boldsymbol{F}[\boldsymbol{g}, \boldsymbol{p}]$. Quantum mechanically, the action of an operator $\boldsymbol{F}[\hat{\boldsymbol{g}}, \hat{\boldsymbol{p}}]$ on a state $\boldsymbol{\Psi}[\boldsymbol{g}] \in \mathcal{H}^{+}$from the subspace $\mathcal{H}^{+}$spanned by the eigenfunctionals of the operators $\boldsymbol{G}(z ; \hat{\boldsymbol{g}}, \hat{\boldsymbol{p}}]$ with positive eigenvalues $\boldsymbol{G}(z)>0$ should leave the state in $\mathcal{H}^{+}$. If an observable $\hat{\boldsymbol{F}} \in \hat{\mathcal{F}}$ does not have this property, the reduction postulate leads us into difficulty. The reduced state $\boldsymbol{\Psi}^{\prime}\left[\boldsymbol{T}_{0}, \boldsymbol{g}\right]$ obtained after the measurement of such an observable would not lie in $\mathcal{H}^{+}$. Therefore, if we measure $\hat{\boldsymbol{G}}(z)$ on the reduced state $\boldsymbol{\Psi}^{\prime}$, there would be a nonvanishing probability of finding $\hat{\boldsymbol{G}}(z)$ in the unphysical region $\boldsymbol{G}(z) \leq 0$. Furthermore, by allowing the reduced state $\boldsymbol{\Psi}^{\prime}\left[\boldsymbol{T}_{0}, \boldsymbol{g}\right]$ to propagate to a later time $\boldsymbol{T}(z)>\boldsymbol{T}_{0}(z)$ via the evolution operator $\boldsymbol{U}\left[\boldsymbol{T}-\boldsymbol{T}_{0} ; \hat{\boldsymbol{g}}, \hat{\boldsymbol{p}}\right]$, we would find that the norm $\left\langle\boldsymbol{\Psi}^{\prime}[\boldsymbol{T}, \boldsymbol{g}] \mid \boldsymbol{\Psi}^{\prime}[\boldsymbol{T}, \boldsymbol{g}]\right\rangle$ depends on $\boldsymbol{T}(z)$ and the total probability is not conserved.

These considerations force us to define observables as self-adjoint operators $\hat{\boldsymbol{F}} \in \hat{\mathcal{F}}^{+}$on $\mathcal{H}^{+}$rather than on $\mathcal{H}$. That is, an operator $\hat{\boldsymbol{F}}$ is considered to be an observable only if its action on the states $\boldsymbol{\Psi}$ from $\mathcal{H}^{+}$again yield states in $\mathcal{H}^{+}$. Correspondingly, the observables $\hat{\boldsymbol{F}}_{\mathbf{T}_{0}}$ are self-adjoint operators on the space of solutions $\mathcal{H}_{\uparrow}^{+}$. Thus, for $\boldsymbol{\Psi}$ an element of $\mathcal{H}_{\uparrow}^{+}$, the state $\hat{\boldsymbol{F}}_{\mathbf{T}_{0}} \boldsymbol{\Psi}$ also is an element of $\mathcal{H}_{\uparrow}^{+}$. This implies that the mean value of $\hat{\boldsymbol{F}}_{\mathbf{T}_{0}}$, defined by

$$
\left\langle\boldsymbol{F}_{\mathbf{T}_{0}}\right\rangle=\left\langle\Psi \mid \hat{\boldsymbol{F}}_{\mathbf{T}_{0}} \boldsymbol{\Psi}\right\rangle
$$

can be evaluated on any hypersurface, not only at the instant $\boldsymbol{T}_{0}(z)$ of the measurement.

The condition for an operator defined on $\mathcal{H}$ to be an observable can be expressed in terms of the projection operator

$$
\hat{\mathcal{P}}^{+}:=\prod_{z \in \mathcal{S}} \Theta(\hat{\boldsymbol{G}}(z))
$$

from $\mathcal{H}$ to $\mathcal{H}^{+}$. The projector $\hat{\mathcal{P}}^{+}$is a product of step functions $\Theta$ of the commuting operators $\hat{\boldsymbol{G}}(z)$. The Hilbert space $\mathcal{H}^{+}$is spanned by states of the form $\hat{\mathcal{P}}^{+} \boldsymbol{\Psi}$, where $\boldsymbol{\Psi} \in \mathcal{H}$. An operator $\hat{\boldsymbol{F}}$ on $\mathcal{H}$ is an observable if it satisfies the condition

$$
\left[\hat{\boldsymbol{F}}, \hat{\mathcal{P}}^{+}\right] \hat{\mathcal{P}}^{+}=\left(1-\hat{\mathcal{P}}^{+}\right) \hat{\boldsymbol{F}} \hat{\mathcal{P}}^{+}=0
$$

Thus, any variable $\boldsymbol{F}[\hat{\boldsymbol{g}}, \hat{\boldsymbol{p}}]$ that commutes with $\hat{\boldsymbol{G}}(z)$, i.e., that is a constant of motion, is automatically an observable. However, $\hat{\boldsymbol{F}}$ does not need to be a constant of motion to satisfy Eq. (6.46); observables do not need to be constants of motion.

The fundamental problem in interpreting canonical quantum gravity through its coupling to dust is the construction of meaningful gravitational observables. Ultimately, one would like to exhibit complete sets of commuting observables.

On our way, we met some gravitational observables. The operators $G(z ; \hat{\mathbf{g}}, \hat{\mathbf{p}}]$, equivalent to the energy densities $h(z ; \mathbf{g}, \mathbf{p}]$, form a commuting set of gravitational observables on $\mathcal{H}^{+}$. The Noether charges $\hat{Q}[\overrightarrow{\boldsymbol{\xi}}]$ and $\hat{Q}[\boldsymbol{\Theta}]$ represent observables on $\mathcal{H}_{\uparrow}^{+}$. By using the constraints, we can eliminate the embedding momenta and express the charges in terms of 
the gravitational variables. There are thus some observables we can measure and subject to statistical analysis. All of these are constants of motion.

It is disappointing that the fundamental gravitational variables $\hat{\boldsymbol{g}}(z)=\boldsymbol{g}(z) \times$ and $\hat{\boldsymbol{p}}(z)=$ $-i \delta / \delta \boldsymbol{g}(z)$ are not gravitational observables. Although the condition (6.46) is cumbersome to handle explicitly, it is nevertheless fairly obvious that $\hat{\boldsymbol{g}}(z)$ and $\hat{\boldsymbol{p}}(z)$ do not necessarily leave the states in $\mathcal{H}^{+}$. The underlying difficulty is that the gravitational Hamiltonian $h(x ; \hat{\boldsymbol{g}}, \hat{\boldsymbol{p}}]$ is not real by its construction on the entire Hilbert space $\mathcal{H}$ which carries the representation of the fundamental operators $\hat{\boldsymbol{g}}(z)$ and $\hat{\boldsymbol{p}}(z)$.

One can illustrate these problems on simple finite-dimensional models, like a relativistic particle in the proper-time formalism (Appendix G). In particular, one can explicitly verify that even on a flat Minkowskian background the multiplication position operator is not an observable. One may hope to clarify the status of fundamental variables on such simple models. For the time being, their relation to quantum observables remains unclear.

\section{G. Quantum Theory in Terms of State Functionals $\Psi[Z, T, g]$}

The quantum theory of gravity coupled to dust, as developed thus far, can be expressed in terms of the original set of canonical variables $Z^{k}(x), P_{k}(x), T(x), P(x), g_{a b}(x)$, and $p^{a b}(x)$. In this case, the quantum states are functionals $\Psi[Z, T, g]$ that satisfy the operator constraint equations

$$
\begin{aligned}
H_{\uparrow}\left(x ; \hat{P}, \hat{g}_{a b}, \hat{p}^{a b}\right] \Psi[Z, T, g] & =0, \\
H_{\uparrow k}\left(x ; \hat{Z}^{k}, \hat{P}_{k}, \hat{T}, \hat{g}_{a b}, \hat{p}^{a b}\right] \Psi[Z, T, g] & =0 .
\end{aligned}
$$

Let us assume that $\boldsymbol{\Psi}[\boldsymbol{T}, \boldsymbol{g}]$ is a solution to the functional Schrödinger equation (6.1). Recall that such a functional automatically satisfies the operator constraint (6.3) by virtue of its lack of dependence on $Z^{k}(x)$. Now choose a mapping $Z: \Sigma \rightarrow \mathcal{S}$ that associates the points in space with particles of dust, and consider the functional defined by

$$
\Psi[Z, T, g]:=\Psi\left[\left(Z^{-1}\right)^{*} T,\left(Z^{-1}\right)^{*} g\right] .
$$

Here, $\boldsymbol{T}=\left(Z^{-1}\right)^{*} T$ and $\boldsymbol{g}=\left(Z^{-1}\right)^{*} g$ denote the pullbacks by $X=Z^{-1}$ of the space tensors $T$ and $g$ to the dust space $\mathcal{S}$. As we show below, the functional (6.48) satisfies the constraints (6.47) and represents the quantum state of the system in terms of the original canonical coordinates.

Consider first the supermomentum operator constraint (6.47b). Since $\hat{H}_{\uparrow k}(x)=$ $\hat{Z}_{k}^{a}(x) \hat{H}_{a}(x)$, this equation implies $\hat{H}_{a}(x) \Psi=0$ which in turn implies that $\Psi$ is invariant under spatial diffeomorphisms. Indeed, let $\varphi_{\sigma}: \Sigma \rightarrow \Sigma$ denote a one-parameter family of diffeomorphisms generated by the vector field $\vec{N}(x)$. Assuming an appropriate factor ordering, we have

$$
\left.\frac{d}{d \sigma} \Psi\left[\varphi_{\sigma}^{*} Z, \varphi_{\sigma}^{*} T, \varphi_{\sigma}^{*} g\right]\right|_{\sigma=0}=i \int_{\Sigma} d^{3} x N^{a}(x) \hat{H}_{a}(x) \Psi[Z, T, g]=0
$$

for any wave functional that satisfies the supermomentum operator constraint. By exponentiation in $\sigma$, this result yields 


$$
\Psi\left[\varphi^{*} Z, \varphi^{*} T, \varphi^{*} g\right]=\Psi[Z, T, g]
$$

and shows that $\Psi$ is invariant under finite diffeomorphisms of $\Sigma$. Conversely, a diffeomorphism invariant functional (6.50) will satisfy the supermomentum constraint (6.47b). The functional $\Psi$ defined in Eq. (6.48) is just such an invariant functional. This follows from the fact that $\left(Z^{-1}\right)^{*} T$ and $\left(Z^{-1}\right)^{*} g$ are invariant under the action of Diff $\Sigma$ : For $\left(Z^{-1}\right)^{*} T$, we have

$$
\begin{aligned}
\left(\left(\varphi^{*} Z\right)^{-1}\right)^{*}\left(\varphi^{*} T\right) & =(T \circ \varphi) \circ(Z \circ \varphi)^{-1}=T \circ Z^{-1} \\
& =\left(Z^{-1}\right)^{*} T
\end{aligned}
$$

and similarly for $\left(Z^{-1}\right)^{*} g$. Therefore the state functional $\Psi[Z, T, g]$ defined in Eq. (6.48) satisfies the supermomentum constraint (6.47b).

The functional (6.48), which we assume satisfies the functional Schrödinger equation (6.1), also satisfies the functional Schrödinger equation (6.47a). In order to understand this result, it is only necessary to clarify the relationship between the operators $\hat{P}, \hat{g}$, and $\hat{p}$ and their dust space counterparts $\hat{\boldsymbol{P}}, \hat{\boldsymbol{g}}$, and $\hat{\boldsymbol{p}}$. We will assume that these sets of operators are related by the familiar mapping of tensor fields by $Z$. For example, the action of $\hat{P}(x)$ and the action of $\hat{\boldsymbol{P}}(z)$ are related by

$$
\hat{P}(x) \Psi\left[\left(Z^{-1}\right)^{*} T,\left(Z^{-1}\right)^{*} g\right]=\left.\left|\frac{\partial Z(x)}{\partial x}\right|(\hat{\boldsymbol{P}}(z) \boldsymbol{\Psi}[\boldsymbol{T}, \boldsymbol{g}])\right|_{z=Z(x), \mathbf{T}=\left(Z^{-1}\right)^{*} T, \mathbf{g}=\left(Z^{-1}\right)^{*} g} .
$$

There are no factor ordering ambiguities in this relationship. Using an abbreviated notation, we can write $\hat{P}(x)=|\partial Z(x) / \partial x| \hat{\boldsymbol{P}}(Z(x))$. Observe that if the operator $\hat{P}(x)$ is represented by $-i \delta / \delta T(x)$ then $\hat{\boldsymbol{P}}(z)$ is represented by $-i \delta / \delta \boldsymbol{T}(z)$. A relationship analogous to that expressed in Eq. (6.52) can be used to define a correspondence between functions of the operators $\hat{\boldsymbol{P}}, \hat{\boldsymbol{g}}$, and $\hat{\boldsymbol{p}}$, and functions of the operators $\hat{P}, \hat{g}$, and $\hat{p}$. In particular, the new super-Hamiltonian operator is (using the abbreviated notation)

$$
H_{\uparrow}(x ; \hat{P}, \hat{g}, \hat{p}]=\left|\frac{\partial Z(x)}{\partial x}\right| \boldsymbol{H}_{\uparrow}(Z(x) ; \hat{\boldsymbol{P}}, \hat{\boldsymbol{g}}, \hat{\boldsymbol{p}}] .
$$

From this relationship we obtain the correspondence

$$
\hat{H}_{\uparrow}(x) \boldsymbol{\Psi}\left[\left(Z^{-1}\right)^{*} T,\left(Z^{-1}\right)^{*} g\right]=0 \quad \Longleftrightarrow \quad \hat{\boldsymbol{H}}_{\uparrow}(z) \boldsymbol{\Psi}[\boldsymbol{T}, \boldsymbol{g}]=0 .
$$

Therefore, if $\boldsymbol{\Psi}[\boldsymbol{T}, \boldsymbol{g}]$ satisfies the Schrödinger equation (6.1), then $\Psi[Z, T, g]$ as defined in Eq. (6.48) satisfies the Schrödinger equation (6.47a).

The preceding arguments show that the state functional $\boldsymbol{\Psi}[\boldsymbol{T}, \boldsymbol{g}]$ can be expressed as a functional $\Psi$ of $T, g$, and a mapping $Z: \Sigma \rightarrow \mathcal{S}$ through definition (6.48). Different choices of mappings $Z$ lead to different functionals $\Psi$; however, these functionals are related by the unitary transformation $\hat{U}[\boldsymbol{\Xi}]$ from Eq. (6.30) and represent the same physical quantum state.

The spectral analysis, the construction of the Hilbert space and inner product, and the definition of observables can be translated into the language of the original canonical variables in a straightforward manner. In particular, the Hilbert space $\mathcal{H}^{+}$is spanned by 
the eigenfunctionals $\phi\left[\left(Z^{-1}\right)^{*} G ;\left(Z^{-1}\right)^{*} g\right]$ of $\hat{G}(x)$ with positive eigenvalues $G(x)>0$. The inner product between two states of the form (6.48) is

$$
\begin{aligned}
\left\langle\Psi_{1} \mid \Psi_{2}\right\rangle & :=\int_{\text {RiemS }} \mathrm{D} \boldsymbol{g} \overline{\boldsymbol{\Psi}}_{1}\left[\left(Z^{-1}\right)^{*} T, \boldsymbol{g}\right] \boldsymbol{\Psi}_{2}\left[\left(Z^{-1}\right)^{*} T, \boldsymbol{g}\right] \\
& =\int_{\operatorname{Riem} \Sigma} \mathrm{D} g \bar{\Psi}_{1}[Z, T, g] \Psi_{2}[Z, T, g]
\end{aligned}
$$

Finally, the gravitational observables are operators $F[\hat{Z}, \hat{g}, \hat{p}]$ that correspond, in the sense of Eq. (6.52), to operators $\boldsymbol{F}[\hat{\boldsymbol{g}}, \hat{\boldsymbol{p}}]$. Their action on a state vector in the Hilbert space $\mathcal{H}^{+}$ again yields a state in $\mathcal{H}^{+}$.

\section{NON-ROTATING DUST}

\section{A. Rotation and Vorticity}

In general, no dust time $\mathcal{T}$ is better than any other. However, if the dust does not rotate as it moves, so that the four-velocity field is hypersurface orthogonal, then there is a privileged time foliation $\mathcal{T}_{\perp}$, namely, the foliation by hypersurfaces orthogonal to the flow lines. For such a foliation, $W_{k}=0$.

The rotation of the dust is measured by the vorticity 2 -form

$$
\Omega_{\alpha \beta}:=\left(\delta_{\alpha}^{\gamma}+U^{\gamma} U_{\alpha}\right)\left(\delta_{\beta}^{\delta}+U^{\delta} U_{\beta}\right) U_{[\gamma, \delta]} .
$$

For $\Omega_{\alpha \beta}=0$, the vector field $U^{\alpha}$ is hypersurface orthogonal. The projectors in Eq. (7.1) ensure that $\Omega_{\alpha \beta} U^{\beta} \equiv 0$; hence, $\Omega_{\alpha \beta}$ is completely characterized by its orthogonal projection $\Omega_{k \ell}:=\Omega_{\alpha \beta} \Upsilon_{, k}^{\alpha} \Upsilon_{, \ell}^{\beta}$. The equations of motion (2.17)-(2.18) imply that

$$
\boldsymbol{\Omega}_{k \ell}=\boldsymbol{W}_{[k, \ell]} .
$$

Like $\boldsymbol{W}_{k}$ itself, $\boldsymbol{\Omega}_{k \ell}$ is conserved along the flow lines of $U^{\alpha}$ :

$$
£_{U} \Omega_{k \ell}=\Omega_{k \ell, \alpha} U^{\alpha}=0 .
$$

Hence, if the dust is irrotational at a spacetime point $y$, then the equations of motion ensure that it is irrotational for all points along the flow line that passes through $y$.

The projected vorticity $(7.2)$ is an exact two-form in the dust space $\mathcal{S}$. Moreover, it is invariant under the symmetry transformation (2.27). If $\boldsymbol{\Omega}_{k \ell}$ vanishes, the velocity field $\boldsymbol{W}_{k}$ is closed; thus $\boldsymbol{W}_{k}$ can be (at least locally) transformed to zero by the symmetry transformation (2.27). This transformation brings us from a foliation $\mathcal{T}$ to the privileged time foliation $\mathcal{T}_{\perp}$.

The foliation $\mathcal{T}_{\perp}$ also can be constructed by choosing an initial hypersurface orthogonal to the flow lines and then Lie propagating it along $U^{\alpha}$. Because $U^{\alpha}$ is a geodesic vector field, Eq. (2.25), the propagated hypersurface stays orthogonal to $U^{\alpha}$. This amounts to the familiar construction of a Gaussian coordinate system [22]. The coordinate system $\left(\tau, z^{k}\right)$ based on the time $\mathcal{T}_{\perp}$ and space $\mathcal{S}$ manifolds of an irrotational dust is a Gaussian coordinate system. 


\section{B. Canonical Description of Non-Rotating Dust}

The pullback of the vorticity 2 -form (7.2) from $\mathcal{S}$ to $\Sigma$ is

$$
\begin{aligned}
\Omega_{a b} & :=\Omega_{k \ell} Z_{, a}^{k} Z_{, b}^{\ell}=\left(W_{k} Z_{,[a}^{k}\right)_{, b]} \\
& =U_{[a, b]} .
\end{aligned}
$$

Note that in general the direct projection of the spacetime vorticity tensor (7.1) onto $\Sigma$ differs from the spatial tensor $\Omega_{a b}$ by a term $A_{[a} U_{b]}$, where $A_{a}$ is the spatial projection of the dust acceleration $A_{\alpha}=U^{\beta} \nabla_{\beta} U_{\alpha}$. When the Euler equations of motion hold, the acceleration (2.25) vanishes and the spatial projection of the spacetime vorticity coincides with the vorticity $\Omega_{a b}$ of Eq. (7.4).

Under spatial diffeomorphisms, $\Omega_{a b}(x)$ transforms as a tensor; this determines its Poisson bracket with the supermomentum $H_{a}^{D}(x)$. The Poisson bracket of $\Omega_{a b}(x)$ and $\Omega_{c d}\left(x^{\prime}\right)$ vanishes:

$$
\left\{\Omega_{a b}(x), \Omega_{c d}\left(x^{\prime}\right)\right\}=0 .
$$

Finally, the Poisson bracket of $\Omega_{a b}(x)$ and the super-Hamiltonian is

$$
\left\{\Omega_{a b}(x), H_{\perp}^{D}\left(x^{\prime}\right)\right\}=-2 \frac{H^{D c}\left(x^{\prime}\right)}{H_{\perp}^{D}\left(x^{\prime}\right)} \Omega_{c[a}\left(x^{\prime}\right) \delta_{, b]}\left(x, x^{\prime}\right) .
$$

This result is obtained as follows. Because neither $Z^{k}(x)$ nor $W_{k}(x)=-P_{k}(x) / P(x)$ depend on $T(x)$, they have vanishing Poisson brackets with $P\left(x^{\prime}\right)$. The only part of the superHamiltonian $H_{\perp}^{D}\left(x^{\prime}\right)$ that affects these variables is the dust momentum $H_{a}^{D}\left(x^{\prime}\right)$ in the squareroot energy-density (3.19). The momentum $H_{a}^{D}\left(x^{\prime}\right)$ generates the transformation of $Z^{k}(x)$ and $W_{k}(x)$ as scalars under Diff $\Sigma$. Therefore,

$$
\begin{aligned}
& \left\{W_{k}(x), H_{\perp}^{D}\left(x^{\prime}\right)\right\}=W_{k, b}(x) S^{b}(x) \delta\left(x, x^{\prime}\right), \\
& \left\{Z^{k}(x), H_{\perp}^{D}\left(x^{\prime}\right)\right\}=Z^{k}{ }_{, b}(x) S^{b}(x) \delta\left(x, x^{\prime}\right),
\end{aligned}
$$

with

$$
S^{b}(x):=\frac{H^{D b}(x)}{H_{\perp}^{D}(x)} .
$$

Equations (7.7) allow us to calculate the Poisson bracket

$$
\begin{aligned}
\left\{W_{k}(x) Z_{, a}^{k}(x), H_{\perp}^{D}\left(x^{\prime}\right)\right\} & =2 \Omega_{a b}\left(x^{\prime}\right) S^{b}\left(x^{\prime}\right) \delta\left(x, x^{\prime}\right) \\
+ & \left(U_{k}(x) Z^{k}{ }_{, b}(x) S^{b}(x) \delta\left(x, x^{\prime}\right)\right)_{, a}
\end{aligned}
$$

where $\Omega_{a b}$ is given by Eq. (7.4). By taking the exterior derivative of Eq. (7.9), we obtain Eq. (7.6) above.

The results (7.5)-(7.6) show that if a new constraint

$$
\Omega_{a b}(x)=0
$$


is adjoined to the super-Hamiltonian and supermomentum constraints (3.20) and (3.21), then the enlarged system of constraints is first class. Moreover, from Eq. (3.14) we have

$$
U_{a}=-\frac{H_{a}^{D}}{P}
$$

It is easy to see that both $P$ and $H_{a}^{D}$ are invariant under the symmetry transformations, i.e., that their Poisson brackets with the generators (3.24) and (3.25) vanish. Therefore $U_{a}$ and $\Omega_{a b}$ are also invariant under the symmetries:

$$
\left\{\Omega_{a b}(x), Q[\boldsymbol{\vartheta}]\right\}=0=\left\{\Omega_{a b}(x), Q[\overrightarrow{\boldsymbol{\xi}}]\right\} .
$$

Thus, the new constraint (7.10) is invariant under the symmetry transformations (3.24), (3.25). The condition (7.10) constrains the dust into irrotational flow. The first-class nature of the enlarged system of constraints ensures that if the dust is irrotational on an initial hypersurface, its dynamics prevents the evolution of vorticity.

Let us study the vorticity potential $W_{k}(x):=-P_{k}(x) / P(x)$ from the canonical point of view. The Poisson bracket of $W_{k}(x)$ with the super-Hamiltonian $H_{\perp}(x)$ is different from zero; on the other hand, its Poisson bracket with the new constraint $H_{\uparrow}(x)$ vanishes:

$$
\left\{W_{k}(x), H_{\uparrow}\left(x^{\prime}\right)\right\}=0 .
$$

This reflects the fact that $W_{k}(x)$ does not change along the worldlines of the dust although it does change along the normal direction to an embedding. We can now solve the Heisenberg equations of motion (4.18) for the dynamical variables $W_{k}(x)$. Any such solution is a functional $W_{k}\left(x ; Z^{L}\right]$ of the embedding $Z^{L}=\left(T(x), Z^{\ell}(x)\right)$, as expressed in Eq. (4.16). When we substitute Eq. (7.13) into the $T$-component of the Heisenberg equations of motion, we learn that $W_{k}\left(Z^{L}(x)\right)$ cannot depend on $T(x)$, i.e., that $W_{k}=W_{k}\left(x ; Z^{\ell}\right]$. Since $W_{k}(x):=$ $-P_{k}(x) / P(x)$ is a spatial scalar, it follows that

$$
\left\{W_{k}(x), H_{a}\left(x^{\prime}\right)\right\}=W_{k, a}(x) \delta\left(x, x^{\prime}\right) .
$$

The definition (4.9) of $H_{\uparrow k}(x)$ then implies that weakly, modulo the supermomentum constraint,

$$
\left\{W_{k}(x), H_{\uparrow \ell}\left(x^{\prime}\right)\right\}=W_{k, a}(x) Z_{\ell}^{a}(x) \delta\left(x, x^{\prime}\right) .
$$

The $Z^{\ell}$-component of the Heisenberg equations of motion (4.18) thus reads

$$
\frac{d W_{k}\left(x ; Z^{m}\right]}{\delta Z^{\ell}\left(x^{\prime}\right)}=W_{k, a}(x) Z_{\ell}^{a}(x) \delta\left(x, x^{\prime}\right) .
$$

The delta function on the right-hand side of Eq. (7.16) tells us that $W_{k}\left(x ; Z^{\ell}\right]$ is an ultralocal functional of $Z^{\ell}(x)$, i.e., that

$$
W_{k}\left(x ; Z^{L}\right]=\boldsymbol{W}_{k}\left(Z^{\ell}(x)\right) .
$$

It is easy to see that the expression (7.17) solves the Heisenberg equations of motion (7.16). In this roundabout way, we recover the canonical counterpart of the spacetime equation (2.18). 
Now impose the condition that the dust does not rotate, so that its vorticity (7.4) vanishes. This implies that (locally) $W_{k}(x) Z^{k}{ }_{a}(x)$ must be a gradient of a spatial scalar. Therefore the function $\boldsymbol{W}_{k}\left(z^{\ell}\right)$ in Eq. (7.17) must be the gradient of a function $-\Theta\left(z^{\ell}\right)$ :

$$
W_{k}\left(x ; Z^{L}\right]=-\Theta_{, k}\left(Z^{\ell}(x)\right) .
$$

The condition (7.18) enables us to transform to zero the particular solution (7.17) of the Heisenberg equations of motion by successive applications of the symmetry transformation (3.27) generated by the Noether charge $Q[\boldsymbol{\vartheta}]$. This transforms the momentum $P_{k}(x)=$ $-P(x) W_{k}(x)$ to zero.

To summarize, any particular solution (7.18) of the constraint equations and the Hamilton equations of motion of non-rotating dust can be cast into the form in which the dust time foliation $\mathcal{T}_{\perp}$ is orthogonal to the dust frame $\mathcal{S}$. When we demand from the outset not only that the dust be non-rotating, but also that the time foliation $\mathcal{T}$ which is used for its description be orthogonal to the dust frame, we can impose the additional constraint

$$
P_{k}(x)=0 .
$$

This constraint is stronger than the requirement (7.10) that the vorticity $\Omega_{a b}(x)$ vanish; Eq. (7.19) implies Eq. (7.10), while Eq. (7.10) does not automatically enforce Eq. (7.19).

Now consider the system of constraints $H_{\uparrow}(x)=0, H_{\uparrow k}(x)=0$, and $P_{k}(x)=0$. This constraint system is first class: In Sec. 4 we showed that the new super-Hamiltonian constraints $H_{\uparrow}(x)$ and the new supermomentum constraints $H_{\uparrow k}(x)$ have vanishing Poisson brackets among themselves. The momenta $P_{k}(x)$ also have vanishing Poisson brackets among themselves and with the generators $H_{\uparrow}(x)$. From Eq. (4.9) we see that the Poisson brackets of $P_{k}$ and $H_{\uparrow k}$ can be written as

$$
\left\{P_{k}(x), H_{\uparrow \ell}\left(x^{\prime}\right)\right\}=Z_{\ell}^{a}\left(x^{\prime}\right)\left\{P_{k}(x), H_{a}\left(x^{\prime}\right)\right\}+\left\{P_{k}(x), Z_{\ell}^{a}\left(x^{\prime}\right)\right\} H_{a}\left(x^{\prime}\right) .
$$

Because $P_{k}(x)$ are scalars under Diff $\Sigma$, their Poisson brackets with $H_{a}(x)$ are linear in $P_{k}(x)$. Thus, the Poisson brackets $\left\{P_{k}(x), H_{\uparrow \ell}\left(x^{\prime}\right)\right\}$ also close.

In general, the functional $Q[\boldsymbol{\vartheta}]$ from Eq. (3.25) does not have vanishing Poisson brackets with $P_{k}(x)$, and thus does not generate a symmetry of the theory defined by the new system of constraints $H_{\uparrow}=0, H_{\uparrow k}=0$, and $P_{k}=0$. Indeed, for $\boldsymbol{\vartheta}_{, k} \neq 0, Q[\boldsymbol{\vartheta}]$ generates a tilt of the time foliation $\mathcal{T}$ that spoils the Gaussian character of the coordinate system $\left(\tau, z^{k}\right)$ based on the irrotational dust. For $\boldsymbol{\vartheta}=$ const, $Q[\boldsymbol{\vartheta}]$ generates a symmetry that consists of a relabeling of the leaves of the privileged foliation $\mathcal{T}_{\perp}$. In this case, $Q[\boldsymbol{\vartheta}]$ is a conserved charge whose value (for $\boldsymbol{\vartheta}=1$ ) is the total relativistic mass of the dust. From Eq. (3.24), the functional $Q[\overrightarrow{\boldsymbol{\xi}}]$ is seen to equal the new constraint (7.19) smeared with a prescribed dust space vector field $-\boldsymbol{\xi}^{k}(Z(x))$. Thus, the Poisson brackets of $Q[\overrightarrow{\boldsymbol{\xi}}]$ with $P_{k}(x)$ vanish weakly. The smeared constraint $Q[\overrightarrow{\boldsymbol{\xi}}]$ is the canonical generator of dust space diffeomorphisms, in which the Lagrangian coordinate labels for the dust particles are changed.

The additional constraint (7.19) enables us to replace the Hamiltonian constraint $H_{\uparrow}$ by an equivalent constraint that does not involve the square of the gravitational superHamiltonian $H_{\perp}^{G}$. Modulo Eq. (7.19), the new supermomentum constraint (4.9) implies $H_{a}^{G} \approx-\sqrt{G} T_{, a}$. This can be combined with the definition $G:=\left(H_{\perp}^{G}\right)^{2}-g^{a b} H_{a}^{G} H_{b}^{G}$ to yield $\left(H_{\perp}^{G}\right)^{2}=\left(1+g^{a b} T_{, a} T_{, b}\right) G$. The Hamiltonian constraint $H_{\uparrow}=P-\sqrt{G}$ then takes the form 


$$
H_{\uparrow 0}:=P(x)+h_{0}(x ; T, g, p]=0,
$$

with the Hamiltonian

$$
h_{0}(x ; T, g, p]:=\left(1+g^{a b}(x) T_{, a}(x) T_{, b}(x)\right)^{-1 / 2} H_{\perp}^{G}(x) .
$$

The new Hamiltonian (7.22) does not contain an inconvenient square root and is quadratic in the gravitational momentum $p^{a b}(x)$. Unlike the general Hamiltonian $h(x)$ of Eq. (4.7), however, it does depend on the Gaussian time $T(x)$ labeling the leaves of $\mathcal{T}_{\perp}$. The constraint (7.21) was employed for the quantization of gravity interacting with non-rotating dust in our previous work [10].

\section{Quantizing a Non-Rotating Dust}

We have seen that any particular solution of the classical constraint equations and the Hamilton equations of motion for non-rotating dust can be described with respect to the time foliation $\mathcal{T}_{\perp}$ that is orthogonal to the dust frame $Z^{k}{ }_{, \alpha}(y)$. By imposing the constraint $P_{k}(x)=0$, we demand both that the dust be non-rotating and that its motion be described with respect to the frame-orthogonal foliation. The canonical description of this system can be formulated either in terms of the original canonical variables or in terms of the new canonical dust-space variables. For the original variables, the new constraint has the simple form $P_{k}(x)=0$, while the supermomentum constraint has a relatively complicated form (4.9). With the dust-space variables, the supermomentum constraint is simple - it is the momentum conjugate to $Z^{k}(x)$-while the new constraint (7.19) takes the form

$$
P_{k}(x)=H_{\uparrow k}(x)-\left.\left|\frac{\partial Z(x)}{\partial x}\right|\left(\boldsymbol{H}_{k}^{G}(z)+\boldsymbol{P}(z) \boldsymbol{T}_{, k}(z)\right)\right|_{z=Z(x)} .
$$

In each case, the constraint that generates diffeomorphisms of the manifold on which the tensor fields live $(\Sigma$ or $\mathcal{S}$ ) has a relatively complicated form, while the constraint that generates diffeomorphisms of the complementary manifold is trivial. Thus, we see that for non-rotating dust with the frame-orthogonal foliation there is no relative advantage in using one set of canonical variables as opposed to the other. In what follows we will work with the original canonical variables.

The system of constraints $H_{\uparrow k}=0, H_{\uparrow 0}=0$, and $P_{k}=0$ is first class. Therefore these constraints can be imposed consistently as restrictions on the state functional $\Psi[Z, T, g]$. The constraint (7.19) in particular yields the restriction

$$
\hat{P}_{k}(x) \Psi[Z, T, g]=0,
$$

and implies that the state $\Psi$ does not depend on the frame variables $Z^{k}(x)$. Our task then is to find solutions $\Psi[T, g]$ of the operator equations that follow from the classical constraints $H_{\uparrow 0}(x)=0$ and $H_{\uparrow k}(x)=0$. These are the many-fingered time Schrödinger equation

$$
\hat{H}_{\uparrow 0}(x) \Psi[T, g]=0 \quad \Longleftrightarrow \quad i \frac{\delta \Psi[T, g]}{\delta T(x)}=h_{0}(x ; T, \hat{g}, \hat{p}] \Psi[T, g],
$$


and the equation that requires $\Psi$ to be invariant under Diff $\Sigma$,

$$
\left(T_{, a}(x) \hat{P}(x)+\hat{H}_{a}^{G}(x)\right) \Psi[T, g]=0 .
$$

Functionals $\Psi[T, g]$ that satisfy Eqs. (7.25) and (7.26) describe the quantum states of nonrotating dust with respect to the frame-orthogonal time foliation $\mathcal{T}_{\perp}$. We have explored this scheme in a previous paper [10].

One might expect that any state $\Psi$ with vanishing vorticity,

$$
\hat{\Omega}_{a b}(x) \Psi[Z, T, g]=0,
$$

can be transformed into a state satisfying Eq. (7.24) by the action of the symmetry operator (6.25). However, this is not so. The underlying reason is that a classical $P_{k}(x)$ can be brought to zero by a symmetry transformation for any particular solution of the equations describing a non-rotating dust, but the state functional $\Psi[Z, T, g]$ generally describes an ensemble of quantum systems corresponding to a superposition of such individual solutions. Correspondingly, there is in general no single function $\Theta(Z(x))$ whose charge $\hat{Q}[\boldsymbol{\Theta}]$ would turn a state functional that satisfies Eq. (7.27) into one that satisfies the stronger equation (7.24).

We now ask whether every $Z$-independent solution $\Psi[T, g]$ of Eqs. (6.47) is also a solution of Eqs. (7.25) and (7.26), and inversely, whether every solution of Eqs. (7.25) and (7.26) is also a solution of Eqs. (6.47). The proof of such a statement stumbles over a factor ordering problem. Assume that $\Psi[T, g]$ solves Eq. (6.47a) and hence also the iterated equation

$$
\hat{P}^{2}(x) \Psi[T, g]=G(x ; \hat{g}, \hat{p}] \Psi[T, g] .
$$

If the operator $\hat{G}(x)$ were factor ordered as

$$
G(x ; \hat{g}, \hat{p}]=\left(\hat{H}_{\perp}^{G}(x)\right)^{2}-\hat{g}^{a b}(x) \hat{H}_{a}^{G}(x) \hat{H}_{b}^{G}(x),
$$

then its application on a state $\Psi[T, g]$ which also satisfies Eq. (6.47b) would yield

$$
\left(1+\hat{g}^{a b}(x) T_{, a}(x) T_{, b}(x)\right) \hat{P}^{2}(x) \Psi=\left(\hat{H}_{\perp}^{G}(x)\right)^{2} \Psi .
$$

The state $\Psi$ would then satisfy Eqs. (7.25) and (7.26) with the operator $\hat{h}_{0}(x)$ factor ordered as

$$
h_{0}(x ; T, \hat{g}, \hat{p}]=\left(1+\hat{g}^{a b}(x) T_{, a}(x) T_{, b}(x)\right)^{-1} \hat{H}_{\perp}^{G}(x) .
$$

However, the factor ordering of the operator $G(x ; \hat{g}, \hat{p}]$ is dictated by the requirement that the operators $\hat{G}(x)$ and $\hat{G}\left(x^{\prime}\right)$ commute, thereby replicating the vanishing of the corresponding classical Poisson brackets, Eq. (4.22). One can hardly expect that any ordering of the factors $\hat{g}$ and $\hat{p}$ in the individual operators $H_{\perp}^{G}(x ; \hat{g}, \hat{p}]$ and $H_{a}^{G}(x ; \hat{g}, \hat{p}]$, followed by the ordering (7.29) of $\hat{G}(x)$, can achieve this aim. Similarly, one cannot expect that the ordering (7.31) would yield Hamiltonian densities $\hat{h}_{0}(x)$ that are self-adjoint.

Formal considerations like these indicate that, even if we were able to construct consistent quantum field theories based respectively on the Schrödinger equations (6.47a) and (7.25), these theories would likely be non-equivalent. 


\section{ACKNOWLEDGMENTS}

The work on this paper was partially supported by the NSF grant PHY-9207225 to the University of Utah.

\section{APPENDIX A: NOTATION}

Manifolds. Manifolds play an important role in our study: spacetime $\mathcal{M}$, space $\Sigma$, dust space $\mathcal{S}$, and dust time $\mathcal{T}$. Points $y \in \mathcal{M}$ are labeled by local coordinates $y^{\alpha}$ which carry Greek indices. Points $x \in \Sigma$ are labeled by local coordinates $x^{a}$ with indices from the beginning of the Latin alphabet. Points $z \in \mathcal{S}$ are labeled by local coordinates $z^{k}$ with indices from the middle of the Latin alphabet. Instants $\tau \in \mathcal{T}$ are always labeled by the Cartesian coordinate which (a minor ambiguity) is also called $\tau$.

Mappings. Geometric properties of spacetime and physical properties of the dust are studied in the canonical formalism on (spacelike) embeddings $Y: \Sigma \rightarrow \mathcal{M}$ by $x \in \Sigma \mapsto y=$ $Y(x) \in \mathcal{M}$. The spacetime metric $\gamma$ on $\mathcal{M}$ is pulled back by the embedding mapping $Y$ into the spatial metric $g$ on $\Sigma$. The mapping $\Upsilon: \mathcal{T} \times \mathcal{S} \rightarrow \mathcal{M}$ by $(\tau \in \mathcal{T}, z \in \mathcal{S}) \mapsto y=$ $\Upsilon(\tau, z) \in \mathcal{M}$ foliates $\mathcal{M}$ into the dust time $\mathcal{T}$ and the dust space $\mathcal{S}$. The inverse mappings are $T: \mathcal{M} \rightarrow \mathcal{T}$ by $y \in \mathcal{M} \mapsto \tau=T(y) \in \mathcal{T}$ and $Z: \mathcal{M} \rightarrow \mathcal{S}$ by $y \in \mathcal{M} \mapsto z=Z(y) \in \mathcal{S}$. A point $x \in \Sigma$ of the embedded hypersurface $Y(\Sigma)$ lies at the instant $\tau=T \circ Y(x)$ of the dust time and at the dust space point $z=Z \circ Y(x)$. The proliferation of symbols is avoided by a slight misuse of notation: the mappings $T \circ Y: \Sigma \rightarrow \mathcal{T}$ and $Z \circ Y: \Sigma \rightarrow \mathcal{S}$ are simply called $T$ and $Z$. We thus write $T: \Sigma \rightarrow \mathcal{T}$ by $x \in \Sigma \mapsto \tau=T(x) \in \mathcal{T}$ and $Z: \Sigma \rightarrow \mathcal{S}$ by $x \in \Sigma \mapsto z=Z(x) \in \mathcal{S}$. It is fairly clear from the context what mappings the symbols $T$ and $Z$ denote.

The mapping $Z: \Sigma \rightarrow \mathcal{S}$ has the inverse $X=Z^{-1}: \mathcal{S} \rightarrow \Sigma$ by $z \in \mathcal{S} \mapsto x=X(z) \in \Sigma$. Tensor fields on $\Sigma$ are mapped to $\mathcal{S}$ by $Z^{*}$, and tensor fields on $\mathcal{S}$ are mapped to $\Sigma$ by $X^{*}$. The corresponding tensor fields are given lightface kernel symbols on $\Sigma$ and boldface kernel symbols on $\mathcal{S}$. For example, the spatial metric $g_{a b}(x)$ on $\Sigma$ is mapped to $\boldsymbol{g}_{k \ell}(z)$ on $\mathcal{S}$ and the dust time $T(x)$ on $\Sigma$ is mapped to $\boldsymbol{T}(z)$ on $\mathcal{S}$.

Notice that in all these examples, the mappings are denoted by capital letters, and their values by the corresponding lower case letters.

Derivatives. Partial derivatives are denoted by comma followed by the appropriate coordinate index: ${ }_{, \alpha},{ }_{, a}$ and,$k$. The covariant derivative on $(\mathcal{M}, \gamma)$ is written as $\nabla_{\alpha}$, the covariant derivative on $(\Sigma, g)$ as $D_{a}$. The pound symbol $£$ is used for the Lie derivative.

Brackets. The arguments of functions are enclosed in round brackets ( ), the arguments of functionals in square brackets [ ]. Mixed brackets ( ] indicate that an object is a function of one argument and a functional of another: e.g., $R(x ; g]$ means that the scalar curvature $R$ on $\Sigma$ is a function of $x \in \Sigma$ and a functional of the induced metric $g_{a b}\left(x^{\prime}\right)$.

The Lie brackets of vector fields and the commutators of operators are denoted by square brackets $[$,$] . Braces \{$,$\} are reserved for the Poisson brackets.$

Densities and operators. When the same quantity appears both as a tensor and as

a tensor density, the density is marked by a tilde. Thus, $J^{\alpha}$ is a vector while $\widetilde{J}^{\alpha}$ is a vector density of weight 1 . All operators wear hats, as $\hat{g}(x)$ or $\hat{p}(x)$. 
Units. We are using natural units in which the speed of light $c$ and the rationalized Newton's constant $16 \pi G$ are set equal to $1: c=1=16 \pi G$. In these units, the gravitational Lagrangian is simply the densitized scalar curvature $|\gamma|^{1 / 2} R(y ; \gamma]$, and the Einstein law of gravitation takes the form $R^{\alpha \beta}-\frac{1}{2} R \gamma^{\alpha \beta}=\frac{1}{2} T^{\alpha \beta}$.

\section{APPENDIX B: ALTERNATIVE FORMS OF THE SPACETIME ACTION}

The action (2.4) is a functional of eight state variables, $T, Z^{k}$ and $M, W_{k}$. It can be cast into an equivalent form that depends on fewer state variables by solving the Euler equation (2.7), obtained by varying $W_{k}$, with respect to $W_{k}$. This yields

$$
W_{k}=h_{k \ell} Z_{, \alpha}^{\ell} \gamma^{\alpha \beta} T_{, \beta}
$$

where $h_{k \ell}$ is the inverse of the matrix

$$
h^{k \ell}:=Z_{, \alpha}^{k} \gamma^{\alpha \beta} Z_{, \beta}^{\ell} .
$$

The metric $h_{k \ell}$ measures the distance $d s$ orthogonal to the flow lines between neighboring flow lines with Lagrangian coordinates $z^{k}$ and $z^{k}+d z^{k}$ :

$$
d s^{2}=h_{k \ell} d z^{k} d z^{\ell}
$$

By using Eq. (B.1) to eliminate $W_{k}$ from the action, we obtain the equivalent action

$$
S^{D}\left[T, Z^{k} ; M ; \gamma_{\alpha \beta}\right]:=S^{D}\left[T, Z^{k} ; M, W_{k}=W_{k}\left[T, Z^{\ell}, \gamma_{\alpha \beta}\right] ; \gamma_{\alpha \beta}\right]
$$

which depends on the four dynamical variables $T, Z^{k}$, and a single multiplier $M$.

The mass multiplier cannot be eliminated at the spacetime level by an algebraic process, because the equation (2.6) obtained by varying $M$ does not depend on $M$. In Sec. 3a we showed how $M$ can be eliminated from the canonical action (or, rather, how $M$ can be replaced by the canonical momentum conjugate to the time function $T$ ). Instead of decreasing the number of variables by solving the field equations, one can increase the number of variables by adjoining valid equations to the action by additional Lagrange multipliers. As an example, the definition (2.2) of $U_{\alpha}$ can be adjoined to the action (2.4) by a set of four multipliers $\widetilde{J}^{\alpha}$ that are densities of weight 1 . One obtains thereby the action

$$
\begin{aligned}
& S^{D}\left[T, Z^{k} ; M, W_{k} ; U_{\alpha}, \widetilde{J}^{\alpha} ; \gamma_{\alpha \beta}\right] \\
& \quad=\int_{\mathcal{M}} d^{4} y\left(-\frac{1}{2}|\gamma|^{1 / 2} M\left(\gamma^{\alpha \beta} U_{\alpha} U_{\beta}+1\right)+\widetilde{J}^{\alpha}\left(U_{\alpha}+T_{, \alpha}-W_{k} Z^{k}{ }_{, \alpha}\right)\right)
\end{aligned}
$$

which depends on sixteen state variables $T, Z^{k}, M, W_{k}$ and $U_{\alpha}, \widetilde{J}^{\alpha}$. By varying the action (B.5) with respect to $\widetilde{J}^{\alpha}$, we recover the definition (2.2) of $U_{\alpha}$. By varying $U_{\alpha}$, we obtain

$$
\widetilde{J}^{\alpha}-|\gamma|^{1 / 2} M U^{\alpha}=0
$$


This shows that the multipliers $\widetilde{J}^{\alpha}$ are the components of the (densitized) mass current (2.15). By calculating $U^{\alpha}$ from Eq. (B.6),

$$
U^{\alpha}=M^{-1}|\gamma|^{-1 / 2} \widetilde{J}^{\alpha}
$$

and substituting it back into the action (B.5), we obtain an equivalent action

$$
\begin{aligned}
S^{D} & {\left[T, Z^{k} ; M, W_{k} ; \widetilde{J}^{\alpha} ; \gamma_{\alpha \beta}\right] } \\
& =\int_{\mathcal{M}} d^{4} y\left(\frac{1}{2}|\gamma|^{-1 / 2} M^{-1} \gamma_{\alpha \beta} \widetilde{J}^{\alpha} \widetilde{J}^{\beta}-\frac{1}{2}|\gamma|^{1 / 2} M+\widetilde{J}^{\alpha}\left(T_{, \alpha}-W_{k} Z^{k}{ }_{, \alpha}\right)\right)
\end{aligned}
$$

that depends on twelve state variables $T, Z^{k}, M, W_{k}$ and $\widetilde{J}^{\alpha}$. The variation of the action (B.8) with respect to $\mathrm{M}$ yields an equation that determines $\mathrm{M}$, namely,

$$
M=|\gamma|^{-1 / 2} \sqrt{-\gamma_{\alpha \beta} \widetilde{J}^{\alpha} \tilde{J}^{\beta}}
$$

By using Eq. (B.9), M can be eliminated from the action (B.8). The result is the action

$$
\begin{aligned}
S^{D}\left[T, Z^{k} ;\right. & \left.W_{k} ; \tilde{J}^{\alpha} ; \gamma_{\alpha \beta}\right] \\
& =\int_{\mathcal{M}} d^{4} y\left(-\sqrt{-\gamma_{\alpha \beta} \widetilde{J}^{\alpha} \widetilde{J}^{\beta}}+\widetilde{J}^{\alpha}\left(T_{, \alpha}-W_{k} Z_{, \alpha}^{k}\right)\right)
\end{aligned}
$$

that depends on eleven state variables $T, Z^{k}, W_{k}$ and $\widetilde{J}^{\alpha}$. This action for dust was introduced in Ref [26] in the study of perfect fluid actions.

All of the actions (2.4), (B.4), (B.5), (B.8), and (B.10) lead to the same canonical action. We have chosen the action (2.4) because the number of state variables exactly matches the number of canonical coordinates plus conjugate momenta. The physical meaning of the momenta then clearly emerges from the Legendre transformation.

\section{APPENDIX C: SYMMETRY TRANSFORMATIONS}

Transformations of the dust variables $\left(T, Z^{k}, W_{k}\right) \rightarrow\left(T^{\prime}, Z^{k^{\prime}}, W_{k^{\prime}}\right)$ that satisfy

$$
S^{D}\left[T^{\prime}\left(T, Z^{\ell}, W_{\ell}\right), Z^{k^{\prime}}\left(T, Z^{\ell}, W_{\ell}\right), W_{k^{\prime}}\left(T, Z^{\ell}, W_{\ell}\right)\right]=S^{D}\left[T, Z^{k}, W_{k}\right]
$$

constitute symmetries of the dust action. Inspection of the action (2.4)-(2.5) shows that transformations that preserve the Pfaff form (2.3),

$$
-d T+W_{k} d Z^{k}=-d T^{\prime}+W_{k^{\prime}} d Z^{k^{\prime}}
$$

are symmetries. A Pfaff form $-d T+W_{k} d Z^{k}$, with $k=1,2,3$, always can be reduced to $d A+B d C$ for some functions $A, B$, and $C$ [18]; however, here we are interested in invertible 
transformations that leave the action functional invariant, as expressed though Eq. (C.1). (See Refs. 26, 27] for discussions of the use of the reduced Pfaff form in the construction of perfect fluid action functionals.) We shall confine our attention to transformations that are ultralocal in spacetime, so the primed variables at a given spacetime point $y$ depend only on the unprimed variables at the point $y$. In particular, the primed variables are not allowed to depend on derivatives of the unprimed variables.

For the case of ultralocal transformations, the relationship (C.2) can be written as

$$
W_{k^{\prime}}\left(T, Z^{\ell}, W_{\ell}\right) d Z^{k^{\prime}}\left(T, Z^{\ell}, W_{\ell}\right)=W_{k} d Z^{k}+d \mathcal{F}\left(T, Z^{\ell}, W_{\ell}\right)
$$

and viewed as a time $T$-dependent canonical transformation for a fictitious phase space with coordinates $Z^{k}$ and momenta $W_{k}$ [26,27]. Here, $\mathcal{F}$ is the function of $T$ and of the 'canonical variables' $Z^{k}, W_{k}$, defined by $\mathcal{F}:=T^{\prime}-T$. Now, for those transformations that are connected to the identity, the dependence $Z^{k^{\prime}}=Z^{k^{\prime}}\left(T, Z^{\ell}, W_{\ell}\right)$ of the primed 'coordinates' on the unprimed variables can be inverted for the unprimed 'coordinates', $Z^{k}=Z^{k}\left(T, Z^{\ell^{\prime}}, W_{\ell}\right)$. Then $T, Z^{k^{\prime}}$, and $W_{k}$ can be treated as independent variables by substituting the functional relationship $Z^{k}=Z^{k}\left(T, Z^{\ell^{\prime}}, W_{\ell}\right)$ into Eq. (C.3). This leads to the expression

$$
W_{k^{\prime}}\left(T, Z^{\ell^{\prime}}, W_{\ell}\right) d Z^{k^{\prime}}=W_{k} d Z^{k}\left(T, Z^{\ell^{\prime}}, W_{\ell}\right)+d \mathcal{F}\left(T, Z^{\ell^{\prime}}, W_{\ell}\right),
$$

with a slight abuse of notation in which $W_{k^{\prime}}\left(T, Z^{\ell}\left(T, Z^{m^{\prime}}, W_{m}\right), W_{\ell}\right)$ is set equal to $W_{k^{\prime}}\left(T, Z^{\ell^{\prime}}, W_{\ell}\right)$, and likewise for $\mathcal{F}$. In terms of the generating function $F\left(T, Z^{\ell^{\prime}}, W_{\ell}\right):=$ $\mathcal{F}\left(T, Z^{\ell^{\prime}}, W_{\ell}\right)+W_{k} Z^{k}\left(T, Z^{\ell^{\prime}}, W_{\ell}\right)$, Eq. (C.4) can be rewritten as

$$
W_{k^{\prime}}\left(T, Z^{\ell^{\prime}}, W_{\ell}\right) d Z^{k^{\prime}}+Z^{k}\left(T, Z^{\ell^{\prime}}, W_{\ell}\right) d W_{k}=d F\left(T, Z^{\ell^{\prime}}, W_{\ell}\right) .
$$

This expression along with the definitions of $\mathcal{F}$ and $F$ yield the results

$$
\begin{aligned}
T^{\prime}-T & =F-W_{k} \frac{\partial F}{\partial W_{k}}, \\
Z^{k} & =\frac{\partial F}{\partial W_{k}}, \\
W_{k^{\prime}} & =\frac{\partial F}{\partial Z^{k^{\prime}}},
\end{aligned}
$$

where $F=F\left(Z^{\ell^{\prime}}, W_{\ell}\right)$ is independent of $T$.

The Noether currents (2.29) associated with one-parameter subgroups of symmetries $F\left(Z^{\ell^{\prime}}, W_{\ell}, \sigma\right)$ connected to the identity $F\left(Z^{\ell^{\prime}}, W_{\ell}, 0\right)=Z^{k^{\prime}} W_{k}$ are given by

$$
\widetilde{J}^{\alpha}[f]=|\gamma|^{1 / 2} J^{\alpha} f\left(Z^{\ell}, W_{\ell}\right),
$$

where $J^{\alpha}$ is the mass current (2.15) and

$$
f\left(Z^{\ell}, W_{\ell}\right):=\left.\frac{\partial F\left(Z^{\ell^{\prime}}, W_{\ell}, \sigma\right)}{\partial \sigma}\right|_{\sigma=0} .
$$


The corresponding Noether charges (2.28) can be written as

$$
\begin{aligned}
Q[f] & =-\int_{\Sigma} d^{3} x|g|^{1 / 2} n_{\alpha} J^{\alpha} f\left(Z^{\ell}, W_{\ell}\right) \\
& =\int_{\Sigma} d^{3} x P f\left(Z^{\ell},-P_{\ell} / P\right) .
\end{aligned}
$$

In expression (C.9b), we have made use of the canonical variables $P$ and $P_{k}$ as defined by Eqs. (3.10) and (3.12).

In Sec. 2e of the main text we restrict our attention to those symmetry transformations that mix the dust spacetime variables $T$ and $Z^{k}$ with one another. That is, we consider transformations for which $T^{\prime}$ and $Z^{k^{\prime}}$ are functions of $T$ and $Z^{k}$ only. If this restriction is imposed on the transformations (C.6), it follows that the most general generating function $F$ has the form

$$
F\left(Z^{\ell^{\prime}}, W_{\ell}\right)=W_{k} \boldsymbol{\Xi}^{k}\left(Z^{\ell^{\prime}}\right)+\boldsymbol{\Theta}\left(\boldsymbol{\Xi}^{\ell}\left(Z^{m^{\prime}}\right)\right)
$$

The corresponding symmetry transformations (C.6) are given by

$$
\begin{aligned}
T^{\prime} & =T+\boldsymbol{\Theta}\left(Z^{m}\right), \\
Z^{k} & =\boldsymbol{\Xi}^{k}\left(Z^{m^{\prime}}\right), \\
W_{k^{\prime}} & =\boldsymbol{\Xi}_{, k^{\prime}}^{\ell}\left(Z^{m^{\prime}}\right)\left(W_{\ell}+\boldsymbol{\Theta}_{, \ell}\left(Z^{m}\right)\right),
\end{aligned}
$$

which coincide with the transformations (2.26)-(2.27) discussed in Sec. 2e.

The Lie algebra of one-parameter groups of symmetries generated by $F$ of the form (C.10) is labeled by the functions

$$
f\left(Z^{\ell}, W_{\ell}\right):=\left.\frac{\partial F\left(Z^{\ell^{\prime}}, W_{\ell}, \sigma\right)}{\partial \sigma}\right|_{\sigma=0}=W_{k} \boldsymbol{\xi}^{k}\left(Z^{\ell}\right)+\boldsymbol{\vartheta}\left(Z^{\ell}\right),
$$

where $\boldsymbol{\xi}^{k}\left(Z^{\ell}\right)$ and $\boldsymbol{\vartheta}\left(Z^{\ell}\right)$ are defined in Eq. (2.32). Insertion of this expression for $f$ into the Noether charge (C.9) yields

$$
\begin{aligned}
Q[\boldsymbol{\xi}, \boldsymbol{\vartheta}] & =-\int_{\Sigma} d^{3} x|g|^{1 / 2} n_{\alpha}\left(J_{k}^{\alpha} \boldsymbol{\xi}^{k}\left(Z^{\ell}\right)+J^{\alpha} \boldsymbol{\vartheta}\left(Z^{\ell}\right)\right) \\
& =\int_{\Sigma} d^{3} x\left(-P_{k} \boldsymbol{\xi}^{k}\left(Z^{\ell}\right)+P \boldsymbol{\vartheta}\left(Z^{\ell}\right)\right)
\end{aligned}
$$

where $J^{\alpha}$ and $J_{k}^{\alpha}$ are the mass current (2.15) and the momentum current (2.16), respectively. The charges $Q[\boldsymbol{\xi}, \boldsymbol{\vartheta}]$ coincide with the Noether charges (2.36)-(2.37) and (3.24)-(3.25) presented in the main text.

\section{APPENDIX D: THE ACTION FOR RELATIVISTIC PARTICLES}

The geodesic motion of a single relativistic particle of rest mass $m$ is generated by the super-Hamiltonian 


$$
H:=\frac{1}{2 m}\left(\gamma^{\alpha \beta}(y) P_{\alpha} P_{\beta}+m^{2}\right)
$$

The spacetime coordinates $y^{\alpha}$ of the particle and the four-momentum $P_{\alpha}$ are canonically conjugate variables. These variables can be split into their space and time components with respect to the foliation (3.1)-(3.2). The momenta conjugate to $t$ and $x^{a}$ are

$$
p_{t}:=\dot{X}^{\alpha} P_{\alpha} \quad \text { and } \quad p_{a}:=X_{, a}^{\alpha} P_{\alpha} .
$$

By using Eqs. (3.3) and (3.4), the super-Hamiltonian constraint $H=0$ can be resolved with respect to the energy variable $-p_{t}$ :

$$
\begin{aligned}
-p_{t} & =h\left(t, x^{a}, p_{a}\right) \\
& =-N^{a}(t, x) p_{a}+N^{\perp}(t, x) \sqrt{m^{2}+g^{a b}(t, x) p_{a} p_{b}} .
\end{aligned}
$$

The expression $h\left(t, x^{a}, p_{a}\right)$ is the Hamiltonian of the particle.

The action associated with the Hamiltonian (D.3) is

$$
S\left[x^{a}, p_{a}\right]=\int d t L\left(x^{a}, p_{a}, \dot{x}^{a}\right)=\int d t\left(p_{a} \frac{d x^{a}}{d t}-h\left(t, x^{a}, p_{a}\right)\right) .
$$

Observe that the derivative of the canonical Lagrangian $L$ with respect to mass $m$ is

$$
\frac{\partial L}{\partial m}=-\frac{N^{\perp}}{\sqrt{1+g^{a b} p_{a} p_{b} / m^{2}}} .
$$

Since $N^{\perp} d t$ is the orthogonal proper time separation between $t=$ const surfaces, and $\sqrt{1+g^{a b} p_{a} p_{b} / m^{2}}$ is the relativistic 'gamma factor', $\partial L / \partial m$ equals the ratio $d \tau / d t$, where $\tau$ is the proper time along the particle worldline. This shows that the action (D.4) can be extended to a functional of $m(t)$ and $\tau(t)$,

$$
\begin{aligned}
S\left[\tau, m ; x^{a}, p_{a}\right]=\int d t & \left(m \frac{d \tau}{d t}+p_{a} \frac{d x^{a}}{d t}-N^{a}(t, x)\left(-p_{a}\right)\right. \\
& \left.-N^{\perp}(t, x) \sqrt{m^{2}+g^{a b}(t, x) p_{a} p_{b}}\right) .
\end{aligned}
$$

Extremization of $S$ with respect to $m$ relates $\partial L / \partial m$ to $d \tau / d t$. Extremization of $S$ with respect to $\tau$ yields the condition $d m / d t=0$, that the particle mass is a constant of motion.

We now compare the relativistic particle action (D.6) with the canonical dust action (3.16). For the purpose of comparison, the metric tensor components $N^{\perp}(t, x), N^{a}(t, x)$, $g_{a b}(t, x)$ are treated as prescribed external fields. From the analysis of Sec. 5 it follows that the dust action (3.16) can be written as a functional of canonical variables $\boldsymbol{T}(z), \boldsymbol{P}(z)$, $Z^{k}(x)$, and $H_{\uparrow k}(x):=Z_{k}^{a}(x) H_{a}^{D}(x)$. We can make a further canonical transformation [15] by replacing the dust frame $Z^{k}(x)$ with its inverse, $X^{a}(z)$. The new variable $X^{a}(z)$ specifies the spatial location of the dust particle with Lagrangian coordinate label $z^{k}$. The momentum conjugate to $X^{a}(z)$ is found from the relationship 


$$
\int_{\Sigma} d^{3} x H_{\uparrow k}(x) \dot{Z}^{k}(x)=-\int_{\mathcal{S}} d^{3} z\left|\frac{\partial X(z)}{\partial z}\right| H_{\uparrow k}(X(z)) Z_{, a}^{k}(X(z)) \dot{X}^{a}(z) .
$$

With the momentum conjugate to $X^{a}(z)$ denoted by $P_{a}(z)$, the action (3.16) becomes

$$
\begin{aligned}
S^{D}\left[X^{a}, P_{a} ; \boldsymbol{T}, \boldsymbol{P}\right]=\int_{\mathbb{R}} d t \int_{S} d^{3} z & \left(\boldsymbol{P}(z) \dot{\boldsymbol{T}}(z)+P_{a}(z) \dot{X}^{a}(z)-N^{a}(t, X(z))\left(-P_{a}(z)\right)\right. \\
& \left.-N^{\perp}(t, X(z)) \sqrt{\boldsymbol{P}^{2}(z)+g^{a b}(t, X(z)) P_{a}(z) P_{b}(z)}\right) .
\end{aligned}
$$

This action coincides with the relativistic particle action in the following sense. The action for many relativistic particles is obtained from the single particle action (D.6) by summing over the individual particles. With the particles labeled by $z=1,2, \ldots$, the canonical variables are given by the set $\left(\tau_{z}, m_{z}, x_{z}^{a}, p_{z a}\right)$. In the continuum limit of an infinite number of particles scattered throughout space, the sum $\sum_{z}$ over individual particles is replaced by an integral $\int d^{3} z$ over the space of particle labels, the dust space $\mathcal{S}$. At the same time, the canonical momenta $m_{z}$ and $p_{z a}$ become densities in the space of labels. The resulting action functional is precisely the dust action (D.8), with the correspondences $\tau_{z} \rightarrow \boldsymbol{T}(z)$, $m_{z} \rightarrow \boldsymbol{P}(z) d^{3} z, x_{z}^{a} \rightarrow X^{a}(z)$, and $p_{z a} \rightarrow P_{a}(z) d^{3} z$.

\section{APPENDIX E: THE POISSON ALGEBRA OF $G(x)$}

We check by direct calculation that the quadratic combinations $G(x)$, Eq. (4.7b), of the gravitational super-Hamiltonian $H_{\perp}^{G}(x)$ and supermomentum $H_{a}^{G}(x)$ have strongly vanishing Poisson brackets, Eq. (4.22). The result follows from the fact that the gravitational generators $H_{\perp}^{G}(x)$ and $H_{a}^{G}(x)$ satisfy the Dirac 'algebra' Eqs. (4.1)-(4.3), and from the action of these generators on the metric $g^{a b}(x)$.

Let us first show that the expressions

$$
F(x):=g^{a b}(x) H_{a}^{G}(x) H_{b}^{G}(x)
$$

commute among themselves,

$$
\left\{F(x), F\left(x^{\prime}\right)\right\}=0 \text {. }
$$

From the definition (E.1), it holds that

$$
\begin{aligned}
\left\{F(x), F\left(x^{\prime}\right)\right\}=( & \left.2\left\{F(x), H_{a}^{G}\left(x^{\prime}\right)\right\} H^{G a}\left(x^{\prime}\right)-\left(x \leftrightarrow x^{\prime}\right)\right) \\
& -4\left\{H_{a}^{G}(x), H_{b}^{G}\left(x^{\prime}\right)\right\} H^{G a}(x) H^{G b}\left(x^{\prime}\right) .
\end{aligned}
$$

Now smear Eq. (E.3) by two externally prescribed scalar densities of weight $-1, M(x)$ and $N\left(x^{\prime}\right)$. With the abbreviations

$$
M^{a}(x):=M(x) H^{G a}(x), \quad N^{a}(x):=N(x) H^{G a}(x),
$$

Eq. (E.3) becomes 


$$
\{F[M], F[N]\}=\left(2\left\{F[M], H^{G}[\vec{N}]\right\}-(N \leftrightarrow M)\right)-4\left\{H^{G}[\vec{M}], H^{G}[\vec{N}]\right\} .
$$

Note that in Eq. (E.5), $\vec{M}$ and $\vec{N}$ must be treated as externally prescribed smearing vectors. This is because the supermomenta $H^{G a}(x)$, which combined with $M(x)$ and $N(x)$ into the smearing vectors (E.4), stand outside the Poisson brackets in Eq. (E.3).

Since $F(x)$ is a scalar density of weight 2 under Diff $\Sigma$, its Poisson bracket with $H^{G}[\vec{N}]$ is given by

$$
\begin{aligned}
\left\{F[M], H^{G}[\vec{N}]\right\} & =\int_{\Sigma} d^{3} x M(x) £_{\vec{N}} F(x) \\
& =\int_{\Sigma} d^{3} x M\left(F_{, a} N^{a}+2 F N^{a}{ }_{, a}\right) .
\end{aligned}
$$

Therefore the first term on the right-hand side of Eq. (E.5) yields

$$
2\left\{F[M], H^{G}[\vec{N}]\right\}-(N \leftrightarrow M)=4 \int_{\Sigma} d^{3} x F\left(M N_{, a}-N M_{, a}\right) H^{G a} .
$$

The second term on the right-hand side of Eq. (E.5) can be evaluated from the smeared form of Eq. (4.3), which asserts that $H^{G}[\vec{M}]$ represent the Lie algebra Diff $\Sigma$ :

$$
\left\{H^{G}[\vec{M}], H^{G}[\vec{N}]\right\}=H^{G}[[\vec{M}, \vec{N}]] .
$$

Here, $[\vec{M}, \vec{N}]$ is the commutator of the vector fields $\vec{M}$ and $\vec{N}$,

$$
\begin{aligned}
{[\vec{M}, \vec{N}]^{b} } & =N^{b}{ }_{a} M^{a}-M^{b}{ }_{a} N^{a} \\
& =\left(M N_{, a}-N M_{, a}\right) H^{G a} H^{G b} .
\end{aligned}
$$

Equations (E.8) and (E.9) imply that the second term on the right-hand side of Eq. (E.5) exactly compensates the first term given by the integral (E.7). This proves Eq. (E.2).

The Poisson bracket that interests us is

$$
\begin{aligned}
\left\{G(x), G\left(x^{\prime}\right)\right\}= & \left\{F(x), F\left(x^{\prime}\right)\right\} \\
& -\left(2 H_{\perp}^{G}(x)\left\{H_{\perp}^{G}(x), F\left(x^{\prime}\right)\right\}-\left(x \leftrightarrow x^{\prime}\right)\right) \\
& +4 H_{\perp}^{G}(x) H_{\perp}^{G}\left(x^{\prime}\right)\left\{H_{\perp}^{G}(x), H_{\perp}^{G}\left(x^{\prime}\right)\right\} .
\end{aligned}
$$

We have just proved that the first term on the right-hand side of Eq. (E.10) vanishes. Because $H_{\perp}^{G}(x)$ induces an ultralocal change of the metric,

$$
\left\{g^{a b}(x), H_{\perp}^{G}\left(x^{\prime}\right)\right\}=2 K^{a b}(x) \delta\left(x, x^{\prime}\right)
$$

(the coefficient $K^{a b}$ is the extrinsic curvature), the action of $H_{\perp}^{G}$ on $g^{a b}$ in $F$ cancels out under the interchange $x \leftrightarrow x^{\prime}$. Therefore, by Eq. (4.2), the second term on the right-hand side of Eq. (E.10) yields

$$
\begin{aligned}
& -4 H_{\perp}^{G}(x)\left\{H_{\perp}^{G}(x), H_{a}^{G}\left(x^{\prime}\right)\right\} H^{G a}\left(x^{\prime}\right)-\left(x \leftrightarrow x^{\prime}\right) \\
& =4 H_{\perp}^{G}(x) H_{\perp}^{G}\left(x^{\prime}\right) H^{G a}\left(x^{\prime}\right) \delta_{, a^{\prime}}\left(x, x^{\prime}\right)-\left(x \leftrightarrow x^{\prime}\right) .
\end{aligned}
$$

Up to a sign, this is what the last line of Eq. (E.10) yields when we substitute into it the Poisson bracket (4.1). As a result, the right-hand side of Eq. (E.10) vanishes. We have thereby checked the pivotal equation (4.22) of the main text. 


\section{APPENDIX F: DiffS AND THE EIGENFUNCTIONALS OF $\hat{G}(z)$}

We want to prove that if $\boldsymbol{\phi}[\boldsymbol{g}]$ is an eigenfunctional of $\hat{\boldsymbol{G}}(z)$ with eigenvalue $\boldsymbol{G}(z)$,

$$
\hat{\boldsymbol{G}}(z) \boldsymbol{\phi}[\boldsymbol{g}]=\boldsymbol{G}(z) \boldsymbol{\phi}[\boldsymbol{g}]
$$

then $\boldsymbol{\phi}\left[\boldsymbol{\Xi}^{*} \boldsymbol{g}\right]$ is an eigenfunctional of $\hat{\boldsymbol{G}}(z)$ with eigenvalue $\left(\boldsymbol{\Xi}^{-1}\right)^{*} \boldsymbol{G}(z)$ :

$$
\hat{\boldsymbol{G}}(z) \boldsymbol{\phi}\left[\boldsymbol{\Xi}^{*} \boldsymbol{g}\right]=\left(\boldsymbol{\Xi}^{-1}\right)^{*} \boldsymbol{G}(z) \boldsymbol{\phi}\left[\boldsymbol{\Xi}^{*} \boldsymbol{g}\right]
$$

It is sufficient to prove the infinitesimal version of Eq. (F.2), namely

$$
\left.\frac{d}{d \sigma}\left(\hat{\boldsymbol{G}}(z) \boldsymbol{\phi}\left[\boldsymbol{\Xi}_{\sigma}^{*} \boldsymbol{g}\right]\right)\right|_{\sigma=0}=\left.\frac{d}{d \sigma}\left(\left(\boldsymbol{\Xi}_{\sigma}^{-1}\right)^{*} \boldsymbol{G}(z) \boldsymbol{\phi}\left[\boldsymbol{\Xi}_{\sigma}^{*} \boldsymbol{g}\right]\right)\right|_{\sigma=0}
$$

and then to pass to an arbitrary finite diffeomorphism $\boldsymbol{\Xi}$ by exponentiation in $\sigma$.

To begin, recall that the classical charge $Q[\overrightarrow{\boldsymbol{\xi}}]$ discussed in Sec. 5b is the canonical generator of Diff $\mathcal{S}$. Thus, the action of Diff $\mathcal{S}$ on the operator $\hat{\boldsymbol{G}}(z)$ is given by the commutator

$$
\frac{1}{i}[\hat{\boldsymbol{G}}(z), \hat{Q}[\overrightarrow{\boldsymbol{\xi}}]]=£_{\overrightarrow{\boldsymbol{\xi}}} \hat{\boldsymbol{G}}(z)
$$

Also observe that the change in $\boldsymbol{\phi}[\boldsymbol{g}]$ under an infinitesimal diffeomorphism of its argument $\boldsymbol{g}$ is determined by the operator $\hat{Q}[\overrightarrow{\boldsymbol{\xi}}]$ acting on $\boldsymbol{\phi}[\boldsymbol{g}]$ :

$$
\begin{aligned}
\left.\frac{d}{d \sigma} \boldsymbol{\phi}\left[\boldsymbol{\Xi}_{\sigma}^{*} \boldsymbol{g}\right]\right|_{\sigma=0} & =\int_{\mathcal{S}} d^{3} z\left(£_{\overrightarrow{\boldsymbol{\xi}}} \boldsymbol{g}_{k \ell}(z)\right) \frac{\delta \boldsymbol{\phi}[\boldsymbol{g}]}{\delta \boldsymbol{g}_{k \ell}(z)} \\
& =i \int_{\mathcal{S}} d^{3} z\left(£_{\overrightarrow{\boldsymbol{\xi}}} \hat{\boldsymbol{g}}_{k \ell}(z)\right) \hat{\boldsymbol{p}}^{k \ell}(z) \boldsymbol{\phi}[\boldsymbol{g}] \\
& =i \hat{Q}[\overrightarrow{\boldsymbol{\xi}}] \boldsymbol{\phi}[\boldsymbol{g}] .
\end{aligned}
$$

By letting $\hat{\boldsymbol{G}}(z)$ act on equation (F.5) and using the commutator (F.4), we obtain

$$
\begin{aligned}
\left.\frac{d}{d \sigma}\left(\hat{\boldsymbol{G}}(z) \boldsymbol{\phi}\left[\boldsymbol{\Xi}_{\sigma}^{*} \boldsymbol{g}\right]\right)\right|_{\sigma=0} & =i[\hat{\boldsymbol{G}}(z), \hat{Q}[\overrightarrow{\boldsymbol{\xi}}]] \boldsymbol{\phi}[\boldsymbol{g}]+i \hat{Q}[\overrightarrow{\boldsymbol{\xi}}] \hat{\boldsymbol{G}}(z) \boldsymbol{\phi}[\boldsymbol{g}] \\
& =-\left(£_{\overrightarrow{\boldsymbol{\xi}}} \hat{\boldsymbol{G}}(z)\right) \boldsymbol{\phi}[\boldsymbol{g}]+i \hat{Q}[\overrightarrow{\boldsymbol{\xi}}] \hat{\boldsymbol{G}}(z) \boldsymbol{\phi}[\boldsymbol{g}]
\end{aligned}
$$

Now use Eq. (F.5) along with the fact that $\boldsymbol{\phi}[\boldsymbol{g}]$ is an eigenfunctional of $\hat{\boldsymbol{G}}(z)$. Equation (F.6) then becomes

$$
\left.\frac{d}{d \sigma}\left(\hat{\boldsymbol{G}}(z) \boldsymbol{\phi}\left[\boldsymbol{\Xi}_{\sigma}^{*} \boldsymbol{g}\right]\right)\right|_{\sigma=0}=\left.\frac{d}{d \sigma}\left(\left(\boldsymbol{\Xi}_{\sigma}^{-1}\right)^{*} \boldsymbol{G}(z)\right)\right|_{\sigma=0} \boldsymbol{\phi}[\boldsymbol{g}]+\left.\boldsymbol{G}(z) \frac{d}{d \sigma}\left(\boldsymbol{\phi}\left[\boldsymbol{\Xi}_{\sigma}^{*} \boldsymbol{g}\right]\right)\right|_{\sigma=0}
$$

which is equivalent to Eq. (F.3). This proves the theorem expressed in Eq. (F.2). 


\section{APPENDIX G: RELATIVISTIC PARTICLE IN THE PROPER TIME FORMALISM}

As in Appendix D, let us regard the rest mass $m$ for the relativistic particle as a dynamical variable canonically conjugate to the proper time $\tau$ along the particle's worldline:

$$
\{\tau, m\}=1 \text {. }
$$

The proper time formalism for the relativistic particle is obtained by resolving the superHamiltonian constraint in the extended phase space $\left(\tau, y^{\alpha} ; m, P_{\alpha}\right)$,

$$
H_{\mathrm{ext}}:=m^{2}+\gamma^{\alpha \beta}(y) P_{\alpha} P_{\beta}=0,
$$

with respect to $m$. This yields

$$
h_{\uparrow}:=m+h=0, \quad h:=-\sqrt{-\gamma^{\alpha \beta}(y) P_{\alpha} P_{\beta}},
$$

where the choice of the negative square root in the Hamiltonian $h$ ensures the positivity of $m$. The reality of $m$ requires that

$$
G:=-\gamma^{\alpha \beta}(y) P_{\alpha} P_{\beta}>0
$$

i.e., that the particle moves along a timelike worldline.

The imposition of the constraint (G.3) as an operator restriction on the state function $\Psi\left(\tau, y^{\alpha}\right)$ yields the Schrödinger equation in proper time $\tau$ :

$$
\hat{H}_{\uparrow} \Psi=0 \quad \Longleftrightarrow \quad i \partial_{\tau} \Psi(\tau, y)=\hat{h} \Psi(\tau, y) .
$$

The Hamiltonian

$$
\hat{h}=-\sqrt{-\hat{G}}, \quad \hat{G}=\square
$$

must be defined by spectral analysis. It is self-adjoint on the Hilbert space $\mathcal{H}^{+}$spanned by the eigenfunctions $\phi_{G}$ of $\hat{G}$ belonging to positive eigenvalues $G$ :

$$
\hat{G} \phi_{G}(y)=G \phi_{G}(y), \quad G>0 \text {. }
$$

On $\mathcal{H}^{+}$, the Schrödinger inner product

$$
\langle\Psi \mid \Psi\rangle:=\int d^{4} y \bar{\Psi}(\tau, y) \Psi(\tau, y)
$$

is conserved in $\tau$. Observables must leave the states in $\mathcal{H}^{+}$, i.e., they must satisfy the condition (6.46), where

$$
\hat{\mathcal{P}}^{+}=\Theta(\hat{G})
$$

is the projector to $\mathcal{H}^{+}$. 
In general, neither $\hat{y}^{\alpha}=y^{\alpha} \times$ nor $\hat{P}_{\alpha}=-i \partial / \partial y^{\alpha}$ are observables. This prevents us from interpreting the multiplication operator $\hat{y}^{\alpha}$ as an observable whose measurement at the proper time $\tau$ would localize the particle at a spacetime event $y^{\alpha}$, and $\bar{\Psi}(\tau, y) \Psi(\tau, y)$ as the probability density that the localization will occur at $y^{\alpha}$.

This difficulty persists even for a particle moving in flat spacetime $\gamma^{\alpha \beta}(y)=\eta^{\alpha \beta}$. In this case, $\hat{P}_{\alpha}$ commutes with $\hat{G}$ and hence it is an observable. On the other hand, one can explicitly show that $\hat{y}^{\alpha}=y^{\alpha} \times$ is not an observable since one can always choose a constant covector $\pi_{\alpha}$ such that the action of the unitary operator

$$
\hat{U}_{\pi}:=\exp \left(i \pi_{\alpha} \hat{y}^{\alpha}\right)
$$

leads the state $\Psi$ out of $\mathcal{H}^{+}$.

The demonstration follows the general outline of an old argument by Pauli [28] that if time were represented by the multiplication operator, the energy spectrum could not be bounded from below. In our example, the Hilbert space $\mathcal{H}$ is spanned by the joint eigenfunctions

$$
\Phi_{P}(y)=(2 \pi)^{-2} \exp \left(i P_{\alpha} y^{\alpha}\right)
$$

of the momentum operators $\hat{P}_{\alpha}$. Equation

$$
\Psi(y)=\int d^{4} P \tilde{\Psi}(P) \Phi_{P}(y)
$$

relates the $y$-representation $\Psi\left(y^{\alpha}\right)$ of a state $\Psi \in \mathcal{H}$ to its $P$-representation $\tilde{\Psi}\left(P_{\alpha}\right)$. The momentum eigenstates (G.11) are also eigenstates of $\hat{G}$ with eigenvalues $G=-\eta^{\alpha \beta} P_{\alpha} P_{\beta}$. The state $\Psi$ lies in $\mathcal{H}^{+}$if the state function $\tilde{\Psi}\left(P_{\alpha}\right)$ has its support only on timelike vectors $P_{\alpha}:$

$$
\tilde{\Psi}\left(P_{\alpha}\right)=0 \text { for } \eta^{\alpha \beta} P_{\alpha} P_{\beta} \geq 0
$$

The action of $\hat{U}_{\pi}$ on the state function $\tilde{\Psi}\left(P_{\alpha}\right)$ translates its argument by $-\pi_{\alpha}$ :

$$
\left(\hat{U}_{\pi} \tilde{\Psi}\right)\left(P_{\alpha}\right)=\tilde{\Psi}\left(P_{\alpha}-\pi_{\alpha}\right)
$$

The support of $\left(\hat{U}_{\pi} \tilde{\Psi}\right)\left(P_{\alpha}\right)$ is thereby shifted by the amount $\pi_{\alpha}$ relative to the support of $\tilde{\Psi}\left(P_{\alpha}\right)$. Because $\pi_{\alpha}$ is arbitrary, the support of $\left(\hat{U}_{\pi} \tilde{\Psi}\right)\left(P_{\alpha}\right)$ cannot always remain timelike. This means that $\hat{y}^{\alpha}$ is not an observable.

This simple example illustrates why the fundamental gravitational variables cannot be expected to be observables. 


\section{REFERENCES}

[1] J. A. Wheeler, in Batelle Rencontres: 1967 Lectures in Mathematics and Physics, edited by C. DeWitt and J. A. Wheeler (Benjamin, New York, 1968).

[2] B. S. DeWitt, Phys. Rev. 160, 1113 (1967).

[3] K. V. Kuchař, in Quantum Gravity 2: A Second Oxford Symposium, edited by C. J. Isham, R. Penrose, and D. W. Sciama (Clarendon, Oxford, 1981).

[4] K. V. Kuchař, J. Math. Phys. 22, 2640 (1981).

[5] K. V. Kuchař, in Proceedings of the 4th Canadian Conference on General Relativity and Relativistic Astrophysics, edited by G. Kunstatter, D. Vincent, and J. Williams (World Scientific, Singapore, 1992).

[6] A. Einstein, Über die Spezielle und die Allgemeine Relativitätstheorie (Vieweg, Braunschweig, 1920); English translation by R. W. Lawson, Relativity: The Special and the General Theory (Crown, New York, 1961).

[7] D. Hilbert, 2 Mitt., Nachr. Ges. Wiss. Göttingen 53 (1917).

[8] C. Møller, The Theory of Relativity (Oxford University Press, Oxford, 1952).

[9] L. D. Landau and E. M. Lifshitz, The Classical Theory of Fields, 4th edition (Pergamon Press, Oxford, 1989).

[10] K. V. Kuchař and C. G. Torre, Phys. Rev. D 43, 419 (1991).

[11] K. V. Kuchař and C. G. Torre, Phys. Rev. D 44, 3116 (1991).

[12] C. L. Stone and K. V. Kuchař, Class. Quantum Grav. 9, 757 (1991).

[13] K. V. Kuchař, Phys. Rev. D 44, 43 (1992).

[14] B. S. DeWitt, in Gravitation: An Introduction to Current Research, edited by L. Witten (Wiley, New York,1962).

[15] J. D. Brown, "On variational principles for gravitating perfect fluids", submitted to Class. Quantum Grav.

[16] Our dust action is based on the canonical relativistic perfect fluid action discussed in J. D. Brown, Class. Quantum Grav. 10, 1579 (1993). This action makes use of a velocity-potential or "Clebsch" representation of the fluid velocity (A. Clebsch, J. Reine Angew. Math. 56, 1 (1859)). Clebsch potentials were used in the development of action principles for nonrelativistic fluids by Lin (C. C. Lin, in Liquid Helium, edited by G. Careri (Academic Press, New York, 1963); see also J. Serrin, in Handbuch der Physik, volume 8, edited by S. Flügge and C. Truesdell (Springer, Berlin, 1959)) and by Seliger and Whitham (R. L. Seliger and G. B. Whitham, Proc. Roy. Soc. A 305, 1 (1968)). Tam used Clebsch potentials to formulate an action principle for an ideal, charged fluid in the context of special relativity (K. Tam, Can. J. Phys. 44, 2403 (1966)). Action principles for perfect fluids in general relativity were developed independently by Tam and O'Hanlon (K. Tam and J. O'Hanlon, Nuovo Cimento 62B, 351 (1969)) and by Schutz (B. F. Schutz, Phys. Rev. D 2, 2762 (1970). The actions discussed by Tam and O'Hanlon and by Schutz use a minimal set of velocity potentials, which precludes the interpretation of the appropriate potential fields as Lagrangian coordinates for the fluid. The Hamiltonian form of Schutz's action was developed by Schutz (B. F. Schutz, Phys. Rev. D 4, 3559 (1971)) and by Demaret and Moncrief (J. Demaret and V. Moncrief, Phys. Rev. D 21, 2785 (1980)).

[17] K. V. Kuchař, in Directions in General Relativity, volume 1, edited by B. L. Hu, M. P. 
Ryan, and C. V. Vishveshwara (Cambridge University Press, Cambridge, 1993); J. D. Brown, in Proceedings of the Cornelius Lanczos Centenary Conference, edited by J. D. Brown, M. T. Chu, D. C. Ellison, and R. J. Plemmons (SIAM, Philadelphia, in press).

[18] C. Carathèodory, Variationsrechnung und Partielle Differentialgleichungen Erster Ordnung I (Teubner, Leipzig, 1956).

[19] M. Born, Proc. Roy. Soc. A. 143, 410 (1934); M. Born and L. Infeld, Proc. Roy. Soc. A. 144, 425 (1934).

[20] C. J. Isham and K. V. Kuchař, Ann. Phys. (N.Y.) 164, 288 and 316 (1985).

[21] S. Hojman, K. V. Kuchař and C. Teitelboim, Ann. Phys. (N.Y.) 96, 88 (1974).

[22] J. L. Synge, Relativity: The General Theory (North Holland, Amsterdam, 1964).

[23] K. V. Kuchař, Phys. Rev. D 39, 1579 (1989).

[24] A. Hanson, T. Regge, and C. Teitelboim, Constrained Hamiltonian Systems (Accad. Naz. dei Lincei, Rome, 1976); L. Brink and M. Henneaux, Principles of String Theory (Plenum, New York, 1988); K. V. Kuchař and C. G. Torre, J. Math. Phys. 30, 1769 (1989).

[25] C. Teitelboim, in Quantum Theory of Gravity, edited by S. M. Christensen (Adam Hilger, Bristol, 1984); C. G. Torre, Phys. Rev. D 40, 2588 (1989).

[26] J. D. Brown, Ref. [15].

[27] B. F. Schutz, first citation in Ref. [15].

[28] W. Pauli, in Die Allgemeinen Prinzipien der Wellenmechanik, Handbuch der Physik V.1, edited by S. Flügge (Springer, Berlin, 1958). 


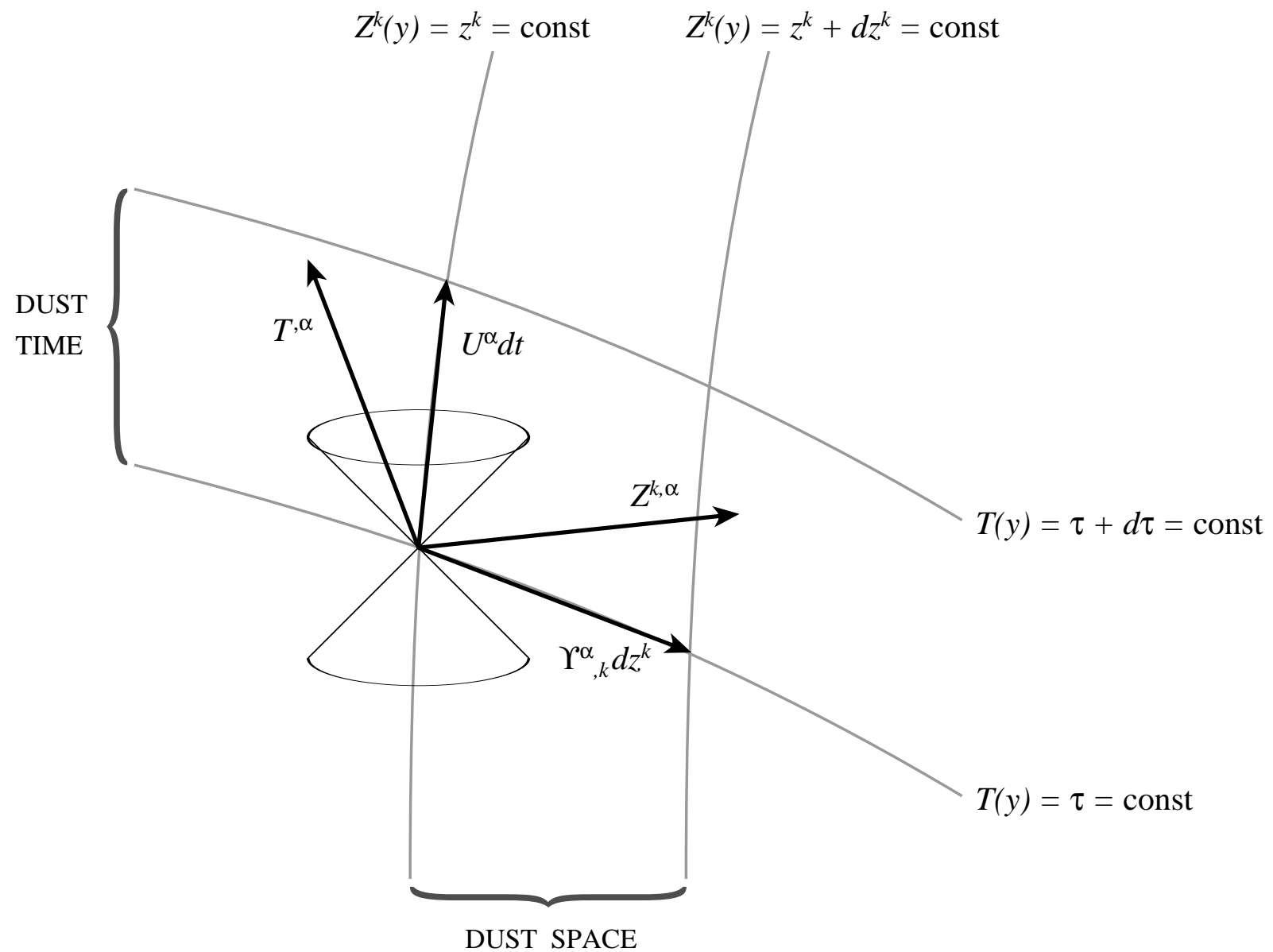

Figure 1: $U^{\alpha} \partial / \partial y^{\alpha}=\partial / \partial \tau$ and $\Upsilon^{\alpha}{ }_{, k} \partial / \partial y^{\alpha}=\partial / \partial z^{k}$ are the coordinate basis vectors for the dust coordinates $\left(\tau, z^{k}\right)$. The covectors $T_{, \alpha} d y^{\alpha}=d \tau$ and $Z^{k}{ }_{, \alpha} d y^{\alpha}=d z^{k}$ correspond to the stacks of surfaces $\tau=$ const and $z^{k}=$ const, respectively; the associated vectors are $T^{, \alpha}:=g^{\alpha \beta} T_{, \beta}$ and $Z^{k, \alpha}:=g^{\alpha \beta} Z^{k}{ }_{, \beta}$. The vectors $U^{\alpha}$ and $Z^{k, \alpha}$ are orthogonal, $U^{\alpha} Z^{k}{ }_{, \alpha}=0$, and are therefore drawn symmetrically about the light cone. Likewise, the vectors $T^{, \alpha}$ and $\Upsilon_{, k}^{\alpha}$ are orthogonal, $T_{, \alpha} \Upsilon^{\alpha}{ }_{, k}=0$, and are drawn symmetrically about the light cone. 


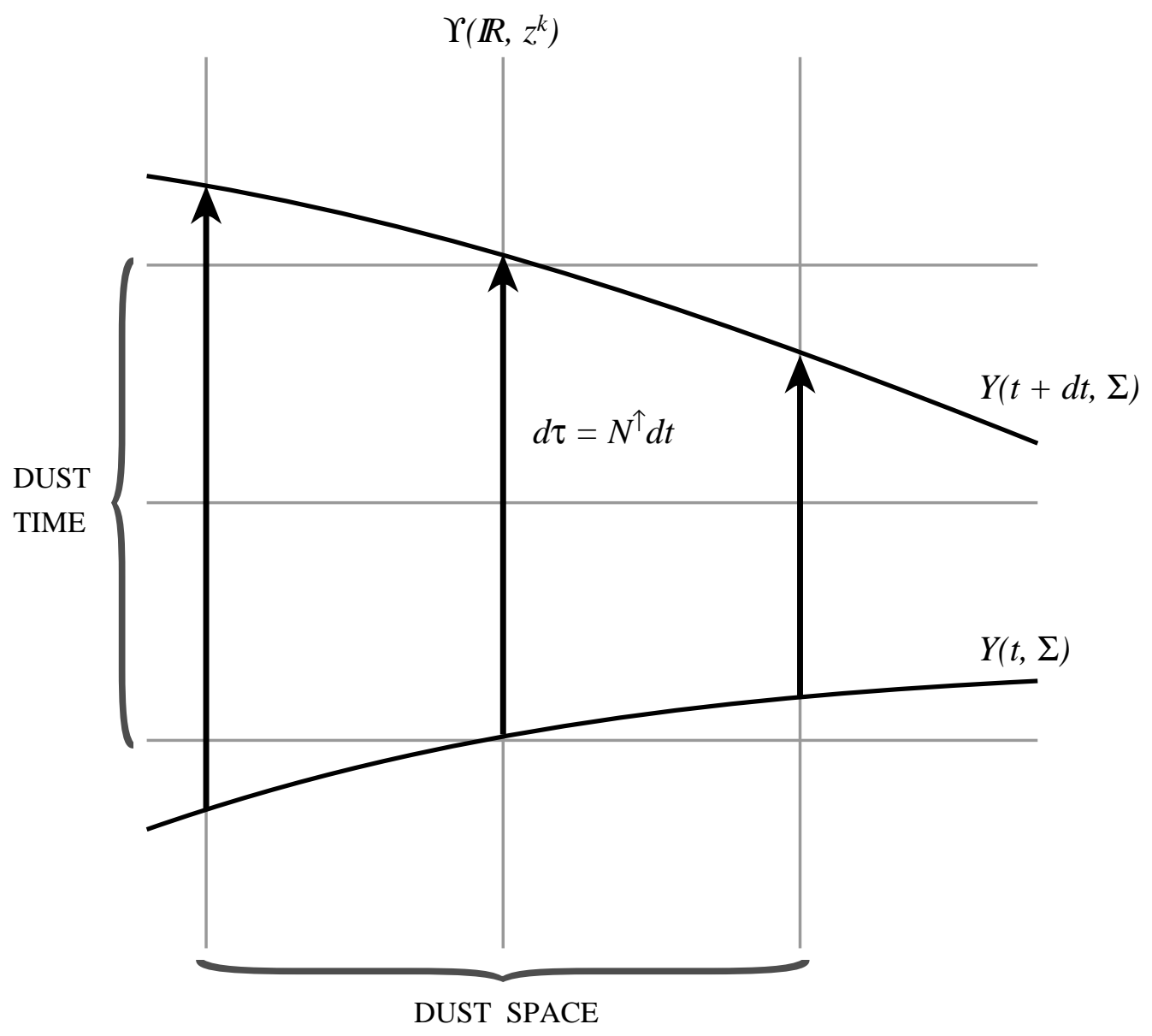

Figure 2: The hypersurface $Y(t, \Sigma)$ is displaced to the hypersurface $Y(t+d t, \Sigma)$ along the dust flow lines $z^{k}=$ const by the proper time $d \tau=N^{\uparrow}\left(x=Z^{-1}(z)\right) d t$. The displacement is generated by the Poisson brackets with $H\left[N^{\uparrow}\right] d t$. 


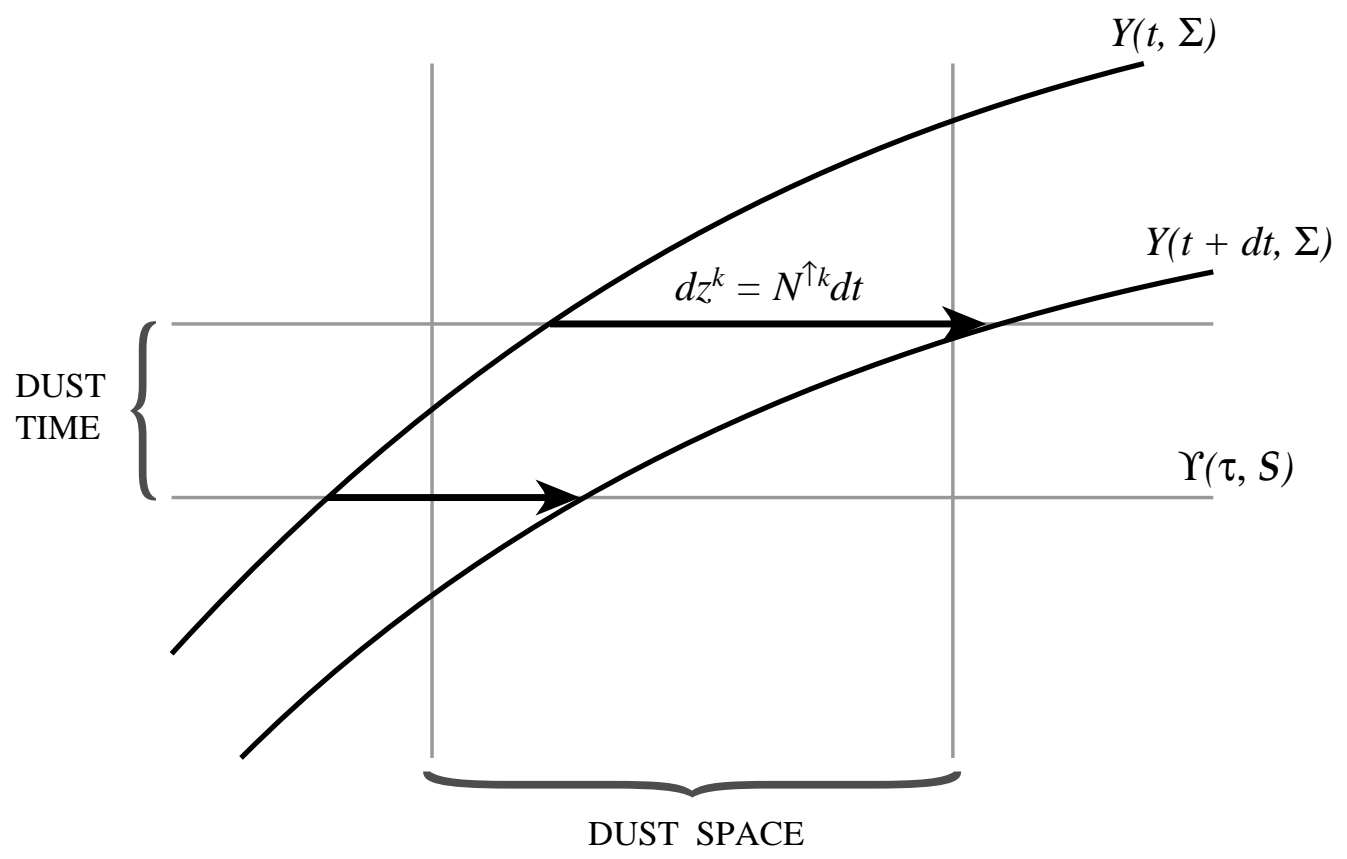

Figure 3: The hypersurface $Y(t, \Sigma)$ is displaced to the hypersurface $Y(t+d t, \Sigma)$ along the leaves $\Upsilon(\tau, \mathcal{S})$ of the dust time foliation $\mathcal{T}$ by the amounts $d z^{k}=N^{\uparrow k}\left(x=Z^{-1}(z)\right) d t$ in the dust space $\mathcal{S}$. The displacement is generated by the Poisson brackets with $H\left[\vec{N}^{\uparrow}\right] d t$. 